\title{
Localized radial solutions of the Swift-Hohenberg equation
}

\author{
David Lloyd \\ Department of Mathematics \\ University of Surrey \\ Guildford, GU2 7XH \\ United Kingdom
}

\author{
Björn Sandstede* \\ Department of Mathematics \\ University of Surrey \\ Guildford, GU2 7XH \\ United Kingdom
}

December 3, 2008

\begin{abstract}
Stationary localized solutions of the planar Swift-Hohenberg equation are investigated in the parameter region where the trivial solution is stable. In the parameter region where rolls bifurcate subcritically, localized radial ring-like pulses are shown to bifurcate from the trivial solution. Furthermore, radial spot-like pulses are shown to bifurcate from the trivial state, regardless of the criticality of roll patterns. These theoretical results apply also to general reaction-diffusion systems near Turing instabilities. Numerical computations show that planar radial pulses 'snake' near the Maxwell point where, by definition, the one-dimensional roll patterns have the same energy as the trivial state. These computations also reveal that spots, which are stable in a certain parameter region, become unstable with respect to hexagonal perturbations, leading to fully localized hexagon patterns.
\end{abstract}

\section{Introduction}

In this paper, we investigate the existence of localized stationary radial solutions of the Swift-Hohenberg equation

$$
u_{t}=-(1+\Delta)^{2} u-\mu u+f(u), \quad(x, y) \in \mathbb{R}^{2},
$$

where

$$
f(u)=\nu u^{2}-\kappa u^{3}+\mathrm{O}\left(u^{4}\right) .
$$

Stationary radial solutions $u(r)$ depend only on the radial variable $r=\sqrt{x^{2}+y^{2}}$ and therefore satisfy the ordinary differential equation

$$
\left(1+\Delta_{r}\right)^{2} u=-\mu u+f(u),
$$

where $\Delta_{r}=\partial_{r}^{2}+\frac{1}{r} \partial_{r}$. We are interested in finding localized solutions $u(r)$ of (1.2) that decay to zero as $r \rightarrow \infty$ and shall therefore seek such solutions for $\mu>0$, where the background state $u=0$ is stable. More restrictively, our results are valid only for $0<\mu \ll 1$ : the trivial state $u=0$ destabilizes at $\mu=0$, and we shall construct localized radial solutions with small amplitude that bifurcate from $u=0$ at $\mu=0$ into the region $\mu>0$.

Before stating our results, we outline briefly our interest in the Swift-Hohenberg equation and its localized radial steady states. The Swift-Hohenberg equation is a general model for pattern-forming processes which was first derived by Swift and Hohenberg [39] to describe random thermal fluctuations in the Boussinesq equation. Equation (1.1) exhibits many interesting localized and non-localized patterns, and we refer to $[10,14,17,29,30]$ for details; see also Figure 1 and $\S 9$. In particular, localized radial pulses and localized hexagon and rhomboid patches have been found

*Current address: Division of Applied Mathematics, Brown University, Providence, RI 02912, USA 


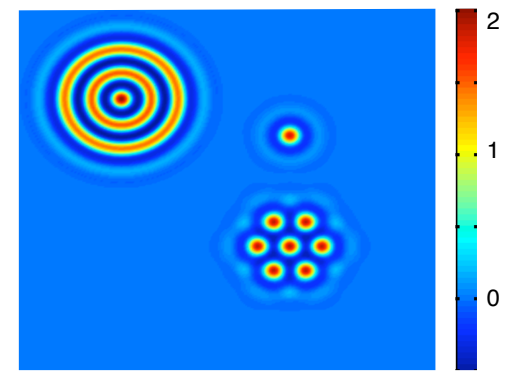

Figure 1: Spatially localized stationary spots and hexagon patches of (1.1) with $f(u)=\nu u^{2}-u^{3}$ at $(\mu, \nu)=(0.5,2.2)$.
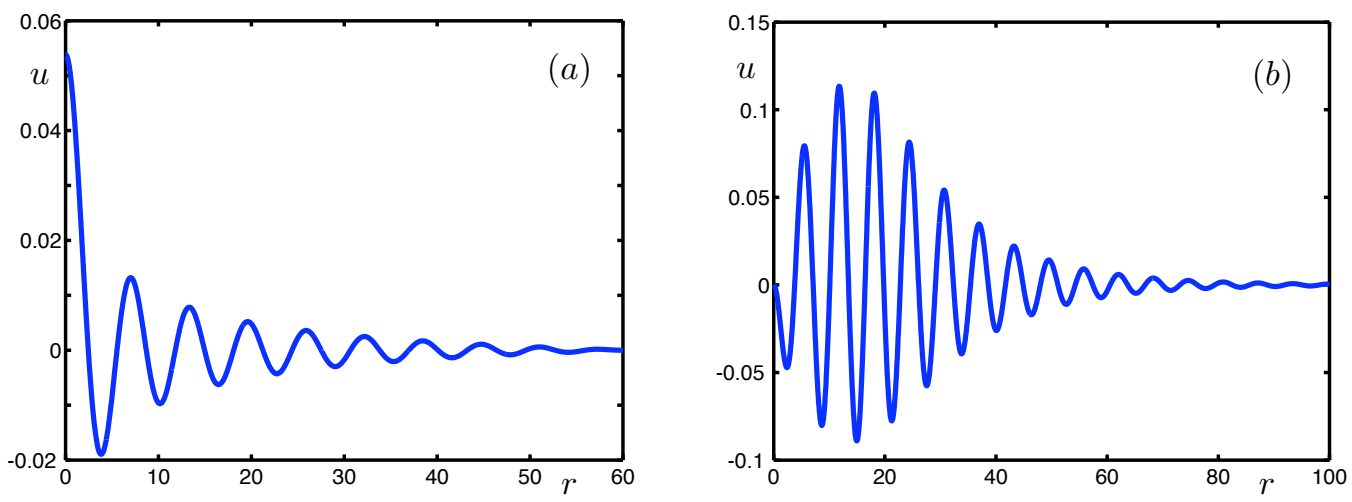

Figure 2: Stationary radial profiles of (1.1) with $f(u)=\nu u^{2}-u^{3}$ : (a) spots for $(\mu, \nu)=(0.005,1.833)$; (b) rings for $(\mu, \nu)=(0.014,2.231)$

in numerical investigations of the Swift-Hohenberg equation. It was observed in [24] that localized hexagon patches can bifurcate from radial pulses via symmetry-breaking bifurcations. It is this observation that motivated us to find out more about the existence of radial pulses.

Numerically, we found two types of radial pulses in the Swift-Hohenberg equation: spots, which resemble the Bessel function $J_{0}(r)$, see Figure 2(a), and rings that have a pulse-shaped envelope in the far field as indicated in Figure 2(b). Analytically, we can prove the existence of radial pulses with small amplitude near the Turing instability of the trivial state $u=0$, which occurs for $\mu=0$.

Theorem 1 (Existence of rings) Fix $(\nu, \kappa)$ so that

$$
c_{3}^{0}:=\frac{3 \kappa}{4}-\frac{19 \nu^{2}}{18}<0 .
$$

For each integer $n \geq 0$, there is a $\mu_{*}>0$ such that the Swift-Hohenberg equation (1.1) has two stationary localized radial ring solutions $u(r)$ for each $\mu \in\left(0, \mu_{*}\right)$ with the following properties: we have $u(r)=\mathrm{O}\left(\mu^{\frac{3}{4}}\right)$ as $\mu \rightarrow 0$ on each fixed interval $\left[0, r_{*}\right]$, and there is $a \varphi \in \mathbb{R}$ and a function $q_{n}(s)$ that has precisely $n$ simple zeros such that $u(r) \approx \pm \sqrt{\mu} q_{n}(\sqrt{\mu} r) \cos (r+\varphi)$ for large $r$. Ring solutions with these properties do not bifurcate when $c_{3}^{0}>0$.

We shall comment below on the interpretation of the number $c_{3}^{0}$ that appears in Theorem 1 ; the envelopes $q_{n}(s)$ are discussed further in Lemma 4, and a more detailed asymptotic expansion of rings near $r=0$ can be found in (4.36). In contrast to rings, which bifurcate only when $c_{3}^{0}<0$, spots bifurcate for any fixed nonzero value of $\nu$.

Theorem 2 (Existence of spots for $\nu \neq 0$ ) Fix $\nu \neq 0$ and any $\kappa \in \mathbb{R}$, then there is a $\mu_{*}>0$ such that the Swift-Hohenberg equation (1.1) has a stationary localized radial solution $u(r)$ for each $\mu \in\left(0, \mu_{*}\right)$ : these solutions stay close to $u=0$ and, for each fixed $r_{*}>0$, we have the asymptotics $u(r)=\alpha \sqrt{\mu} J_{0}(r)+\mathrm{O}(\mu)$ as $\mu \rightarrow 0$ uniformly in $0 \leq r \leq r_{*}$ for an appropriate constant $\alpha$ with $\operatorname{sign} \alpha=\operatorname{sign} \nu$. 


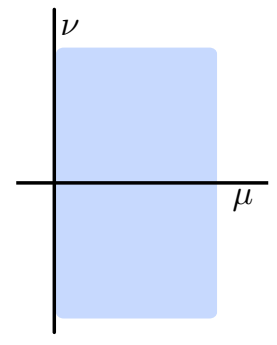

rings for $\kappa<0$

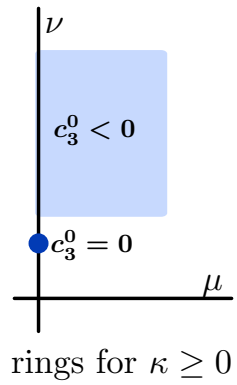

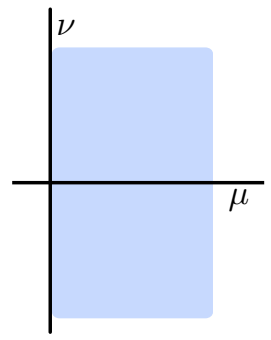

spots for $\kappa<0$

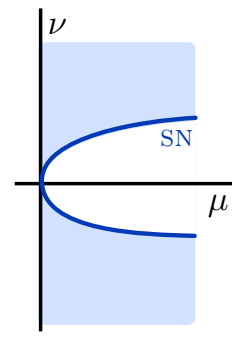

spots for $\kappa>0$

Figure 3: The shaded regions correspond to the existence regions of rings and spots that are given in Theorems 1-3 for the nonlinearity $f(u)=\nu u^{2}-\kappa u^{3}$. Spots undergo fold bifurcations along the curve labelled SN; see Theorem 3(ii).

The following result covers the existence of spots for $\nu$ equal to or close to zero.

\section{Theorem 3 (Existence of spots for $\nu \approx 0$ )}

(i) If $\nu=0$ and $\kappa<0$ is fixed, then small-amplitude spots bifurcate from $u=0$ at $\mu=0$ into the region $\mu>0$, and their amplitude $u(0)$ scales with $\mu^{\frac{1}{4}}|\log \mu|^{-\frac{1}{2}}$.

(ii) If $\nu=0$ and $\kappa>0$ is fixed, spots do not bifurcate from $u=0$ : instead, for $(\mu, \nu)$ close to zero, spots undergo a fold bifurcation along the curve $\nu= \pm \mu^{\frac{1}{4}}|\log \mu|^{\frac{1}{2}}[c+\mathrm{o}(1)]$, where $0<\mu \ll 1$ and $c>0$ is a constant, and their amplitude $u(0)$ scales with $\mu^{\frac{1}{4}}|\log \mu|^{-\frac{1}{2}}$.

Spots and rings are initially unstable with respect to the PDE dynamics of the Swift-Hohenberg equation (1.1). However, our numerical continuation results indicate that spots and rings stabilize in a certain region in the $(\mu, \nu)$ parameter space, at least when the nonlinearities $f(u)=\nu u^{2}-u^{3}$ or $f(u)=\kappa u^{3}-u^{5}$ are used. Our computations also show that both spots and rings exhibit snaking, which we shall discuss in detail in $\S 8$.

Remark 1 Though our results are stated only for the Swift-Hohenberg equation, they apply to general reactiondiffusion systems

$$
U_{t}=D \Delta U+F(U, \mu), \quad(x, y) \in \mathbb{R}^{2}, \quad U \in \mathbb{R}^{N}
$$

near Turing instabilities: indeed, it was shown in [36, Chapter 3] that localized radial patterns of small amplitude are captured by a four-dimensional nonautonomous differential equation in the radius $r$ which coincides, to leading order, with the radial steady-state equation of the Swift-Hohenberg equation that we study here.

We now comment in more detail on the results stated above. Theorem 1 is not a surprising result. As mentioned above, the trivial state $u=0$ undergoes a Turing instability at $\mu=0$. In the far field, for $r \gg 1$, we therefore expect small-amplitude roll patterns to bifurcate. It turns out that these patterns bifurcate supercritically into the region $\mu<0$ for $c_{3}^{0}>0$ and subcritically into the region $\mu>0$ when $c_{3}^{0}<0$. Thus, we can expect to find radial pulses that resemble rolls in an intermediate spatial region before decaying to zero as $r \rightarrow \infty$ only when $c_{3}^{0}<0$, where rolls actually exist. This is what Theorem 1 asserts, in line with the numerical computations that we shall present in $\S 8$. Note that the ring pulses shown in Figure 2(b) indeed begin with small amplitude near the core and grow towards a roll pattern before converging to zero. The rolls that are found inside ring pulses bifurcate subcritically and are therefore PDE unstable: our numerical computations in $\S 8$ indicate that the bifurcating rings have two unstable PDE eigenvalues near bifurcation. In summary, rings bifurcate in the parameter region where rolls coexist with the PDE stable trivial background state. The situation for spots is very different. Perhaps surprisingly, Theorem 2 states that spots bifurcate for any value of $\nu>0$, regardless of the value of $\kappa$. In particular, there is no condition on the sign of $c_{3}^{0}$, and spots exist even when the far-field equation does not admit any rolls.

While radial pulses have been studied extensively for the nonlinear Schrödinger equation and the complex GinzburgLandau equation, we are not aware of any existence results for radial pulses of the Swift-Hohenberg equation. 

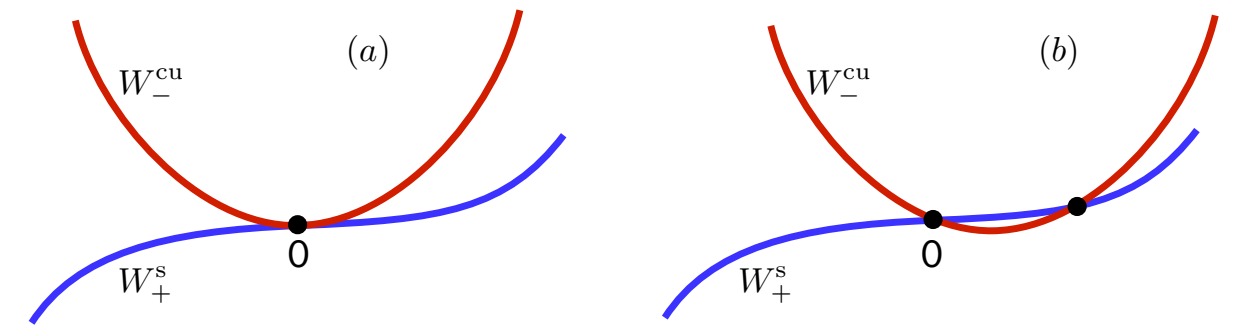

Figure 4: Schematic diagrams of the geometry of the core manifold $W_{-}^{\mathrm{cu}}$ and the stable manifold $W_{+}^{\mathrm{s}}$ are shown in panel (a) for $\mu=0$ and in panel (b) for $0<\mu \ll 1$.

Mountain-pass methods, which have proved useful in proving existence for the nonlinear Schrödinger equation and fourth-order differential equations (see, for instance, $[16,20]$ ), appear to fail here since the Lyapunov functional is bounded from below due to the cubic restabilizing term. Topological shooting methods [28] have been utilized with great success to prove the existence of pulses for the 1D Swift-Hohenberg equation. However, the apparent lack of a Lyapunov functional for the radial equation (1.2) seems to make it difficult to use these methods in our setting.

Our approach is motivated by Scheel's work [36] on the bifurcation to non-localized target patterns near supercritical Turing instabilities in reaction-diffusion systems. We consider (1.2) as the nonautonomous first-order differential equation

$$
\frac{\mathrm{d}}{\mathrm{d} r}\left(\begin{array}{c}
u_{1} \\
u_{2} \\
u_{3} \\
u_{4}
\end{array}\right)=\left(\begin{array}{c}
u_{3} \\
u_{4} \\
-u_{1}-\frac{1}{r} u_{3}+u_{2} \\
-u_{2}-\frac{1}{r} u_{4}-\mu u_{1}+f(u)
\end{array}\right), \quad U=\left(u_{1}, u_{2}, u_{3}, u_{4}\right)^{T}
$$

in the radial evolution variable $r$. Scheel's approach was to derive an $r$-dependent normal form in the far field for $r \gg 1$ which, in the limit as $r \rightarrow \infty$, becomes the standard Ginzburg-Landau equation that describes Turing patterns near onset. Using this information, we show that the $r$-dependent far-field equation possesses localized pulses precisely when $c_{3}^{0}<0$. Due to the employed scaling, we can control the localized pulses initially only in the region $r>r_{1} / \sqrt{\mu}$ for fixed $r_{1}$. On the other hand, regular perturbation theory using Bessel functions allows us to describe all solutions that stay bounded near the core for $0 \leq r \leq r_{0}$ for any fixed $r_{0}$. To match the two sets of solutions at $r=r_{0}$, we employ a different scaling to track the localized far-field pulses from $r=r_{1} / \sqrt{\mu}$ back to $r=r_{0}$. Using this approach, which is similar to the one pursued in [36], we find the ring pulses described in Theorem 1.

The mechanism that gives rise to spots is different. For fixed $\mu>0$, the origin is an asymptotically hyperbolic equilibrium at $r=\infty$ with respect to equation (1.3). Thus, we can use the $r$-dependent far-field equation to describe the tangent space at $u=0$ of the stable manifold of $U=0$, that is, the tangent space at $U=0$ of the set of solutions that stay close to $U=0$ and converge to $U=0$ as $r \rightarrow \infty$. Tracking this two-dimensional tangent space back to $r=r_{0}$ and then setting $\mu=0$, we find that the resulting two-dimensional subspace has a one-dimensional intersection with the tangent space of solutions of (1.3) that stay bounded as $r \rightarrow 0$. In fact, as illustrated in Figure 4, the associated nonlinear manifolds have a nondegenerate quadratic tangency for $\mu=0$. Since the trivial solution $U=0$ persists for all $\mu$, we find that the two nonlinear manifolds acquire a second nontrivial intersection for $\mu>0$ which corresponds to the desired localized nontrivial radial spot, see Figure 2(a).

The outline of the rest of this paper is as follows. In $\S 2$ and $\S 3$, we discuss the equations near the core and in the far field, respectively. We then prove Theorems 1-3 in $\S 4-6$, respectively. In $\S 7$ and 8 , we present numerical computations of rings and spots for not necessarily small values of $\mu$. Conclusions and open problems are stated in $\S 9$. 


\section{The equation near the core}

Stationary radial solutions of the Swift-Hohenberg equation satisfy (1.2)

$$
\left(\partial_{r}^{2}+\frac{1}{r} \partial_{r}+1\right)^{2} u_{1}=-\mu u_{1}+\nu u_{1}^{2}-\kappa u_{1}^{3}
$$

which we rewrite as

$$
\begin{aligned}
\left(\partial_{r}^{2}+\frac{1}{r} \partial_{r}+1\right) u_{1} & =u_{2} \\
\left(\partial_{r}^{2}+\frac{1}{r} \partial_{r}+1\right) u_{2} & =-\mu u_{1}+\nu u_{1}^{2}-\kappa u_{1}^{3} .
\end{aligned}
$$

For the sake of simplicity, we shall omit in the rest of this paper the $\mathrm{O}\left(u^{4}\right)$-terms that may be present in the nonlinearity $f(u)$; their presence would not change any of the subsequent arguments.

Our approach follows [36]. We begin our analysis by characterizing all small radial solutions of (2.2) that are bounded and smooth in the interval $\left[0, r_{0}\right]$ for any fixed finite $r_{0}>0$. In particular, we are interested in solutions that are smooth in the limit $r \rightarrow 0$. Rewriting (2.2) as a first-order system, it suffices to find all solutions of

$$
U_{r}=\mathcal{A} U+\mathcal{F}(U, \mu), \quad \mathcal{A}=\left(\begin{array}{rrrr}
0 & 0 & 1 & 0 \\
0 & 0 & 0 & 1 \\
-1 & 1 & -\frac{1}{r} & 0 \\
0 & -1 & 0 & -\frac{1}{r}
\end{array}\right), \quad \mathcal{F}(U, \mu)=\left(\begin{array}{c}
0 \\
0 \\
0 \\
-\mu u_{1}+\nu u_{1}^{2}-\kappa u_{1}^{3}
\end{array}\right)
$$

that are bounded in the interval $\left[0, r_{0}\right]$ for any fixed finite $r_{0}>0$.

First, we set $\mu=0$ and linearize (2.3) about $U=0$ to get the linear system $U_{r}=\mathcal{A} U$ which has the four linearly independent solutions

$$
\begin{aligned}
& V_{1}(r)=\sqrt{2 \pi}\left(J_{0}(r), 0,-J_{1}(r), 0\right)^{T}, \quad V_{2}(r)=\sqrt{2 \pi}\left(r J_{1}(r), 2 J_{0}(r), r J_{0}(r),-2 J_{1}(r)\right)^{T} \\
& V_{3}(r)=\sqrt{2 \pi}\left(Y_{0}(r), 0,-Y_{1}(r), 0\right)^{T}, \quad V_{4}(r)=\sqrt{2 \pi}\left(r Y_{1}(r), 2 Y_{0}(r), r Y_{0}(r),-2 Y_{1}(r)\right)^{T},
\end{aligned}
$$

where $J_{k}$ and $Y_{k}$ denote the $k$ th Bessel functions of the first and second kind, respectively. These solutions can be found by inspecting the equivalent system

$$
\left(\partial_{r}^{2}+\frac{1}{r} \partial_{r}+1\right) u_{1}=u_{2}, \quad\left(\partial_{r}^{2}+\frac{1}{r} \partial_{r}+1\right) u_{2}=0 .
$$

Table 1 summarizes known expansions of the Bessel functions in the limits $r \rightarrow 0$ and $r \rightarrow \infty$. Thus, $V_{1}(r)$ and $V_{2}(r)$ stay bounded as $r \rightarrow 0$, while the norms of $V_{3}(r)$ and $V_{4}(r)$ behave like $\log r$ as $r \rightarrow 0$. We therefore expect that the set of solutions of (2.3) that are bounded as $r \rightarrow 0$ forms a two-dimensional manifold in $\mathbb{R}^{4}$ for each fixed $r>0$. We denote by $P_{-}^{c u}\left(r_{0}\right)$ the projection onto the space spanned by $V_{1,2}\left(r_{0}\right)$ with null space given by the span of $V_{3,4}\left(r_{0}\right)$.

\begin{tabular}{|c|c|c|}
\hline & $r \rightarrow 0$ & $r \rightarrow \infty$ \\
\hline$J_{0}$ & $1+\mathrm{O}\left(r^{2}\right)$ & $\sqrt{\frac{2}{\pi r}} \cos \left(r-\frac{\pi}{4}\right)+\mathrm{O}\left(r^{-\frac{3}{2}}\right)$ \\
$J_{1}$ & $r\left(\frac{1}{4}+\mathrm{O}\left(r^{2}\right)\right)$ & $\sqrt{\frac{2}{\pi r}} \sin \left(r-\frac{\pi}{4}\right)+\mathrm{O}\left(r^{-\frac{3}{2}}\right)$ \\
$Y_{0}$ & $\frac{2}{\pi}\left(1+\mathrm{O}\left(r^{2}\right)\right) \log r+\mathrm{O}(1)$ & $\sqrt{\frac{2}{\pi r}} \sin \left(r-\frac{\pi}{4}\right)+\mathrm{O}\left(r^{-\frac{3}{2}}\right)$ \\
$Y_{1}$ & $\frac{2}{\pi}\left(1+\mathrm{O}\left(r^{2}\right)\right) r \log r-\frac{2}{\pi r}+\mathrm{O}(1)$ & $-\sqrt{\frac{2}{\pi r}} \cos \left(r-\frac{\pi}{4}\right)+\mathrm{O}\left(r^{-\frac{3}{2}}\right)$ \\
\hline
\end{tabular}

Table 1: Expansions of the Bessel functions $J_{k}$ and $Y_{k}$ for $r \rightarrow 0$ and $r \rightarrow \infty$; see [1, (9.1.10), (9.1.11) and §9.2]. The remainder terms $\mathrm{O}(1)$ and $\mathrm{O}\left(r^{2}\right)$ in the left column have well-defined Taylor series in $r^{2}$, which can be differentiated term-by-term. 
Throughout this paper, we shall use the Landau symbols $\mathrm{O}(\ldots)$ and $\mathrm{O}_{r_{0}}(\ldots)$ with their usual meaning: the difference between the two symbols is that the constants that bound $\mathrm{O}(\ldots)$ can be chosen independently of $r_{0}$, while the constants that bound $\mathrm{O}_{r_{0}}(\ldots)$ may depend on $r_{0}$.

Lemma 1 Fix $r_{0}>0$, then there are constants $\delta_{0}, \delta_{1}>0$ so that the set $W_{-}^{\mathrm{cu}}(\mu)$ of solutions $U(r)$ of (2.3) for which $\sup _{0 \leq r \leq r_{0}}|U(r)|<\delta_{0}$ is, for $|\mu|<\delta_{0}$, a smooth two-dimensional manifold. Furthermore, $U \in W_{-}^{\text {cu }}(\mu)$ with $\left|P_{-}^{\mathrm{cu}}\left(r_{0}\right) U\left(r_{0}\right)\right|<\delta_{1}$ if and only if

$$
\begin{aligned}
U\left(r_{0}\right)= & \tilde{d}_{1} V_{1}\left(r_{0}\right)+\tilde{d}_{2} V_{2}\left(r_{0}\right)+V_{3}\left(r_{0}\right) \mathrm{O}_{r_{0}}\left(|\mu||\tilde{d}|+|\tilde{d}|^{2}\right) \\
& +V_{4}\left(r_{0}\right)\left(\left[\frac{1}{\sqrt{3}}+\mathrm{o}(1)\right] \nu \tilde{d}_{1}^{2}+\mathrm{O}_{r_{0}}\left(|\mu||\tilde{d}|+\left|\tilde{d}_{1}\right|^{3}+\left|\tilde{d}_{2}\right|^{2}\right)\right)
\end{aligned}
$$

for some $\tilde{d}=\left(\tilde{d}_{1}, \tilde{d}_{2}\right) \in \mathbb{R}^{2}$ with $|\tilde{d}|<\delta_{1}$, where the right-hand side in (2.5) depends smoothly on $(\tilde{d}, \mu)$, and o(1) is the Landau symbol in $r_{0}$ as $r_{0} \rightarrow \infty$.

Proof. We observe that four independent solutions to the adjoint system $U_{r}=-\mathcal{A}^{T} U$ are given by

$$
\begin{array}{cc}
W_{1}(r)=\frac{\sqrt{2 \pi}}{8}\left(-2 r Y_{1}(r), r^{2} Y_{0}(r),-2 r Y_{0}(r),-r^{2} Y_{1}(r)\right)^{T}, \quad W_{2}(r)=\frac{\sqrt{2 \pi}}{8}\left(0,-r Y_{1}(r), 0,-r Y_{0}(r)\right)^{T} \\
W_{3}(r)=\frac{\sqrt{2 \pi}}{8}\left(2 r J_{1}(r),-r^{2} J_{0}(r), 2 r J_{0}(r), r^{2} J_{1}(r)\right)^{T}, \quad W_{4}(r)=\frac{\sqrt{2 \pi}}{8}\left(0, r J_{1}(r), 0, r J_{0}(r)\right)^{T} .
\end{array}
$$

It follows from $[1,(9.1 .16)]$ that

$$
\left\langle V_{i}(r), W_{j}(r)\right\rangle_{\mathbb{R}^{4}}=\delta_{i j}, \quad i, j=1, \ldots, 4
$$

is independent of $r$. For given $\tilde{d}=\left(\tilde{d}_{1}, \tilde{d}_{2}\right) \in \mathbb{R}^{2}$, we consider the fixed-point equation

$$
\begin{aligned}
U(r) & =\sum_{j=1}^{2} \tilde{d}_{j} V_{j}(r)+\sum_{j=1}^{2} V_{j}(r) \int_{r_{0}}^{r}\left\langle W_{j}(s), \mathcal{F}(U(s), \mu)\right\rangle \mathrm{d} s+\sum_{j=3}^{4} V_{j}(r) \int_{0}^{r}\left\langle W_{j}(s), \mathcal{F}(U(s), \mu)\right\rangle \mathrm{d} s \\
& =\sum_{j=1}^{2} \tilde{d}_{j} V_{j}(r)+\sum_{j=1}^{2} V_{j}(r) \int_{r_{0}}^{r} W_{j, 4}(s) \mathcal{F}_{4}(U(s), \mu) \mathrm{d} s+\sum_{j=3}^{4} V_{j}(r) \int_{0}^{r} W_{j, 4}(s) \mathcal{F}_{4}(U(s), \mu) \mathrm{d} s
\end{aligned}
$$

on $C^{0}\left(\left[0, r_{0}\right], \mathbb{R}^{4}\right)$, where $W_{j, 4}(r)$ denotes the fourth component of $W_{j}(r)$.

First, each solution $U \in C^{0}\left(\left[0, r_{0}\right], \mathbb{R}^{4}\right)$ of $(2.7)$ gives a solution of $(2.3)$ that is bounded on $\left[0, r_{0}\right]$ : indeed, in the limit $r \rightarrow 0$, the terms $W_{j, 4}(r)$ are bounded by $r \log r$ and the integrals multiplying the unbounded solutions $V_{3,4}(r)$ are therefore bounded by $r^{2} \log r$ as $r \rightarrow 0$. Since $\left|V_{3,4}(r)\right|=\mathrm{O}(1 / r)$ as $r \rightarrow 0$, we see that the right-hand side of (2.7) lies in $C^{1}\left(\left[0, r_{0}\right], \mathbb{R}^{4}\right)$, whenever $U \in C^{0}\left(\left[0, r_{0}\right], \mathbb{R}^{4}\right)$, and it is easy to see that $U(r)$ satisfies $(2.3)$ on $\left[0, r_{0}\right]$.

Conversely, every bounded solution $U \in C^{0}\left(\left[0, r_{0}\right], \mathbb{R}^{4}\right)$ of $(2.3)$ satisfies $(2.7)$ provided we add $\tilde{d}_{3} V_{3}(r)+\tilde{d}_{4} V_{4}(r)$ to the right-hand side for an appropriate $\tilde{d} \in \mathbb{R}^{4}$. Recall that the integral terms on the right-hand side are bounded as $r \rightarrow 0$ whenever $U \in C^{0}\left(\left[0, r_{0}\right], \mathbb{R}^{4}\right)$. It is straightforward to check though that the term $\tilde{d}_{3} V_{3}(r)+\tilde{d}_{4} V_{4}(r)$ is unbounded as $r \rightarrow 0$ unless $\tilde{d}_{3}=\tilde{d}_{4}=0$, which proves the assertion.

It remains to solve (2.7), which can be done by applying the uniform contraction mapping principle for sufficiently small $\left(\tilde{d}_{1}, \tilde{d}_{2}\right)$ and $\mu$. The resulting solution $U$ satisfies

$$
U(r)=\sum_{j=1}^{2} \tilde{d}_{j} V_{j}(r)+\mathrm{O}_{r_{0}}\left(|\mu||\tilde{d}|+|\tilde{d}|^{2}\right)
$$

on $\left[0, r_{0}\right]$. If we evaluate $(2.7)$ at $r=r_{0}$, we arrive at $(2.5)$ except that we need to calculate the quadratic coefficient in $\tilde{d}_{1}$ in front of $V_{4}\left(r_{0}\right)$ : using a Taylor expansion, we find that this coefficient is given by

$$
\int_{0}^{r_{0}} W_{4,4}(s) \nu V_{1,1}(s)^{2} \mathrm{~d} s=\frac{\pi \nu}{4} \int_{0}^{r_{0}} s J_{0}(s)^{3} \mathrm{~d} s=\frac{\pi \nu}{4}\left[\int_{0}^{\infty} s J_{0}(s)^{3} \mathrm{~d} s+\mathrm{o}(1)\right]=\nu\left[\frac{1}{\sqrt{3}}+\mathrm{o}(1)\right],
$$

where we used $[43,(3)$ on p 411] to evaluate the last integral. This completes the proof of the lemma. 


\section{The far-field equations}

In this section, we look into the far-field regime where the radial variable $r$ is large. Recall from $\S 2$ that stationary radial solutions of the Swift-Hohenberg equation satisfy the first-order system (2.3) given by

$$
U_{r}=\mathcal{A} U+\mathcal{F}(U, \mu), \quad \mathcal{A}=\left(\begin{array}{rrrr}
0 & 0 & 1 & 0 \\
0 & 0 & 0 & 1 \\
-1 & 1 & -\frac{1}{r} & 0 \\
0 & -1 & 0 & -\frac{1}{r}
\end{array}\right), \quad \mathcal{F}(U, \mu)=\left(\begin{array}{c}
0 \\
0 \\
0 \\
-\mu u_{1}+\nu u_{1}^{2}-\kappa u_{1}^{3}
\end{array}\right)
$$

with $U=\left(u_{1}, u_{2}, u_{3}, u_{4}\right)^{T}$. It is convenient to make this equation autonomous by adding the variable $\alpha=1 / r$ which satisfies the equation $\alpha_{r}=-\alpha^{2}$. Equation (3.1) then becomes

$$
\frac{\mathrm{d}}{\mathrm{d} r}\left(\begin{array}{c}
u_{1} \\
u_{2} \\
u_{3} \\
u_{4} \\
\alpha
\end{array}\right)=\left(\begin{array}{c}
u_{3} \\
u_{4} \\
-u_{1}-\alpha u_{3}+u_{2} \\
-u_{2}-\alpha u_{4}-\mu u_{1}+\nu u_{1}^{2}-\kappa u_{1}^{3} \\
-\alpha^{2}
\end{array}\right) .
$$

In the remainder of this section, we focus on the regime $0<\alpha \ll 1$ which corresponds to the far field $r \gg 1$. As in $[13,36]$, we use the linear normal-form coordinates

$$
U=\tilde{A}\left(\begin{array}{c}
1 \\
0 \\
\mathrm{i} \\
0
\end{array}\right)+\tilde{B}\left(\begin{array}{r}
0 \\
2 \mathrm{i} \\
1 \\
-2
\end{array}\right)+\text { c.c. }
$$

or, equivalently,

$$
\left(\begin{array}{c}
\tilde{A} \\
\tilde{B}
\end{array}\right)=\frac{1}{4}\left(\begin{array}{c}
2 u_{1}-\mathrm{i}\left(2 u_{3}+u_{4}\right) \\
-u_{4}-\mathrm{i} u_{2}
\end{array}\right), \quad U=\left(u_{1}, u_{2}, u_{3}, u_{4}\right)^{T} .
$$

In these coordinates, (3.2) becomes

$$
\begin{aligned}
\tilde{A}_{r} & =\left(\mathrm{i}-\frac{\alpha}{2}\right) \tilde{A}+\tilde{B}+\frac{\alpha}{2} \overline{\tilde{A}}+\mathrm{O}((|\mu|+|\tilde{A}|+|\tilde{B}|)(|\tilde{A}|+|\tilde{B}|)) \\
\tilde{B}_{r} & =\left(\mathrm{i}-\frac{\alpha}{2}\right) \tilde{B}-\frac{\alpha}{2} \tilde{B}+\mathrm{O}((|\mu|+|\tilde{A}|+|\tilde{B}|)(|\tilde{A}|+|\tilde{B}|)) \\
\alpha_{r} & =-\alpha^{2} .
\end{aligned}
$$

Nonlinear normal-form transformations can now be used to remove non-resonant terms to any given finite order from the right-hand side.

Lemma 2 Fix $0<m<\infty$, then there exists a change of coordinates

$$
\left(\begin{array}{c}
A \\
B
\end{array}\right)=\mathrm{e}^{-\mathrm{i} \phi(r)}[1+\mathcal{T}(\alpha)]\left(\begin{array}{c}
\tilde{A} \\
\tilde{B}
\end{array}\right)+\mathrm{O}((|\mu|+|\tilde{A}|+|\tilde{B}|)(|\tilde{A}|+|\tilde{B}|))
$$

so that (3.5) becomes

$$
\begin{aligned}
A_{r} & =-\frac{\alpha}{2} A+B+\mathcal{R}_{A}(A, B, \alpha, \mu) \\
B_{r} & =-\frac{\alpha}{2} B+\frac{1}{4} \mu A+c_{3}^{0}|A|^{2} A+\mathcal{R}_{B}(A, B, \alpha, \mu) \\
\alpha_{r} & =-\alpha^{2} .
\end{aligned}
$$


The coordinate change (3.6) is polynomial in $(A, B, \alpha)$ and smooth in $\mu$, and $\mathcal{T}(\alpha)=\mathrm{O}(\alpha)$ is linear and upper triangular for each $\alpha$, while $\phi(r)$ satisfies

$$
\phi_{r}=1+\mathrm{O}\left(\mu+|\alpha|^{3}+|A|^{2}\right), \quad \phi(0)=0 .
$$

The constant $c_{3}^{0}$ is given by $c_{3}^{0}=3 \kappa / 4-19 \nu^{2} / 18$, and the remainder terms satisfy

$$
\begin{aligned}
& \mathcal{R}_{A}(A, B, \alpha, \mu)=\mathrm{O}\left(\sum_{j=0}^{2}\left|A^{j} B^{3-j}\right|+|\alpha|^{3}|A|+|\alpha|^{2}|B|+(|A|+|B|)^{5}+|\mu||\alpha|^{m}(|A|+|B|)\right) \\
& \mathcal{R}_{B}(A, B, \alpha, \mu)=\mathrm{O}\left(\sum_{j=0}^{1}\left|A^{j} B^{3-j}\right|+|\alpha|^{3}|B|+|\mu|\left(|\mu|+|\alpha|^{3}+|A|^{2}\right)|A|+(|A|+|B|)^{5}+|\mu||\alpha|^{m}|B|\right) .
\end{aligned}
$$

Proof. It follows from [36, Lemma 3.10 and Corollary 3.14] that we can bring (3.5) into the normal form

$$
\begin{aligned}
A_{r}= & \left(\mathrm{i} k_{1}(\alpha, \mu)+k_{2}(\alpha, \mu)\right) A+B+\mathrm{i} c_{2}(\alpha, \mu)|A|^{2} A \\
& +\mathrm{O}\left(\sum_{j=0}^{2}\left|A^{j} B^{3-j}\right|+(|A|+|B|)^{5}+|\alpha|^{2}|B|+|\mu||\alpha|^{m}(|A|+|B|)\right) \\
B_{r}= & \left(\mathrm{i} k_{1}(\alpha, \mu)+k_{2}(\alpha, \mu)\right) B+c_{1}(\alpha, \mu) A+c_{3}(\alpha, \mu)|A|^{2} A+\mathrm{i} c_{2}(\alpha, \mu)|A|^{2} B \\
& +\mathrm{O}\left(\sum_{j=0}^{1}\left|A^{j} B^{3-j}\right|+(|A|+|B|)^{5}+|\mu||\alpha|^{m}(|A|+|B|)\right) \\
\alpha_{r}= & -\alpha^{2}
\end{aligned}
$$

by a transformation

$$
\left(\begin{array}{c}
A \\
B
\end{array}\right)=[1+\mathcal{T}(\alpha)]\left(\begin{array}{c}
\tilde{A} \\
\tilde{B}
\end{array}\right)+\mathrm{O}((|\mu|+|\tilde{A}|+|\tilde{B}|)(|\tilde{A}|+|\tilde{B}|))
$$

which is polynomial in $(\tilde{A}, \tilde{B}, \alpha)$, smooth in $\mu$, and $\mathrm{O}(|\alpha|+|\mu|+|\tilde{A}|+|\tilde{B}|)$-close to the identity. We conclude from [36, Proof of Lemma 3.10 on p 44] that $\mathcal{T}(\alpha)$ is upper triangular, and we now briefly outline the reasoning. The transformation $\mathcal{T}(\alpha)$ puts the linear part of (3.5) for $\mu=0$ into normal form. Upon setting $\mu=0$ and ignoring the nonlinear terms in (3.5), it was shown in [36] that the $\bar{B}$-dependence in the equation (3.5) for $\tilde{B}$ can be removed by the transformation $B=\tilde{B}+g_{1}(r) \overline{\tilde{B}}$ for an appropriate function $g_{1}$; similarly, the subsequent transformation $A=\tilde{A}-g_{1}(r) \overline{\tilde{A}}+g_{2}(r) \overline{\tilde{B}}$ for an appropriate function $g_{2}$ puts the first equation into the desired form $A_{r}=[\ldots] A+\left[1+\mathrm{O}\left(\alpha^{2}\right)\right] B$. We remark that $\mathcal{T}(\alpha)$ is, to leading order, given by

$$
\left(\begin{array}{c}
A \\
B
\end{array}\right)=\left(\begin{array}{c}
\tilde{A}-\frac{\mathrm{i} \alpha}{4} \overline{\tilde{A}}-\frac{1}{4 r} \overline{\tilde{B}}+\mathrm{O}\left(\alpha^{2}\right)(\overline{\tilde{A}}, \overline{\tilde{B}}) \\
\tilde{B}+\frac{\mathrm{i} \alpha}{4} \tilde{B}+\mathrm{O}\left(\alpha^{2}\right) \tilde{\tilde{B}}
\end{array}\right) .
$$

Next, we claim that the coefficients $c_{1}, c_{3}, k_{1}$ and $k_{2}$ appearing in (3.10) are given by

$$
\begin{array}{ll}
c_{1}(\alpha, \mu)=\mu\left(\frac{1}{4}+\mathrm{O}\left(\mu+|\alpha|^{4}\right)\right), & c_{3}(\alpha, \mu)=\frac{3 \kappa}{4}-\frac{19 \nu^{2}}{18}+\mathrm{O}\left(|\mu|+|\alpha|^{4}\right) \\
k_{1}(\alpha, \mu)=1+\mathrm{O}\left(\mu+|\alpha|^{3}\right), & k_{2}(\alpha, \mu)=-\frac{\alpha}{2}+\mathrm{O}\left(|\alpha|^{3}\right) .
\end{array}
$$

Indeed, the leading-order terms for $\alpha=0$ were computed in [8], and the estimates for the remainder terms $c_{3}$, $k_{1}$ and $k_{2}$ follow again from [36, Lemma 3.10] and from the reversibility of (3.10) with respect to the reverser $(A, B, \alpha) \mapsto(\bar{A},-\bar{B},-\alpha))$. The linearization of $(3.5)$ about $(\tilde{A}, \tilde{B})=0$ leaves the subspace $\tilde{B}=0$ invariant when $\mu=0$. Since $\mathcal{T}$ is upper triangular, the same is true for the linearization of (3.10) at $\mu=0$, which implies that $c_{1}(\alpha, 0)=0$. 
In the next step, we remove the terms with purely imaginary factors from the right-hand side of (3.10). We define $\phi(r)$ to be the solution of

$$
\phi_{r}=k_{1}(\alpha, \mu)+c_{2}(\alpha, \mu)|A|^{2}=1+\mathrm{O}\left(\mu+|\alpha|^{3}+|A|^{2}\right), \quad \phi(0)=0
$$

and employ the transformation

$$
(A, B) \longmapsto \mathrm{e}^{\mathrm{i} \phi(r)}(A, B)
$$

which turns (3.10) into the desired form (3.7).

As we shall be interested in small solutions, we rescale $(A, B, \alpha)$ and the independent variable $r$ with the anticipated amplitude which is of the order $\sqrt{|\mu|}$. Throughout this paper, we consider exclusively the case $\mu \geq 0$ and define

$$
A=\sqrt{\mu} a, \quad B=\mu b, \quad r=\frac{s}{\sqrt{\mu}}
$$

for which (3.7) becomes

$$
\begin{aligned}
& a_{s}=b-\frac{a}{2 s}+\mathcal{R}_{1}(a, b, s, \mu) \\
& b_{s}=-\frac{b}{2 s}+\frac{a}{4}+c_{3}^{0}|a|^{2} a+\mathcal{R}_{2}(a, b, s, \mu)
\end{aligned}
$$

where

$$
\begin{aligned}
& \mathcal{R}_{1}(a, b, s, \mu)=\mu^{-1} \mathcal{R}_{A}(\sqrt{\mu} a, \mu b, \sqrt{\mu} / s, \mu)=\mathrm{O}(\mu(|a|+|b|)) \\
& \mathcal{R}_{2}(a, b, s, \mu)=\mu^{-\frac{3}{2}} \mathcal{R}_{B}(\sqrt{\mu} a, \mu b, \sqrt{\mu} / s, \mu)=\mathrm{O}(\mu(|a|+|b|))
\end{aligned}
$$

uniformly in $s \geq s_{1}$ for each fixed $s_{1}>0$. We shall also use the variables

$$
\left(\begin{array}{l}
a \\
b
\end{array}\right)=\frac{1}{\sqrt{s}}\left(\begin{array}{l}
\hat{a} \\
\hat{b}
\end{array}\right)
$$

in which (3.13) becomes

$$
\begin{aligned}
& \hat{a}_{s}=\hat{b}+\mathrm{O}(\mu(|\hat{a}|+|\hat{b}|)) \\
& \hat{b}_{s}=\frac{\hat{a}}{4}+c_{3}^{0}|\hat{a}|^{2} \hat{a}+\mathrm{O}(\mu(|\hat{a}|+|\hat{b}|)) .
\end{aligned}
$$

The estimates for the remainder terms given above are valid for $s \geq s_{1}$ for each fixed $s_{1}>0$. Note, however, that $r=r_{0}$ corresponds to $s=r_{0} \sqrt{\mu}$. To capture the region $r_{0} \sqrt{\mu} \leq s \leq s_{1}$, we use the variables

$$
\left(\begin{array}{c}
\tilde{a} \\
\tilde{b}
\end{array}\right)=\left(\begin{array}{c}
a \\
s\left(b-\frac{a}{2 s}\right)
\end{array}\right), \quad \tau=\log s
$$

from [36] so that $s=\mathrm{e}^{\tau}$, and $s \rightarrow 0$ corresponds to $\tau \rightarrow-\infty$. In these variables, (3.16) becomes

$$
\begin{aligned}
\tilde{a}_{\tau} & =\tilde{b}+\tilde{\mathcal{R}}_{1}(\tilde{a}, \tilde{b}, s, \mu) \\
\tilde{b}_{\tau} & =\frac{\tilde{a}}{4}+s^{2}\left(\frac{\tilde{a}}{4}+c_{3}^{0}|\tilde{a}|^{2} \tilde{a}\right)+\tilde{\mathcal{R}}_{2}(\tilde{a}, \tilde{b}, s, \mu) \\
s_{\tau} & =s
\end{aligned}
$$

with $(\tilde{a}, \tilde{b}, s) \in \mathbb{C}^{2} \times \mathbb{R}^{+}$.

Lemma 3 The remainder terms $\tilde{\mathcal{R}}_{j}(\tilde{a}, \tilde{b}, s, \mu)$ with $j=1,2$ are smooth in $(\tilde{a}, \tilde{b})$, continuous in $(s, \mu)$ for $s>0$, and have the expansion

$$
\tilde{\mathcal{R}}_{j}(\tilde{a}, \tilde{b}, s, \mu)=\mu \mathrm{O}\left(s^{-2}\right)(\tilde{a}, \tilde{b})+s \sqrt{\mu} \mathrm{O}(|\tilde{a}|+|\tilde{b}|), \quad s \geq \sqrt{\mu} r_{0}
$$

uniformly in $r_{0} \geq 1$ and $\mu \geq 0$. This estimate is also valid for the derivatives of $\tilde{\mathcal{R}}_{j}$ with respect to $(\tilde{a}, \tilde{b})$. 
We remark that the $\mu \mathrm{O}\left(s^{-2}\right)(\tilde{a}, \tilde{b})$ terms come from the terms $\mathrm{O}\left(\alpha^{3} A+\alpha^{2} B\right)$ and $\mathrm{O}\left(\alpha^{3} B\right)$ in (3.9), which are linear in $(A, B)$ and reflect the dependence on the radial variable $r$. These terms were neglected in [36, Eqn (73)] and the subsequent analysis.

Proof. The remainder terms $\tilde{\mathcal{R}}_{j}(\tilde{a}, \tilde{b}, s, \mu)$ are given by

$$
\tilde{\mathcal{R}}_{1}(\tilde{a}, \tilde{b}, s, \mu)=s \mathcal{R}_{1}(\tilde{a},(\tilde{b}+\tilde{a} / 2) / s, s, \mu), \quad \tilde{\mathcal{R}}_{2}(\tilde{a}, \tilde{b}, s, \mu)=s^{2} \mathcal{R}_{2}(\tilde{a},(\tilde{b}+\tilde{a} / 2) / s, s, \mu) .
$$

The claimed estimate can now be verified by inspecting the estimates (3.9) and (3.14). We omit the tedious but straightforward details.

Our goal will be to find nontrivial intersections of the stable manifold $W_{+}^{\mathrm{s}}(\mu)$, which consists of all solutions that decay as $r \rightarrow \infty$, with the center-unstable manifold $W_{-}^{\mathrm{cu}}(\mu)$, which consists of solutions that stay smooth at the core $r=0$.

It is convenient to use the $(A, B)$-variables to find such intersections, and we therefore transform the expression for the center-unstable manifold $W_{-}^{\mathrm{cu}}(\mu)$ into the $(A, B)$-coordinates. To this end, we write the expansion (2.5) for each fixed $r_{0} \gg 1$ in the $(\tilde{A}, \tilde{B})$-coordinates (3.4) and afterwards in the coordinates $(A, B)$ from (3.6). Substituting (2.4) evaluated at $r=r_{0}$ into (3.4) and using Table 1, we find that

$$
\begin{array}{cl}
\left(\begin{array}{c}
\tilde{A}_{1} \\
\tilde{B}_{1}
\end{array}\right)=\frac{1}{\sqrt{r_{0}}} \mathrm{e}^{\mathrm{i}\left(r_{0}-\pi / 4\right)}\left(\begin{array}{c}
1+\mathrm{O}\left(r_{0}^{-1}\right) \\
0
\end{array}\right), & \left(\begin{array}{c}
\tilde{A}_{2} \\
\tilde{B}_{2}
\end{array}\right)=\sqrt{r_{0}} \mathrm{e}^{\mathrm{i}\left(r_{0}-\pi / 4\right)}\left(\begin{array}{c}
-\mathrm{i}+\mathrm{O}\left(r_{0}^{-1}\right) \\
-\frac{\mathrm{i}}{r_{0}}+\mathrm{O}\left(r_{0}^{-2}\right)
\end{array}\right) \\
\left(\begin{array}{c}
\tilde{A}_{3} \\
\tilde{B}_{3}
\end{array}\right)=\frac{1}{\sqrt{r_{0}}} \mathrm{e}^{\mathrm{i}\left(r_{0}-\pi / 4\right)}\left(\begin{array}{c}
-\mathrm{i}+\mathrm{O}\left(r_{0}^{-1}\right) \\
0
\end{array}\right), & \left(\begin{array}{c}
\tilde{A}_{4} \\
\tilde{B}_{4}
\end{array}\right)=\sqrt{r_{0}} \mathrm{e}^{\mathrm{i}\left(r_{0}-\pi / 4\right)}\left(\begin{array}{c}
-1+\mathrm{O}\left(r_{0}^{-1}\right) \\
-\frac{1}{r_{0}}+\mathrm{O}\left(r_{0}^{-2}\right)
\end{array}\right),
\end{array}
$$

where $\left(\tilde{A}_{j}, \tilde{B}_{j}\right)$ corresponds to $V_{j}\left(r_{0}\right)$ for $j=1, \ldots, 4$. Using the variables

$$
\left(d_{1}, d_{2}\right)=\left(\tilde{d}_{1} / \sqrt{r_{0}}, \sqrt{r_{0}} \tilde{d}_{2}\right),
$$

we arrive at the expression

$$
\left(\begin{array}{c}
\tilde{A} \\
\tilde{B}
\end{array}\right)=\mathrm{e}^{\mathrm{i}\left(r_{0}-\pi / 4\right)}\left(\begin{array}{c}
d_{1}\left[1+\mathrm{O}\left(r_{0}^{-1}\right)\right]-d_{2}\left[\mathrm{i}+\mathrm{O}\left(r_{0}^{-1}\right)\right]+\mathrm{O}_{r_{0}}\left(|\mu||d|+|d|^{2}\right) \\
-d_{2} r_{0}^{-1}\left[\mathrm{i}+\mathrm{O}\left(r_{0}^{-1}\right)\right]-[1 / \sqrt{3}+\mathrm{o}(1)] \sqrt{r_{0}} \nu d_{1}^{2}+\mathrm{O}_{r_{0}}\left(|\mu||d|+\left|d_{2}\right|^{2}+\left|d_{1}\right|^{3}\right)
\end{array}\right)
$$

for $U\left(r_{0}\right)$ from (2.5). We are now ready to express the $r=r_{0}$ slice of $W_{-}^{\text {cu }}(\mu)$ in the coordinates $(A, B)$ given in (3.6). From (3.8), we find

$$
\phi\left(r_{0}\right)=r_{0}+\mathrm{O}\left(1 / r_{0}^{2}\right)+\mathrm{O}_{r_{0}}\left(|\mu|+|d|^{2}\right) .
$$

Applying the transformation (3.6), we then obtain the expression

$$
\begin{aligned}
\left.W_{-}^{\mathrm{cu}}(\mu)\right|_{r=r_{0}}:\left(\begin{array}{c}
A \\
B
\end{array}\right)= & \mathrm{e}^{\mathrm{i}\left[-\pi / 4+\mathrm{O}\left(1 / r_{0}^{2}\right)+\mathrm{O}_{r_{0}}\left(|\mu|+|d|^{2}\right)\right]} \times \\
& \times\left(\begin{array}{c}
d_{1}\left[1+\mathrm{O}\left(r_{0}^{-1}\right)\right]-d_{2}\left[\mathrm{i}+\mathrm{O}\left(r_{0}^{-1}\right)\right]+\mathrm{O}_{r_{0}}\left(|\mu||d|+|d|^{2}\right) \\
-d_{2} r_{0}^{-1}\left[\mathrm{i}+\mathrm{O}\left(r_{0}^{-1}\right)\right]-[1 / \sqrt{3}+\mathrm{o}(1)] \sqrt{r_{0}} \nu d_{1}^{2}+\mathrm{O}_{r_{0}}\left(|\mu||d|+\left|d_{2}\right|^{2}+\left|d_{1}\right|^{3}\right)
\end{array}\right)
\end{aligned}
$$

for $U\left(r_{0}\right)$, where we exploited the facts that $\mathcal{T}(\alpha)$ is upper triangular and that the coefficient in front of $d_{1}^{2}$ in the $\tilde{B}$-component scales with $\sqrt{r_{0}}$ so that it is affected only at higher order by the quadratic terms in the transformation (3.6).

\section{Planar ring solutions}

In this section, we prove Theorem 1. We begin by considering the far-field equation (3.16)

$$
\begin{aligned}
& \hat{a}_{s}=\hat{b}+\mathrm{O}(\mu(|\hat{a}|+|\hat{b}|)) \\
& \hat{b}_{s}=\frac{\hat{a}}{4}+c_{3}^{0}|\hat{a}|^{2} \hat{a}+\mathrm{O}(\mu(|\hat{a}|+|\hat{b}|))
\end{aligned}
$$




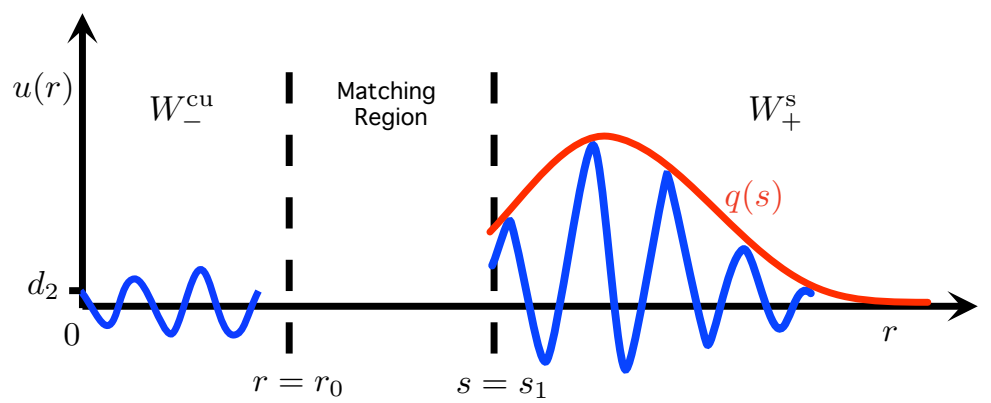

Figure 5: Schematic illustration of the matching regions for rings.

where $s \geq s_{1}$ for any fixed $s_{1}>0$ and $(\hat{a}, \hat{b}) \in \mathbb{C}^{2}$. The origin $(\hat{a}, \hat{b})=0$ is a hyperbolic equilibrium of the nonautonomous equation (4.1) for all $\mu \geq 0$. It therefore has a smooth two-dimensional ${ }^{1}$ stable manifold $W_{+}^{\mathrm{s}}(\mu)$ which consists of all solutions $(\hat{a}, \hat{b})$ of $(4.1)$ which converge to zero as $s \rightarrow \infty$. Such solutions necessarily decay exponentially in $s$, and $W_{+}^{\mathrm{s}}(\mu)$ is continuous in $\mu \geq 0$ and smooth in $\sqrt{\mu}$. In particular, we can expand $W_{+}^{\mathrm{s}}(\mu)$ at $s=s_{1}$ in terms of $(\hat{a}, \hat{b}, \sqrt{\mu})$.

Our goal is to find nontrivial intersections of $W_{+}^{\mathrm{s}}(\mu)$ and the core manifold $W_{-}^{\mathrm{cu}}(\mu)$ which give rise to localized radial solutions of the Swift-Hohenberg equation. We choose to seek such intersections at $r=r_{0}$ which corresponds to $s=\sqrt{\mu} r_{0}$. Thus, we need to control the stable manifold $W_{+}^{\mathrm{s}}(\mu)$ for $s \geq \sqrt{\mu} r_{0}$ : note that the estimates for the remainder terms in (4.1) are valid only for $s \geq s_{1}$ for each fixed $s_{1}>0$. To control $W_{+}^{\mathrm{s}}(\mu)$ for $\sqrt{\mu} r_{0} \leq s \leq s_{1}$, we use the variables (3.17) for which (4.1) becomes

$$
\begin{aligned}
& \tilde{a}_{\tau}=\tilde{b}+\tilde{\mathcal{R}}_{1}(\tilde{a}, \tilde{b}, s, \mu) \\
& \tilde{b}_{\tau}=\frac{\tilde{a}}{4}+s^{2}\left(\frac{\tilde{a}}{4}+c_{3}^{0}|\tilde{a}|^{2} \tilde{a}\right)+\tilde{\mathcal{R}}_{2}(\tilde{a}, \tilde{b}, s, \mu) \\
& s_{\tau}=s
\end{aligned}
$$

where

$$
\tilde{\mathcal{R}}_{j}(\tilde{a}, \tilde{b}, s, \mu)=\mu \mathrm{O}\left(s^{-2}\right)(\tilde{a}, \tilde{b})+s \sqrt{\mu} \mathrm{O}(|\tilde{a}|+|\tilde{b}|), \quad s \geq \sqrt{\mu} r_{0}
$$

uniformly in $r_{0} \geq 1$ and $\mu \geq 0$; see Lemma 3 .

We shall first ignore the remainder terms in (4.1) and consider the system

$$
\begin{aligned}
& \hat{a}_{s}=\hat{b} \\
& \hat{b}_{s}=\frac{\hat{a}}{4}+c_{3}^{0}|\hat{a}|^{2} \hat{a}
\end{aligned}
$$

for $s>0$. In the original $(a, b)$ variables, (4.4) corresponds to the nonautonomous second-order equation

$$
a_{s s}=-\frac{a_{s}}{s}+\frac{a}{4 s^{2}}+\frac{a}{4}+c_{3}^{0}|a|^{2} a .
$$

Lemma 4 Assume that $c_{3}^{0}<0$, then, for each integer $n \geq 0$, equation (4.5) has a bounded nontrivial real solution $q_{n}(s)$ that has precisely $n$ simple zeros for $s \in(0, \infty)$ and satisfies $q_{n}(s)=\mathrm{O}(\sqrt{s})$ as $s \rightarrow 0$ and $\left(q_{n}, q_{n}^{\prime}\right)(s) \rightarrow 0$ exponentially as $s \rightarrow \infty$. Furthermore, the linearization of (4.5) about $q_{n}(s)$ does not have a nontrivial real-valued solution that is bounded uniformly on $\mathbb{R}^{+}$. If $c_{3}^{0}>0$, then the only bounded solution of (4.5) on $\mathbb{R}^{+}$is a $(s) \equiv 0$.

Proof. The assertion for $c_{3}^{0}<0$ was proved in [35, Propositions 1-3] for the equation $a_{s s}+a_{s} / s-a k^{2} / s^{2}=a-a|a|^{2}$ with $k \in \mathbb{Z}$, but the proof works also without any changes for the case $k=\frac{1}{2}$. For $c_{3}^{0}>0$, we multiply (4.5) by $s \bar{a}(s)$ and integrate over $s \in(0, \infty)$ to find that the only possible solution is $a \equiv 0$ : it is easy to see that the resulting integrals exist whenever $a(s)$ is a bounded solution of (4.5).

\footnotetext{
${ }^{1}$ We always count real dimensions and omit the independent variable $s$ from dimension counts
} 
The nonexistence of ring solutions for $c_{3}^{0}>0$ follows from Lemma 4 . Thus, from now on, we assume that the coefficient $c_{3}^{0}$ satisfies $c_{3}^{0}<0$.

The functions $\left(\hat{a}_{n}, \hat{b}_{n}\right)=\sqrt{s}\left(q_{n}(s), q_{n}^{\prime}(s)\right)$ associated with $q_{n}(s)$ satisfy (4.1) with $\mu=0$ for $s \geq s_{1}$ and therefore lie in $W_{+}^{\text {s }}(0)$. We now consider the implications for (4.2): if we omit the remainder terms in (4.2), we arrive at the equation

$$
\begin{aligned}
\tilde{a}_{\tau} & =\tilde{b} \\
\tilde{b}_{\tau} & =\frac{\tilde{a}}{4}+s^{2}\left(\frac{\tilde{a}}{4}-\left|c_{3}^{0}\right||\tilde{a}|^{2} \tilde{a}\right) \\
s_{\tau} & =s
\end{aligned}
$$

which has the hyperbolic equilibrium $(\tilde{a}, \tilde{b}, s)=0$ with linearization

$$
\left(\begin{array}{c}
\tilde{a}_{\tau} \\
\tilde{b}_{\tau} \\
s_{\tau}
\end{array}\right)=\left(\begin{array}{ccc}
0 & 1 & 0 \\
\frac{1}{4} & 0 & 0 \\
0 & 0 & 1
\end{array}\right)\left(\begin{array}{c}
\tilde{a} \\
\tilde{b} \\
s
\end{array}\right)
$$

and unstable manifold $W_{-}^{\mathrm{u}}(0)$. Using the transformation (3.17) with the remainder term omitted, we see that the functions $\left(\tilde{a}_{n}, \tilde{b}_{n}\right)=\left(q_{n}(s), s q_{n}^{\prime}(s)-q_{n}(s) / 2\right)$ satisfy (4.6) with $s=\mathrm{e}^{\tau}$. Since $q_{n}(s)=\mathrm{O}(\sqrt{s})$, the solutions $\left(\tilde{a}_{n}, \tilde{b}_{n}\right)(\tau)=\mathrm{O}\left(\mathrm{e}^{\tau / 2}\right)$ lie in the unstable manifold $W_{-}^{\mathrm{u}}(0)$ of the equilibrium $(\tilde{a}, \tilde{b}, s)=0$ of $(4.6)$. The same arguments taken together with Lemma 4 show that $W_{-}^{\mathrm{u}}(0)$ and $W_{+}^{\mathrm{s}}(0)$ intersect transversely along $\left(\tilde{a}_{n}, \tilde{b}_{n}\right)(\tau)$ in the invariant real subspace of $\mathbb{C}^{2} \times \mathbb{R}^{+}$. Since (4.6) is equivariant under phase rotations, there is a one-parameter family $\mathrm{e}^{\mathrm{i} \eta_{2}}\left(\tilde{a}_{n}, \tilde{b}_{n}\right)(\tau)$ of homoclinic orbits, where $\eta_{2} \in \mathbb{R}$ is arbitrary. We now use this information to study (4.2) with the remainder terms $\tilde{\mathcal{R}}_{j}$ being present.

Lemma 5 For each fixed $r_{0} \gg 1$ and $n \in \mathbb{N}$, there exist numbers $q_{n}^{0} \neq 0$ and $\delta>0$ such that the $s=\sqrt{\mu} r_{0}$ fiber of the stable manifold $W_{+}^{\mathrm{s}}(\mu)$ of (4.2) near $\left\{\mathrm{e}^{\mathrm{i} \eta_{2}}\left(\tilde{a}_{n}, \tilde{b}_{n}\right)\left(\sqrt{\mu} r_{0}\right)\right\}$ is given by

$$
\left(\begin{array}{c}
\tilde{a} \\
\tilde{b}
\end{array}\right)=\mathrm{e}^{\mathrm{i} \eta_{2}}\left[\left(\mu^{\frac{1}{4}} r_{0}^{\frac{1}{2}} q_{n}^{0}\left[1+\mathrm{O}\left(r_{0}^{-2}\right)\right]+\mathrm{O}\left(r_{0}^{-2} \eta_{1}\right)+\mathrm{O}_{r_{0}}\left(\mu^{\frac{1}{2}}\right)\right)\left(\begin{array}{c}
1 \\
\frac{1}{2}
\end{array}\right)+\left(\eta_{1}+\mathrm{O}_{r_{0}}(\mu)\right)\left(\begin{array}{c}
1 \\
-\frac{1}{2}
\end{array}\right)\right]
$$

where $\left|\eta_{1}\right|<\delta, \eta_{2} \in \mathbb{R}$, and $0 \leq \mu<\delta$.

Proof. Recall that our goal is to track the stable manifold of (4.2) from $s=s_{1}$ along the homoclinic orbit $\left(\tilde{a}_{n}, \tilde{b}_{n}\right)$ back to $s=\sqrt{\mu} r_{0}$. For $\mu>0$, we may already lose control of the homoclinic orbit, since the perturbation $\mathrm{O}\left(\mu / s^{2}\right)=\mathrm{O}\left(r_{0}^{-2}\right)$ present in (4.2)-(4.3) does not go to zero when $\mu$ tends to zero. Thus, we first construct a solution of (4.2) near the homoclinic orbit. Afterwards, we solve the equation near this newly constructed solution and use the resulting semiflow to track the stable manifold back to $s=\sqrt{\mu} r_{0}$.

Let $\tau_{0}=\log \left(\sqrt{\mu} r_{0}\right)$ and $\tau_{1}=\log s_{1}$, where $0<s_{1} \ll 1$ is small but fixed, and note that $\tau_{j}<0$. Throughout the proof, we consider (4.2),

$$
\left(\begin{array}{c}
\tilde{a}_{\tau} \\
\tilde{b}_{\tau}
\end{array}\right)=\left(\begin{array}{c}
\tilde{b} \\
\tilde{a}+s^{2}\left(\frac{\tilde{a}}{4}+c_{3}^{0}|\tilde{a}|^{2} \tilde{a}\right)
\end{array}\right)+\mu \mathrm{O}\left(s^{-2}\right)(\tilde{a}, \tilde{b})+s \sqrt{\mu} \mathrm{O}(|\tilde{a}|+|\tilde{b}|),
$$

with $s=\mathrm{e}^{\tau}$ for $\tau$ in $\left[\tau_{0}, \tau_{1}\right]$, which corresponds to $s \in\left[\sqrt{\mu} r_{0}, s_{1}\right]$. We introduce the coordinates

$$
u=\left(\begin{array}{l}
u_{1} \\
u_{2}
\end{array}\right)=\frac{1}{2}\left(\begin{array}{rr}
1 & 2 \\
1 & -2
\end{array}\right)\left(\begin{array}{l}
\tilde{a} \\
\tilde{b}
\end{array}\right), \quad\left(\begin{array}{l}
\tilde{a} \\
\tilde{b}
\end{array}\right)=\left(\begin{array}{rr}
1 & 1 \\
\frac{1}{2} & -\frac{1}{2}
\end{array}\right)\left(\begin{array}{l}
u_{1} \\
u_{2}
\end{array}\right)
$$

in which (4.8) becomes

$$
u_{\tau}=\left[D+\mathrm{O}\left(\mu \mathrm{e}^{-2 \tau}\right)\right] u+\mathrm{O}\left(\left(\sqrt{\mu}+\mathrm{e}^{\tau}\right) \mathrm{e}^{\tau}|u|\right), \quad D=\left(\begin{array}{rr}
\frac{1}{2} & 0 \\
0 & -\frac{1}{2}
\end{array}\right)
$$


We denote by $Q_{n}^{0}(\tau)$ the function corresponding to the homoclinic orbit $\left(\tilde{a}_{n}, \tilde{b}_{n}\right)(\tau)$. In particular, $Q_{n}^{0}(\tau)$ satisfies (4.10) for $\tau \in \mathbb{R}$ provided we omit all remainder terms; using an appropriate integral equation, it is not difficult to prove that

$$
Q_{n}^{0}(\tau)=\mathrm{e}^{\tau / 2}\left(\begin{array}{c}
q_{n}^{0} \\
0
\end{array}\right)+\mathrm{O}\left(\mathrm{e}^{2 \tau}\left|q_{n}^{0}\right|\right)
$$

for some $q_{n}^{0}>0$, uniformly in $\tau \leq \tau_{1}$. We first construct a solution $Q_{n}$ of the full equation (4.10) that is close to $Q_{n}^{0}$ for $s \geq \sqrt{\mu} r_{0}$ for all $\mu$ sufficiently close to zero. Thus, we set $u=Q_{n}^{0}+v$ and, using that $Q_{n}^{0}$ satisfies (4.10) without the remainder terms, arrive at the equation

$$
\begin{aligned}
v_{\tau} & =\left[D+\mathrm{O}\left(\mu \mathrm{e}^{-2 \tau}\right)\right] v+\mathrm{O}\left(\left(\sqrt{\mu}+\mathrm{e}^{\tau}\right) \mathrm{e}^{\tau}|v|\right)+\mathrm{O}\left(\mu \mathrm{e}^{-2 \tau}\right) Q_{n}^{0}+\mathrm{O}\left(\sqrt{\mu} \mathrm{e}^{\tau}\left|Q_{n}^{0}\right|\right) \\
& =\left[D+\mathrm{O}\left(\mu \mathrm{e}^{-2 \tau}\right)\right] v+\mathrm{O}\left(\left(\sqrt{\mu}+\mathrm{e}^{\tau}\right) \mathrm{e}^{\tau}|v|\right)+\mathrm{O}\left(\mu \mathrm{e}^{-3 \tau / 2}+\sqrt{\mu} \mathrm{e}^{3 \tau / 2}\right)\left|q_{n}^{0}\right| .
\end{aligned}
$$

We denote by $H(\tau)$ the Heaviside function, which vanishes for $\tau<0$. The equation

$$
v_{\tau}=\left[D+H\left(\tau-\tau_{0}\right) \mathrm{O}\left(\mu \mathrm{e}^{-2 \tau}\right)\right] v
$$

has an exponential dichotomy for $\tau \leq \tau_{1}$, uniformly in $\mu$, since $\mathrm{O}\left(\mu \mathrm{e}^{-2 \tau}\right)=\mathrm{O}\left(1 / r_{0}^{2}\right)$ for $\tau \geq \tau_{0}$ and $D$ is hyperbolic. Choosing coordinates $\tilde{v}$ in the $\tau$-dependent stable and unstable subspaces given by the exponential dichotomy, we obtain the system

$$
\tilde{v}_{\tau}=\left[D+\mathrm{O}\left(\mu \mathrm{e}^{-2 \tau}\right) \mathrm{id}\right] \tilde{v}+\mathrm{O}\left(\left(\sqrt{\mu}+\mathrm{e}^{\tau}\right) \mathrm{e}^{\tau}|\tilde{v}|\right)+\mathrm{O}\left(\mu \mathrm{e}^{-3 \tau / 2}+\sqrt{\mu} \mathrm{e}^{3 \tau / 2}\right)\left|q_{n}^{0}\right|,
$$

where id is the identity matrix. The linear transformation $v \mapsto \tilde{v}$ is $\mathrm{O}\left(1 / r_{0}^{2}\right)$-close to the identity for $\tau \in\left[\tau_{0}, \tau_{1}\right]$, and, since the unstable subspace is unique in backward time, we also have

$$
v\left(\tau_{0}\right)=\left(\begin{array}{cc}
1 & \mathrm{O}\left(r_{0}^{-2}\right) \\
0 & 1
\end{array}\right) \tilde{v}\left(\tau_{0}\right),
$$

which we shall exploit later on. It is straightforward to check that the solution operator $\Phi(\tau, \sigma)$ of

$$
\tilde{v}_{\tau}=\left[D+\mathrm{O}\left(\mu \mathrm{e}^{-2 \tau}\right) \mathrm{id}\right] \tilde{v}
$$

is given by

$$
\Phi(\tau, \sigma)=\left(\begin{array}{cc}
\mathrm{e}^{(\tau-\sigma) / 2}\left[1+\mathrm{O}\left(1 / r_{0}^{2}\right)\right] & 0 \\
0 & \mathrm{e}^{-(\tau-\sigma) / 2}\left[1+\mathrm{O}\left(1 / r_{0}^{2}\right)\right]
\end{array}\right)
$$

uniformly for $\tau, \sigma \in\left[\tau_{0}, \tau_{1}\right]$. We can therefore rewrite (4.14) as the integral equation

$$
\begin{aligned}
& \tilde{v}_{1}(\tau)=\int_{\tau_{0}}^{\tau} \mathrm{e}^{\frac{1}{2}(\tau-\sigma)} \mathrm{O}\left(\left(\sqrt{\mu}+\mathrm{e}^{\sigma}\right) \mathrm{e}^{\sigma}|\tilde{v}(\sigma)|\right) \mathrm{d} \sigma+\int_{\tau_{1}}^{\tau} \mathrm{e}^{\frac{1}{2}(\tau-\sigma)} \mathrm{O}\left(\mu \mathrm{e}^{-3 \sigma / 2}+\sqrt{\mu} \mathrm{e}^{3 \sigma / 2}\right)\left|q_{n}^{0}\right| \mathrm{d} \sigma \\
& \tilde{v}_{2}(\tau)=\int_{\tau_{0}}^{\tau} \mathrm{e}^{-\frac{1}{2}(\tau-\sigma)} \mathrm{O}\left(\left(\sqrt{\mu}+\mathrm{e}^{\sigma}\right) \mathrm{e}^{\sigma}|\tilde{v}(\sigma)|\right) \mathrm{d} \sigma+\int_{\tau_{0}}^{\tau} \mathrm{e}^{-\frac{1}{2}(\tau-\sigma)} \mathrm{O}\left(\mu \mathrm{e}^{-3 \sigma / 2}+\sqrt{\mu} \mathrm{e}^{3 \sigma / 2}\right)\left|q_{n}^{0}\right| \mathrm{d} \sigma
\end{aligned}
$$

on $\left[\tau_{0}, \tau_{1}\right]$. We find that the right-hand side is a contraction in $\tilde{v}$ with Lipschitz constant $C s_{1}^{2} \ll 1$ in $C^{0}\left(\left[\tau_{0}, \tau_{1}\right]\right)$. Furthermore, the inhomogeneous terms are bounded by

$$
\left|q_{n}^{0}\right|\left[\mathrm{O}\left(\mu^{\frac{1}{4}} r_{0}^{-\frac{3}{2}}\right)+\mathrm{O}_{r_{0}}(\sqrt{\mu})\right]
$$

uniformly in $\left[\tau_{0}, \tau_{1}\right]$. Thus, (4.18) has a unique solution $\tilde{v}=\tilde{V}_{n}(\tau)$, which is bounded in the $L^{\infty}$-norm by (4.19) and depends continuously on $\mu$ (when rescaled to a common interval of definition). Transforming this solution back to the $v$-variables, we find that $Q_{n}=Q_{n}^{0}+V_{n}$ satisfies $(4.10)$ on $\left[\tau_{0}, \tau_{1}\right]$ for all sufficiently small $\mu \geq 0$. Furthermore, using (4.11), (4.15) and the bound (4.19) on $\tilde{V}_{n}$, we have

$$
Q_{n}\left(\tau_{0}\right)=q_{n}^{0} \mu^{\frac{1}{4}} r_{0}^{\frac{1}{2}}\left[1+\mathrm{O}\left(r_{0}^{-2}\right)\right]\left(\begin{array}{l}
1 \\
0
\end{array}\right)+\mathrm{O}_{r_{0}}(\mu)\left|q_{n}^{0}\right|, \quad Q_{n}\left(\tau_{1}\right)=Q_{n}^{0}\left(\tau_{1}\right)+\mathrm{O}\left(\mu^{\frac{1}{4}} r_{0}^{-\frac{3}{2}}\right)+\mathrm{O}_{r_{0}}(\sqrt{\mu}) .
$$


In the next step of our analysis, we consider the full equation (4.10) with $u=Q_{n}+v$ near the solution $Q_{n}$ we just constructed. Since $Q_{n}$ satisfies (4.10) for all $\mu \geq 0$, the equation for $v$ is of the form

$$
v_{\tau}=\left[D+\mathrm{O}\left(\mu \mathrm{e}^{-2 \tau}\right)\right] v+\mathrm{O}\left(\left(\sqrt{\mu}+\mathrm{e}^{\tau}\right) \mathrm{e}^{\tau}|v|\right) .
$$

We can again use the variables $\tilde{v}$ that correspond to the exponential dichotomies of the linear part given in (4.13). The corresponding equation is given by

$$
\tilde{v}_{\tau}=\left[D+\mathrm{O}\left(\mu \mathrm{e}^{-2 \tau}\right) \mathrm{id}\right] \tilde{v}+\mathrm{O}\left(\left(\sqrt{\mu}+\mathrm{e}^{\tau}\right) \mathrm{e}^{\tau}|\tilde{v}|\right) .
$$

We now solve this equation on $\left[\tau_{0}, \tau_{1}\right]$ by reformulating it as the fixed-point equation

$$
\begin{aligned}
& \tilde{v}_{1}(\tau)=\mathrm{e}^{\frac{1}{2}\left(\tau+\tau_{0}-\tau_{1}\right)}\left[1+\mathrm{O}\left(r_{0}^{-2}\right)\right] \tilde{v}_{1}^{0}+\int_{\tau_{1}}^{\tau} \mathrm{e}^{\frac{1}{2}(\tau-\sigma)} \mathrm{O}\left(\left(\sqrt{\mu}+\mathrm{e}^{\sigma}\right) \mathrm{e}^{\sigma}|\tilde{v}(\sigma)|\right) \mathrm{d} \sigma=: \mathcal{G}_{1}\left(\tilde{v}^{0}, \tilde{v}, \mu\right)(\tau) \\
& \tilde{v}_{2}(\tau)=\mathrm{e}^{-\frac{1}{2}\left(\tau-\tau_{0}\right)}\left[1+\mathrm{O}\left(r_{0}^{-2}\right)\right] \tilde{v}_{2}^{0}+\int_{\tau_{0}}^{\tau} \mathrm{e}^{-\frac{1}{2}(\tau-\sigma)} \mathrm{O}\left(\left(\sqrt{\mu}+\mathrm{e}^{\sigma}\right) \mathrm{e}^{\sigma}|\tilde{v}(\sigma)|\right) \mathrm{d} \sigma=: \mathcal{G}_{2}\left(\tilde{v}^{0}, \tilde{v}, \mu\right)(\tau),
\end{aligned}
$$

where $\tilde{v}^{0}=\left(\tilde{v}_{1}^{0}, \tilde{v}_{2}^{0}\right) \in \mathbb{C}^{2}$. The reason for seeking $\tilde{v}_{1}\left(\tau_{1}\right)$ in the form $\tilde{v}_{1}\left(\tau_{1}\right)=\mathrm{e}^{\tau_{0} / 2} \tilde{v}_{1}^{0}$ will become clear below. To solve (4.23), we use the norms

$$
\left\|\tilde{v}_{1}\right\|_{\mathrm{u}}:=\mathrm{e}^{-\tau_{0} / 2} \sup _{\tau \in\left[\tau_{0}, \tau_{1}\right]}\left|\tilde{v}_{1}(\tau)\right|, \quad\left\|\tilde{v}_{2}\right\|_{\mathrm{s}}:=\sup _{\tau \in\left[\tau_{0}, \tau_{1}\right]} \mathrm{e}^{\frac{1}{2}\left(\tau-\tau_{0}\right)}\left|\tilde{v}_{2}(\tau)\right|, \quad\|\tilde{v}\|_{\mathrm{h}}:=\left\|\tilde{v}_{1}\right\|_{\mathrm{u}}+\left\|\tilde{v}_{2}\right\|_{\mathrm{s}}
$$

for $\tilde{v}$ and its components and obtain the estimates

$$
\begin{aligned}
\left\|\mathcal{G}_{1}\left(\tilde{v}^{0}, \tilde{v}, \mu\right)\right\|_{\mathrm{u}} & \leq C\left[\left|\tilde{v}_{1}^{0}\right|+\left(\mathrm{e}^{\tau_{1}}+\sqrt{\mu}\right)\|\tilde{v}\|_{\mathrm{h}}\right] \\
\left\|\mathcal{G}_{2}\left(\tilde{v}^{0}, \tilde{v}, \mu\right)\right\|_{\mathrm{s}} & \leq C\left[\left|\tilde{v}_{2}^{0}\right|+\mathrm{e}^{\tau_{1}}\left(\mathrm{e}^{\tau_{1}}+\sqrt{\mu}\right)\|\tilde{v}\|_{\mathrm{h}}\right]
\end{aligned}
$$

for each $\tilde{v} \in C^{0}\left(\left[\tau_{0}, \tau_{1}\right], \mathbb{C}^{2}\right)$, where the constant $C$ does not depend on $\left(\tilde{v}^{0}, \tilde{v}, \tau_{0}, \tau_{1}, \mu\right)$. Thus, upon choosing $0<s_{1}=\mathrm{e}^{\tau_{1}} \ll 1$ sufficiently small, we can apply the uniform contraction mapping principle to (4.23): there exists a $\delta>0$ so that (4.23) has a unique solution $\tilde{v} \in C^{0}\left(\left[\tau_{0}, \tau_{1}\right], \mathbb{C}^{2}\right)$ for all $\tilde{v}^{0}$ and $\mu$ with $\left|\tilde{v}^{0}\right|<\delta$ and $0<\mu<\delta$. Furthermore, the solution depends smoothly on $\tilde{v}^{0}$ and continuously on $\mu$ (when rescaled to a common interval of definition) and satisfies the estimate

$$
\|\tilde{v}\|_{\mathrm{h}} \leq C\left|\tilde{v}^{0}\right|
$$

for some constant $C$ that is independent of $\left(\tilde{v}^{0}, \tau_{0}, \tau_{1}, \mu\right)$. Below, we shall need $\tilde{v}\left(\tau_{1}\right)$ and $\tilde{v}\left(\tau_{0}\right)$, which are given by

$$
\tilde{v}_{1}\left(\tau_{1}\right)=\mathrm{e}^{\tau_{0} / 2} \tilde{v}_{1}^{0}, \quad \tilde{v}_{2}\left(\tau_{1}\right)=\mathrm{e}^{\left(\tau_{0}-\tau_{1}\right) / 2}\left[1+\mathrm{O}\left(r_{0}^{-2}\right)\right] \tilde{v}_{2}^{0}+\mathrm{e}^{\tau_{0} / 2} \mathrm{O}\left(\left(\mathrm{e}^{\tau_{1}}+\sqrt{\mu}\right)\left|\tilde{v}^{0}\right|\right)
$$

and

$$
\begin{aligned}
\tilde{v}_{1}\left(\tau_{0}\right) & =\mathrm{e}^{\tau_{0}-\tau_{1} / 2}\left[1+\mathrm{O}\left(r_{0}^{-2}\right)\right] \tilde{v}_{1}^{0}+\int_{\tau_{1}}^{\tau_{0}} \mathrm{e}^{\frac{1}{2}\left(\tau_{0}-\sigma\right)} \mathrm{e}^{\sigma} \mathrm{O}\left(\left(\mathrm{e}^{\sigma}+\sqrt{\mu}\right)|\tilde{v}(\sigma)|\right) \mathrm{d} \sigma \\
& =\mathrm{e}^{\tau_{0}}\left(\mathrm{e}^{-\tau_{1} / 2}\left[1+\mathrm{O}\left(r_{0}^{-2}\right)\right] \tilde{v}_{1}^{0}+\mathrm{O}\left(\mathrm{e}^{\tau_{1}}\left|\tilde{v}^{0}\right|\right)\right) \\
\tilde{v}_{2}\left(\tau_{0}\right) & =\tilde{v}_{2}^{0},
\end{aligned}
$$

where we used (4.25) and (4.26). Note that the terms $\mathrm{O}\left(r_{0}^{-2}\right)$ are not present when we evaluate $\Phi(\tau, \sigma)$ from $(4.17)$ at $\sigma=\tau$ since $\Phi(\tau, \tau)=$ id.

In the last step of the proof, we need to find all initial data $\tilde{v}^{0}$ for which the corresponding solution $Q_{n}\left(\tau_{1}\right)+v\left(\tau_{1}\right)$, in the original $v$-coordinates, lies in the $s=s_{1}$ fiber of $W_{+}^{\mathrm{s}}(\mu)$. Once this is achieved, $Q_{n}\left(\tau_{0}\right)+v\left(\tau_{0}\right)$ lies in the $s=\sqrt{\mu} r_{0}$ fiber of $W_{+}^{\mathrm{s}}(\mu)$, as desired. Thus, we set $\tau=\tau_{1}$. The transversality inside the real subspace of the stable manifold $W_{+}^{\mathrm{s}}(0)$ and the unstable manifold $W_{-}^{\mathrm{u}}(0)$ of $(4.22)$ for $\mu=0$ implies that $u=Q_{n}^{0}\left(\tau_{1}\right)+v\left(\tau_{1}\right)$ lies in the $\tau=\tau_{1}$ fiber of the stable manifold $W_{+}^{\mathrm{s}}(\mu)$ for any sufficiently small $\mu$ if and only if

$$
v_{1}\left(\tau_{1}\right)=g\left(v_{2}\left(\tau_{1}\right), \mu\right), \quad\left|g\left(v_{2}, \mu\right)\right| \leq C\left(\left|v_{2}\right|+|\mu|\right)
$$


for an appropriate smooth function $g$, where $C$ is some constant that is independent of $\tau_{1}$ and $\mu$ : the estimate for $g$ follows from (4.1) and the fact that $\tau_{1}$ is fixed independently of $\mu$. Since the transformation $v \mapsto \tilde{v}$ is $\mathrm{O}\left(1 / r_{0}^{2}\right)$-close to the identity, we have a similar characterization of the stable manifold that uses $\tilde{v}\left(\tau_{1}\right)$ :

$$
u\left(\tau_{1}\right)=Q_{n}\left(\tau_{1}\right)+v\left(\tau_{1}\right) \stackrel{(4.20)}{=} Q_{n}^{0}\left(\tau_{1}\right)+\tilde{v}\left(\tau_{1}\right)+\mathrm{O}\left(\mu^{\frac{1}{4}} r_{0}^{-\frac{3}{2}}\right)+\mathrm{O}_{r_{0}}(\sqrt{\mu})
$$

lies in the stable manifold $W_{+}^{\text {s }}(\mu)$ if and only if

$$
\tilde{v}_{1}\left(\tau_{1}\right)+\mathrm{O}\left(\mu^{\frac{1}{4}} r_{0}^{-\frac{3}{2}}\right)+\mathrm{O}_{r_{0}}(\sqrt{\mu})=\tilde{g}\left(\tilde{v}_{2}\left(\tau_{1}\right)+\mathrm{O}\left(\mu^{\frac{1}{4}} r_{0}^{-\frac{3}{2}}\right)+\mathrm{O}_{r_{0}}(\sqrt{\mu}), \mu\right), \quad\left|\tilde{g}\left(v_{2}, \mu\right)\right| \leq C\left(\left|v_{2}\right|+|\mu|\right) .
$$

We need to choose $\tilde{v}^{0}$ so that (4.29) is met. Substituting the expression (4.27) for $\tilde{v}\left(\tau_{1}\right)$ and using that $\mathrm{e}^{\tau_{0} / 2}=\mu^{\frac{1}{4}} \sqrt{r_{0}}$, (4.29) becomes

$$
\mu^{\frac{1}{4}} \sqrt{r_{0}}\left[\tilde{v}_{1}^{0}+\mathrm{O}\left(r_{0}^{-2}\right)+\mathrm{O}_{r_{0}}\left(\mu^{\frac{1}{4}}\right)\right]=\tilde{g}\left(\mu^{\frac{1}{4}} \sqrt{r_{0}}\left[s_{1}^{-\frac{1}{2}}\left[1+\mathrm{O}\left(r_{0}^{-2}\right)\right] \tilde{v}_{2}^{0}+\mathrm{O}\left(\left(s_{1}+\sqrt{\mu}\right)\left|\tilde{v}^{0}\right|\right)+\mathrm{O}\left(r_{0}^{-2}\right)+\mathrm{O}_{r_{0}}\left(\mu^{\frac{1}{4}}\right)\right], \mu\right) .
$$

Using the estimate for $\tilde{g}$ from (4.29), this equation is equivalent to

$$
\tilde{v}_{1}^{0}=\mathrm{O}\left(s_{1}^{-\frac{1}{2}} \tilde{v}_{2}^{0}\right)+\mathrm{O}\left(\left(s_{1}+\sqrt{\mu}\right)\left|\tilde{v}^{0}\right|\right)+\mathrm{O}\left(r_{0}^{-2}\right)+\mathrm{O}_{r_{0}}\left(\mu^{\frac{1}{4}}\right) .
$$

Thus, choosing $0<s_{1} \ll 1$ sufficiently small, we can solve uniquely for $\tilde{v}_{1}^{0}$ as a function of $\left(\tilde{v}_{2}^{0}, \mu\right)$ with

$$
\tilde{v}_{1}^{0}=\mathrm{O}\left(s_{1}^{-\frac{1}{2}} \tilde{v}_{2}^{0}+r_{0}^{-2}\right)+\mathrm{O}_{r_{0}}\left(\mu^{\frac{1}{4}}\right) .
$$

To obtain the desired expression for $W_{+}^{\mathrm{s}}(\mu)$ at $s=\sqrt{\mu} r_{0}$, we substitute (4.30) into the expression (4.28) for $\tilde{v}\left(\tau_{0}\right)$ and obtain

$$
\begin{aligned}
\tilde{v}_{1}\left(\tau_{0}\right) & =\sqrt{\mu} r_{0}\left(s_{1}^{-\frac{1}{2}}\left[1+\mathrm{O}\left(r_{0}^{-2}\right)\right] \tilde{v}_{1}^{0}+\mathrm{O}\left(s_{1}\left|\tilde{v}^{0}\right|\right)\right) \\
& =\sqrt{\mu} r_{0} \mathrm{O}\left(s_{1}^{-1} \tilde{v}_{2}^{0}+r_{0}^{-2} s_{1}^{-\frac{1}{2}}\right)+\mathrm{O}_{r_{0}}\left(\mu^{\frac{3}{4}}\right) \\
\tilde{v}_{2}\left(\tau_{0}\right) & =\tilde{v}_{2}^{0} .
\end{aligned}
$$

Using the transformation (4.15) to transform from $\tilde{v}$ back to the $v$-variable and adding the solution $Q_{n}\left(\tau_{0}\right)$ from (4.20), we find that the $\tau=\tau_{0}$ fiber of $W_{+}^{\mathrm{s}}(\mu)$ can be parametrized by

$$
u\left(\tau_{0}\right)=Q_{n}\left(\tau_{0}\right)+v\left(\tau_{0}\right)=\left(\begin{array}{c}
q_{n}^{0} \mu^{\frac{1}{4}} r_{0}^{\frac{1}{2}}\left[1+\mathrm{O}\left(r_{0}^{-2}\right)\right]+\mathrm{O}\left(\sqrt{\mu} r_{0}^{-1} s_{1}^{-\frac{1}{2}}\right)+\mathrm{O}\left(r_{0}^{-2} \tilde{v}_{2}^{0}\right)+\mathrm{O}_{r_{0}}(\mu) \\
\tilde{v}_{2}^{0}+\mathrm{O}_{r_{0}}(\mu)
\end{array}\right) .
$$

Repeating this analysis near the rotated orbits $\mathrm{e}^{\mathrm{i} \eta_{2}} Q_{n}^{0}$, writing $\tilde{v}_{2}^{0}=\eta_{1} \mathrm{e}^{\mathrm{i} \eta_{2}}$, and transforming the resulting expression back to the $(\tilde{a}, \tilde{b})$ coordinates, we arrive at $(4.7)$.

Recall that $a=\tilde{a}$ and $b=(\tilde{b}+\tilde{a} / 2) / s$; see (3.17). For $s=\sqrt{\mu} r_{0}$, a vector of the form

$$
u=c_{1}\left(\begin{array}{l}
1 \\
0
\end{array}\right)+c_{2}\left(\begin{array}{l}
0 \\
1
\end{array}\right) \Longleftrightarrow\left(\begin{array}{c}
\tilde{a} \\
\tilde{b}
\end{array}\right)=c_{1}\left(\begin{array}{c}
1 \\
\frac{1}{2}
\end{array}\right)+c_{2}\left(\begin{array}{r}
1 \\
-\frac{1}{2}
\end{array}\right)
$$

therefore becomes

$$
\left(\begin{array}{l}
a \\
b
\end{array}\right)=c_{1}\left(\begin{array}{c}
1 \\
\frac{1}{\sqrt{\mu} r_{0}}
\end{array}\right)+c_{2}\left(\begin{array}{l}
1 \\
0
\end{array}\right)
$$

and, since $A=\sqrt{\mu} a$ and $B=\mu b$, we get

$$
\left(\begin{array}{c}
A \\
B
\end{array}\right)=\sqrt{\mu}\left[c_{1}\left(\begin{array}{c}
1 \\
\frac{1}{r_{0}}
\end{array}\right)+c_{2}\left(\begin{array}{c}
1 \\
0
\end{array}\right)\right] .
$$


Thus, the $s=\sqrt{\mu} r_{0}$ fiber of the stable manifold given by

$$
\left.W_{+}^{\mathrm{s}}(\mu)\right|_{r=r_{0}}:\left(\begin{array}{c}
\tilde{a} \\
\tilde{b}
\end{array}\right)=\mathrm{e}^{\mathrm{i} \eta_{2}}\left[\left(\mu^{\frac{1}{4}} r_{0}^{\frac{1}{2}} q_{n}^{0}\left[1+\mathrm{O}\left(r_{0}^{-2}\right)\right]+\mathrm{O}\left(r_{0}^{-2} \eta_{1}\right)+\mathrm{O}_{r_{0}}\left(\mu^{\frac{1}{2}}\right)\right)\left(\begin{array}{c}
1 \\
\frac{1}{2}
\end{array}\right)+\left(\eta_{1}+\mathrm{O}_{r_{0}}(\mu)\right)\left(\begin{array}{c}
1 \\
-\frac{1}{2}
\end{array}\right)\right]
$$

becomes

$$
\left.W_{+}^{\mathrm{s}}(\mu)\right|_{r=r_{0}}:\left(\begin{array}{c}
A \\
B
\end{array}\right)=\sqrt{\mu} \mathrm{e}^{\mathrm{i} \eta_{2}}\left[\left(\mu^{\frac{1}{4}} r_{0}^{\frac{1}{2}} q_{n}^{0}\left[1+\mathrm{O}\left(r_{0}^{-2}\right)\right]+\mathrm{O}\left(r_{0}^{-2} \eta_{1}\right)+\mathrm{O}_{r_{0}}\left(\mu^{\frac{1}{2}}\right)\right)\left(\begin{array}{c}
1 \\
\frac{1}{r_{0}}
\end{array}\right)+\left(\eta_{1}+\mathrm{O}_{r_{0}}(\mu)\right)\left(\begin{array}{c}
1 \\
0
\end{array}\right)\right] .
$$

in the $(A, B)$ coordinates.

Our goal is to find intersections of the stable far-field manifold $W_{+}^{\mathrm{s}}(\mu)$ and the core manifold $W_{-}^{\text {cu }}(\mu)$, given by $(3.23)$

$$
\left.W_{-}^{\mathrm{cu}}(\mu)\right|_{r=r_{0}}:\left(\begin{array}{c}
A \\
B
\end{array}\right)=\mathrm{e}^{\mathrm{i}\left[-\pi / 4+\mathrm{O}\left(1 / r_{0}^{2}\right)+\mathrm{O}_{r_{0}}\left(|\mu|+|d|^{2}\right)\right]}\left(\begin{array}{c}
d_{1}-\mathrm{i} d_{2}+\mathrm{O}\left(\frac{1}{r_{0}}\right) d+\mathrm{O}_{r_{0}}\left(|\mu||d|+|d|^{2}\right) \\
-\frac{\mathrm{i} d_{2}}{r_{0}}+\mathrm{O}\left(\frac{1}{r_{0}^{2}}\right) d_{2}+\mathrm{O}_{r_{0}}\left(|\mu||d|+|d|^{2}\right)
\end{array}\right),
$$

for small $d \in \mathbb{R}^{2}$. Upon redefining $\eta_{2}$, we therefore need to solve

$$
\begin{aligned}
& \left(\begin{array}{c}
d_{1}-\mathrm{i} d_{2} \\
-\mathrm{i} d_{2}
\end{array}\right)+\mathrm{O}\left(\frac{|d|}{r_{0}}\right)+\mathrm{O}_{r_{0}}\left(\mu^{\frac{3}{2}}+|d|^{2}\right) \\
& =\sqrt{\mu} \mathrm{e}^{\mathrm{i} \eta_{2}}\left[\left(\mu^{\frac{1}{4}} r_{0}^{\frac{1}{2}} q_{n}^{0}\left[1+\mathrm{O}\left(r_{0}^{-2}\right)\right]+\mathrm{O}\left(r_{0}^{-2} \eta_{1}\right)+\mathrm{O}_{r_{0}}\left(\mu^{\frac{1}{2}}\right)\right)\left(\begin{array}{c}
1 \\
1
\end{array}\right)+\left(\begin{array}{c}
\eta_{1} \\
0
\end{array}\right)\right] .
\end{aligned}
$$

Writing $d_{j}=\sqrt{\mu} \hat{d}_{j}$, the above equation becomes

$$
\begin{aligned}
& \left(\begin{array}{c}
\hat{d}_{1}-\mathrm{i} \hat{d}_{2} \\
-\mathrm{i} \hat{d}_{2}
\end{array}\right)+\mathrm{O}\left(\frac{|\hat{d}|}{r_{0}}\right)+\mathrm{O}_{r_{0}}\left(\mu+\sqrt{\mu}|\hat{d}|^{2}\right) \\
& =\mathrm{e}^{\mathrm{i} \eta_{2}}\left[\left(\mu^{\frac{1}{4}} r_{0}^{\frac{1}{2}} q_{n}^{0}\left[1+\mathrm{O}\left(r_{0}^{-2}\right)\right]+\mathrm{O}\left(r_{0}^{-2} \eta_{1}\right)+\mathrm{O}_{r_{0}}\left(\mu^{\frac{1}{2}}\right)\right)\left(\begin{array}{c}
1 \\
1
\end{array}\right)+\left(\begin{array}{c}
\eta_{1} \\
0
\end{array}\right)\right]
\end{aligned}
$$

We set $\eta_{2}=\hat{\eta}_{2} \pm \pi / 2$ and reorder terms to arrive at

$$
\left(\begin{array}{c}
\hat{d}_{1}-\mathrm{i} \hat{d}_{2}-\mathrm{i} \eta_{1} \mathrm{e}^{\mathrm{i} \hat{\eta}_{2}} \\
-\mathrm{i} \hat{d}_{2}
\end{array}\right)+\mathrm{O}\left(\frac{|\hat{d}|}{r_{0}}\right)= \pm \mu^{\frac{1}{4}} \mathrm{e}^{\mathrm{i} \hat{\eta}_{2}}\left(\sqrt{r_{0}} q_{n}^{0}\left[1+\mathrm{O}\left(r_{0}^{-2}\right)\right]+\mathrm{O}\left(r_{0}^{-2}\left|\eta_{1}\right|\right)\right)\left(\begin{array}{c}
\mathrm{i} \\
\mathrm{i}
\end{array}\right)+\sqrt{\mu} \mathrm{O}_{r_{0}}\left(1+|\hat{d}|^{2}\right) .
$$

For either sign, we can solve the $(\operatorname{Re} A, \operatorname{Im} A, \operatorname{Im} B)$-components of $(4.34)$ uniquely for $\left(\hat{d}_{1}, \hat{d}_{2}, \eta_{1}\right)$ as functions of $\left(\hat{\eta}_{2}, \mu\right)$ and obtain the estimate

$$
\left(\hat{d}_{1}, \hat{d}_{2}, \eta_{1}\right)=\mathrm{O}\left(\sqrt{r_{0}} \mu^{\frac{1}{4}}\right)+\mathrm{O}_{r_{0}}(\sqrt{\mu})
$$

for this solution. The $(\operatorname{Re} B)$-component of (4.34) is then given by

$$
\sqrt{r_{0}} q_{n}^{0} \hat{\eta}_{2}\left(1+\mathrm{O}\left(\hat{\eta}_{2}^{2}\right)\right)=\mathrm{O}\left(\frac{1}{\sqrt{r_{0}}}\right)+\mathrm{O}_{r_{0}}\left(\mu^{\frac{1}{4}}\right) .
$$

Choosing $r_{0} \gg 1$ sufficiently large, we can solve (4.35) uniquely for $\hat{\eta}_{2}$ as a function of $\mu$ since $q_{n}^{0} \neq 0$, which proves the existence of pairs of ring solutions.

For the original amplitudes from (2.7) given by

$$
\left(\tilde{d}_{1}, \tilde{d}_{2}\right) \stackrel{(3.21)}{=}\left(\sqrt{r_{0}} d_{1}, d_{2} / \sqrt{r_{0}}\right)=\mu^{\frac{1}{2}}\left(\sqrt{r_{0}} \hat{d}_{1}, \hat{d}_{2} / \sqrt{r_{0}}\right),
$$

we obtain the estimate $\left(\tilde{d}_{1}, \tilde{d}_{2}\right)=\mathrm{O}\left(\mu^{\frac{3}{4}}\right)$ as claimed in Theorem 1. This result can be improved as follows. The terms $\mathrm{O}\left(1 / r_{0}\right) \hat{d}$ can be calculated to leading order using the expression (3.11) together with expansions of the Bessel functions that appear in (2.4). A more refined analysis of (4.34), which we omit, then gives the expansion

$$
\left(\tilde{d}_{1}, \tilde{d}_{2}\right)= \pm q_{n}^{0} \mu^{\frac{3}{4}}\left(\frac{1}{2},-1\right)+\mathrm{O}(\mu)
$$


so that the radial ring solutions are given approximately by

$$
u(r)= \pm q_{n}^{0} \mu^{\frac{3}{4}}\left[\frac{1}{2} J_{0}(r)-r J_{1}(r)\right]+\mathrm{O}(\mu)
$$

uniformly for $r$ in bounded intervals. In the far field, the amplitude of the radial rings $u(r)$ scales with $\sqrt{\mu}$, and the expansion of $u(r)$ in terms of the envelope pulses $q_{n}(s)$ that we stated in Theorem 1 follows from our proof once we reverse the coordinate transformation (3.6). This completes the proof of Theorem 1.

\section{$5 \quad$ Planar spot solutions for $\nu \neq 0$}

As outlined in $\S 1$, spots arise since the tangent spaces $T_{0} W_{-}^{\mathrm{cu}}(\mu)$ and $T_{0} W_{+}^{\mathrm{s}}(\mu)$ of the core manifold and the stable manifold at $u=0$ have a one-dimensional intersection when $\mu=0$. If this tangency is quadratic, then spots will bifurcate for $\mu>0$ since the core manifold and stable manifold intersect transversely along $u=0$ for $\mu>0$ as $u=0$ is then a temporally stable solution.

Formally, we can find intersections of these tangent spaces by inspecting the solutions $V_{j}(r)$ of the linearization of (2.2) about $u=0$ at $\mu=0$, which are given in (2.4). We find that $V_{1}(r)$ and $V_{3}(r)$ decay like $1 / \sqrt{r}$, while $V_{2}(r)$ and $V_{4}(r)$ grow like $\sqrt{r}$ as $r \rightarrow \infty$. Hence, we may expect that the tangent space $T_{0} W_{+}^{\text {s }}(0)$ of the stable manifold at $(u, \mu)=0$ is spanned by $V_{1}(r)$ and $V_{3}(r)$. On the other hand, we showed in Lemma 1 that the tangent space $T_{0} W_{-}^{\mathrm{cu}}(0)$ is spanned by $V_{1}(r)$ and $V_{2}(r)$. Thus, these tangent spaces would then intersect along the one-dimensional subspace spanned by $V_{1}(r)$. This argument is formal because the decay of the solutions $V_{1}$ and $V_{3}$ is only algebraic in $r$ as $r \rightarrow \infty$.

To make these arguments rigorous, we proceed as for ring solutions. We first construct the stable manifold $W_{+}^{\mathrm{s}}(\mu)$ of $u=0$ near $u=0$ in the coordinates $(\hat{a}, \hat{b})$. Upon transforming the resulting expression into $(\tilde{a}, \tilde{b})$, we can track the stable manifold back to $r=r_{0}$ as in the preceding section. We can then match with the core manifold $W_{-}^{\text {cu }}(\mu)$ to find radial spot solutions.

We start by linearizing the far-field equation $(3.16)$ about $(\hat{a}, \hat{b})=0$ to get the equation

$$
\left(\begin{array}{c}
\hat{a}_{s} \\
\hat{b}_{s}
\end{array}\right)=\left(\begin{array}{cc}
0 & 1 \\
\frac{1}{4} & 0
\end{array}\right)\left(\begin{array}{c}
\hat{a} \\
\hat{b}
\end{array}\right)+\mathrm{O}(\mu)\left(\begin{array}{c}
\hat{a} \\
\hat{b}
\end{array}\right),
$$

where the estimate of the remainder term is valid uniformly in $s_{1} \leq s<\infty$ for each fixed $s_{1}>0$. For $\mu=0$, the general solution of (5.1) is given by

$$
\left(\begin{array}{c}
\hat{a} \\
\hat{b}
\end{array}\right)(s)=c_{1} \mathrm{e}^{-s / 2}\left(\begin{array}{c}
1 \\
-\frac{1}{2}
\end{array}\right)+c_{2} \mathrm{e}^{s / 2}\left(\begin{array}{c}
1 \\
\frac{1}{2}
\end{array}\right) .
$$

Thus, for each fixed $s=s_{1}>0$ and for all sufficiently small $\mu>0$, we can write the $s=s_{1}$-fiber of the stable manifold $W_{+}^{\mathrm{s}}(\mu)$ of $(3.16)$ near $u=0$ as

$$
\left.W_{+}^{\mathrm{s}}(\mu)\right|_{s=s_{1}}:\left(\begin{array}{c}
\hat{a} \\
\hat{b}
\end{array}\right)=\eta\left(\begin{array}{r}
1 \\
-\frac{1}{2}
\end{array}\right)+\mathrm{O}_{s_{1}}\left(|\mu \eta|+|\eta|^{3}\right)\left(\begin{array}{c}
1 \\
\frac{1}{2}
\end{array}\right),
$$

where $\eta \in \mathbb{C}$. Using (3.15) and (3.17) and redefining $\eta$, we obtain the expression

$$
\left.W_{+}^{\mathrm{s}}(\mu)\right|_{s=s_{1}}:\left(\begin{array}{c}
\tilde{a} \\
\tilde{b}
\end{array}\right)=\eta\left(\begin{array}{c}
1 \\
-\frac{1}{2}-\frac{s_{1}}{2}
\end{array}\right)+\mathrm{O}_{s_{1}}\left(|\mu \eta|+|\eta|^{3}\right)\left(\begin{array}{c}
1 \\
-\frac{1}{2}+\frac{s_{1}}{2}
\end{array}\right)
$$

for $W_{+}^{\mathrm{s}}(\mu)$ in the $(\tilde{a}, \tilde{b})$-coordinates. Next, we transform this expression into the $u$-coordinates given in $(4.9)$ and get

$$
\left.W_{+}^{\mathrm{s}}(\mu)\right|_{s=s_{1}}:\left(\begin{array}{l}
u_{1} \\
u_{2}
\end{array}\right)=\eta\left(\begin{array}{c}
-\frac{s_{1}}{2} \\
1+\frac{s_{1}}{2}
\end{array}\right)+\mathrm{O}_{s_{1}}\left(|\mu \eta|+|\eta|^{3}\right)\left(\begin{array}{c}
\frac{s_{1}}{2} \\
1-\frac{s_{1}}{2}
\end{array}\right) .
$$


Our next task is to track the stable manifold from $s=s_{1}$ to $s=s_{0}:=\sqrt{\mu} r_{0}$, which corresponds to $r=r_{0}$. As in $\S 4$, we use the system (4.10) given by

$$
u_{\tau}=\left[D+\mathrm{O}\left(\mu \mathrm{e}^{-2 \tau}\right)\right] u+\mathrm{O}\left(\left(\sqrt{\mu}+\mathrm{e}^{\tau}\right) \mathrm{e}^{\tau}|u|\right), \quad D=\left(\begin{array}{rr}
\frac{1}{2} & 0 \\
0 & -\frac{1}{2}
\end{array}\right),
$$

where $\tau=\log s$ and $\tau_{j}:=\log s_{j}$.

Lemma 6 The linear equation

$$
u_{\tau}=\left[D+\mathrm{O}\left(\mu \mathrm{e}^{-2 \tau}\right)\right] u
$$

has an exponential dichotomy with exponents $\pm \frac{1}{2}$ on $\left[\tau_{0}, \tau_{1}\right]$. Furthermore, the coordinate transformation $u \mapsto \tilde{u}$ that brings (5.3) into the form

$$
\tilde{u}_{\tau}=\left[D+\mathrm{O}\left(\mu \mathrm{e}^{-2 \tau}\right) \mathrm{id}\right] \tilde{u}
$$

can be chosen such that

$$
u\left(\tau_{0}\right)=\tilde{u}\left(\tau_{0}\right), \quad u\left(\tau_{1}\right)=\left(\begin{array}{cc}
1 & \mathrm{O}(\mu) \\
\mathrm{O}\left(r_{0}^{-2}\right) & 1
\end{array}\right) \tilde{u}\left(\tau_{1}\right)
$$

Proof. As in $\S 4$, we multiply the remainder terms in (5.3) by the Heaviside function $H\left(\tau-\tau_{0}\right)$ and obtain

$$
u_{\tau}=\left[D+H\left(\tau-\tau_{0}\right) \mathrm{O}\left(\mu \mathrm{e}^{-2 \tau}\right)\right] u .
$$

This equation admits exponential dichotomies as claimed, since we have $\mathrm{O}\left(\mu \mathrm{e}^{-2 \tau}\right)=\mathrm{O}\left(r_{0}^{-2}\right)$ for $\tau \in\left[\tau_{0}, \tau_{1}\right]$. As in $\S 4$, we also know that the unstable direction at $\tau=\tau_{0}$ coincides with the $u_{1}$-direction and that the transformation at $\tau=\tau_{1}$ is $\mathrm{O}\left(r_{0}^{-2}\right)$-close to the identity.

It remains to prove that we can choose the stable subspace at $\tau=\tau_{1}$ so that it coincides with the $u_{2}$-direction at $\tau=\tau_{0}$. To this end, we recall that the linearization of the original equation (3.7) about $(A, B)=0$ is, for $\mu=0$, given by

$$
A_{r}=-\frac{\alpha}{2} A+B+\mathrm{O}\left(\alpha^{3}\right) A+\mathrm{O}\left(\alpha^{2}\right) B, \quad B_{r}=-\frac{\alpha}{2} B+\mathrm{O}\left(\alpha^{3}\right) B .
$$

When we transform this equation into the $u$-coordinates, we arrive precisely at (5.3); see the remark after Lemma 3. Going through the coordinate transformations in $\S 3$, it can also be checked that the subspace $(\tilde{A}, \tilde{B})=(1,0)$ becomes the subspace $u=(0,1)$ at $\tau=\tau_{0}$. Note the subspace $(\tilde{A}, \tilde{B})=(1,0)$ is spanned by the solutions $\left(\tilde{A}_{1}, \tilde{B}_{1}\right)$ and $\left(\tilde{A}_{3}, \tilde{B}_{3}\right)$ from (3.20). Transforming the expansions for these solutions into the $(\tilde{a}, \tilde{b})$-coordinates, we obtain the subspace

$$
\left(\begin{array}{c}
\tilde{a} \\
\tilde{b}
\end{array}\right)\left(s_{1}\right)=\left(\begin{array}{c}
1 \\
-\frac{1}{2}+\mathrm{O}\left(\frac{\mu}{s_{1}^{2}}\right)
\end{array}\right)
$$

and, consequently,

$$
u\left(s_{1}\right)=\left(\begin{array}{c}
\mathrm{O}(\mu) \\
1
\end{array}\right)
$$

in the $u$-coordinates. We can now choose this subspace as the stable subspace at $s=s_{1}$, which establishes (5.4) and thus the lemma.

Proceeding as in $\S 4$, we can solve (5.2) formulated in the $\tilde{u}$-variables using the weighted norms (4.24) and obtain a unique solution $\tilde{u}(\tau)$ for each initial data $\tilde{u}^{0}$ with

$$
\tilde{u}_{1}\left(\tau_{1}\right)=\mathrm{e}^{\tau_{0} / 2} \tilde{u}_{1}^{0}, \quad \tilde{u}_{2}\left(\tau_{1}\right)=\mathrm{e}^{\tau_{0} / 2}\left(s_{1}^{-\frac{1}{2}}\left[1+\mathrm{O}\left(r_{0}^{-2}\right)\right] \tilde{u}_{2}^{0}+\mathrm{O}\left(\left(s_{1}+\sqrt{\mu}\right)\left|\tilde{u}^{0}\right|\right)\right)
$$

and

$$
\tilde{u}_{1}\left(\tau_{0}\right)=\mathrm{e}^{\tau_{0}}\left(\mathrm{e}^{-\tau_{1} / 2}\left[1+\mathrm{O}\left(r_{0}^{-2}\right)\right] \tilde{u}_{1}^{0}+\mathrm{O}\left(\mathrm{e}^{\tau_{1}}\left|\tilde{u}^{0}\right|\right)\right), \quad \tilde{u}_{2}\left(\tau_{0}\right)=\tilde{u}_{2}^{0},
$$


see (4.27) and (4.28).

Recall the expression

$$
\left.W_{+}^{\mathrm{s}}(\mu)\right|_{s=s_{1}}: u=\eta\left(\begin{array}{c}
-\frac{s_{1}}{2} \\
1+\frac{s_{1}}{2}
\end{array}\right)+\mathrm{O}_{s_{1}}\left(|\mu \eta|+|\eta|^{3}\right)\left(\begin{array}{c}
\frac{s_{1}}{2} \\
1-\frac{s_{1}}{2}
\end{array}\right)
$$

for the stable manifold in the $u$-variables, which, thanks to (5.4), becomes

$$
\left.W_{+}^{\mathrm{s}}(\mu)\right|_{s=s_{1}}: \tilde{u}=\eta\left(\begin{array}{c}
-\frac{s_{1}}{2}+\mathrm{O}_{s_{1}}(\mu) \\
1+\frac{s_{1}}{2}\left[1+\mathrm{O}\left(r_{0}^{-2}\right)\right]
\end{array}\right)+\mathrm{O}_{s_{1}}\left(|\mu \eta|+|\eta|^{3}\right)\left(\begin{array}{c}
\frac{s_{1}}{2}+\mathrm{O}_{s_{1}}(\mu) \\
1-\frac{s_{1}}{2}\left[1+\mathrm{O}\left(r_{0}^{-2}\right)\right]
\end{array}\right)
$$

in the $\tilde{u}$-variables. Choosing $0<s_{1} \ll 1$ sufficiently small, we can solve

$$
\eta\left(1+\frac{s_{1}}{2}\left[1+\mathrm{O}\left(r_{0}^{-2}\right)\right]\right)+\mathrm{O}_{s_{1}}\left(|\mu \eta|+|\eta|^{3}\right)\left(1-\frac{s_{1}}{2}\left[1+\mathrm{O}\left(r_{0}^{-2}\right)\right]\right)=\tilde{\eta}
$$

for $\eta$ so that

$$
\eta=\frac{\tilde{\eta}}{1+s_{1}\left[\frac{1}{2}+\mathrm{O}\left(r_{0}^{-2}\right)\right]}+\mathrm{O}_{s_{1}}\left(|\mu \tilde{\eta}|+|\tilde{\eta}|^{3}\right)
$$

and consequently

$$
\left.W_{+}^{\mathrm{s}}(\mu)\right|_{s=s_{1}}: \tilde{u}=\tilde{\eta}\left(\begin{array}{c}
-\frac{s_{1}\left[1+\mathrm{O}\left(r_{0}^{-2}\right)\right]}{2+s_{1}\left[1+\mathrm{O}\left(r_{0}^{-2}\right)\right]} \\
1
\end{array}\right)+\mathrm{O}_{s_{1}}\left(|\mu \tilde{\eta}|+|\tilde{\eta}|^{3}\right)\left(\begin{array}{l}
1 \\
0
\end{array}\right)=\tilde{\eta}\left(\begin{array}{c}
-s_{1} \Gamma_{1} \\
1
\end{array}\right)+\mathrm{O}_{s_{1}}\left(|\mu \tilde{\eta}|+|\tilde{\eta}|^{3}\right)\left(\begin{array}{l}
1 \\
0
\end{array}\right),
$$

where

$$
\Gamma_{1}=\frac{1}{2}\left[1+\mathrm{O}\left(s_{1}+r_{0}^{-2}\right)\right]>0 .
$$

In light of $(5.7), \tilde{u}\left(\tau_{1}\right)$ lies in $W_{+}^{\mathrm{s}}(\mu)$ provided

$$
\tilde{u}_{1}\left(\tau_{1}\right)=-s_{1} \Gamma_{1} \tilde{u}_{2}\left(\tau_{1}\right)+\mathrm{O}_{s_{1}}\left(\left|\mu \tilde{u}_{2}\left(\tau_{1}\right)\right|+\left|\tilde{u}_{2}\left(\tau_{1}\right)\right|^{3}\right)
$$

where $\tau_{1}=\log s_{1}$. Substituting the expressions (5.5) for $\tilde{u}_{j}\left(\tau_{1}\right)$ into (5.9), we obtain the equation

$$
\tilde{u}_{1}^{0}=-\Gamma_{1} s_{1}^{\frac{1}{2}}\left(\left[1+\mathrm{O}\left(s_{1}^{\frac{3}{2}}+r_{0}^{-2}\right)\right] \tilde{u}_{2}^{0}+\mathrm{O}\left(s_{1}^{\frac{3}{2}}\left|\tilde{u}_{1}^{0}\right|\right)\right)+\mathrm{O}_{s_{1}}\left(\mu\left|\tilde{u}^{0}\right|+\sqrt{\mu}\left|\tilde{u}^{0}\right|^{3}\right) .
$$

After choosing $0<s_{1} \ll 1$ sufficiently small, we can solve this equation uniquely for $\tilde{u}_{1}^{0}$ as a function of $\tilde{u}_{2}^{0}$ and get

$$
\tilde{u}_{1}^{0}=-\Gamma_{1} s_{1}^{\frac{1}{2}}\left(1+\mathrm{O}\left(s_{1}^{\frac{3}{2}}+r_{0}^{-2}\right)\right) \tilde{u}_{2}^{0}+\mathrm{O}_{s_{1}}\left(\mu\left|\tilde{u}_{2}^{0}\right|+\sqrt{\mu}\left|\tilde{u}_{2}^{0}\right|^{3}\right) .
$$

We can now substitute $\tilde{u}_{1}^{0}$ into the expressions $(5.6)$ for $\tilde{u}_{j}\left(\tau_{0}\right)$ to get

$$
\begin{aligned}
\tilde{u}_{2}\left(\tau_{0}\right) & =\tilde{u}_{2}^{0} \\
\tilde{u}_{1}\left(\tau_{0}\right) & =\sqrt{\mu} r_{0} s_{1}^{-\frac{1}{2}}\left(\left[1+\mathrm{O}\left(s_{1}^{\frac{3}{2}}+r_{0}^{-2}\right)\right] \tilde{u}_{1}^{0}+\mathrm{O}\left(s_{1}^{\frac{3}{2}}\left|\tilde{u}_{1}^{0}\right|\right)\right) \\
& =\sqrt{\mu} r_{0}\left[-\Gamma_{1}+\mathrm{O}\left(s_{1}^{\frac{3}{2}}+r_{0}^{-2}\right)\right] \tilde{u}_{2}^{0}+\mathrm{O}_{s_{1}}\left(\mu\left|\tilde{u}_{2}^{0}\right|+\sqrt{\mu}\left|\tilde{u}_{2}^{0}\right|^{3}\right)
\end{aligned}
$$

where we used that $\mathrm{e}^{\tau_{0}}=\sqrt{\mu} r_{0}$. With a slight abuse of notation, we shall now set $\tilde{v}_{2}^{0}=\eta$. Using this notation and (5.8), and exploiting (5.4), we find that the stable manifold $W_{+}^{\mathrm{s}}(\mu)$ at $r=r_{0}$ is given by

$$
\left.W_{+}^{\mathrm{s}}(\mu)\right|_{r=r_{0}}: u=\sqrt{\mu} r_{0} \eta\left[-\frac{1}{2}+\mathrm{O}\left(s_{1}+r_{0}^{-2}\right)+\mathrm{O}_{s_{1}}\left(\mu+\sqrt{\mu}|\eta|^{2}\right)\right]\left(\begin{array}{l}
1 \\
0
\end{array}\right)+\eta\left(\begin{array}{l}
0 \\
1
\end{array}\right) .
$$

Next, we use (4.31)-(4.32) to transform this expression to the $(A, B)$-coordinates and obtain

$$
\begin{aligned}
\left.W_{+}^{\mathrm{s}}(\mu)\right|_{r=r_{0}}:\left(\begin{array}{c}
A \\
B
\end{array}\right) & =\sqrt{\mu}\left[\sqrt{\mu} r_{0} \eta\left[-\frac{1}{2}+\mathrm{O}\left(s_{1}+r_{0}^{-2}\right)+\mathrm{O}_{s_{1}}\left(\mu+\sqrt{\mu}|\eta|^{2}\right)\right]\left(\begin{array}{c}
1 \\
\frac{1}{r_{0}}
\end{array}\right)+\eta\left(\begin{array}{c}
1 \\
0
\end{array}\right)\right] \\
& =\sqrt{\mu} \eta\left(\begin{array}{c}
1 \\
0
\end{array}\right)+\mu \eta\left[-\frac{1}{2}+\mathrm{O}\left(s_{1}+r_{0}^{-2}\right)+\mathrm{O}_{s_{1}}\left(\mu+\sqrt{\mu}|\eta|^{2}\right)\right]\left(\begin{array}{c}
r_{0} \\
1
\end{array}\right) .
\end{aligned}
$$


The final step of the analysis consists of finding nontrivial intersections of the stable manifold $W_{+}^{\mathrm{s}}(\mu)$ given above and the core manifold $W_{-}^{\text {cu }}(\mu)$ given by (3.23),

$$
\begin{aligned}
\left.W_{-}^{\mathrm{cu}}(\mu)\right|_{r=r_{0}}:\left(\begin{array}{c}
A \\
B
\end{array}\right)= & \mathrm{e}^{\mathrm{i}\left[-\pi / 4+\mathrm{O}\left(1 / r_{0}^{2}\right)+\mathrm{O}_{r_{0}}\left(|\mu|+|d|^{2}\right)\right]} \times \\
& \times\left(\begin{array}{c}
d_{1}\left[1+\mathrm{O}\left(r_{0}^{-1}\right)\right]-d_{2}\left[\mathrm{i}+\mathrm{O}\left(r_{0}^{-1}\right)\right]+\mathrm{O}_{r_{0}}\left(|\mu||d|+|d|^{2}\right) \\
-d_{2} r_{0}^{-1}\left[\mathrm{i}+\mathrm{O}\left(r_{0}^{-1}\right)\right]-[1 / \sqrt{3}+\mathrm{o}(1)] \sqrt{r_{0}} \nu d_{1}^{2}+\mathrm{O}_{r_{0}}\left(|\mu||d|+\left|d_{2}\right|^{2}+\left|d_{1}\right|^{3}\right)
\end{array}\right) .
\end{aligned}
$$

After redefining $\eta$ to remove the phase in $W_{-}^{\text {cu }}(\mu)$, it remains to solve

$$
\begin{array}{r}
d_{1}-\mathrm{i} d_{2}+\mathrm{O}\left(r_{0}^{-1}\right) d+\mathrm{O}_{r_{0}}\left(\mu|d|+|d|^{2}\right)=\sqrt{\mu} \eta+\mu r_{0} \eta\left[-\frac{1}{2}+\mathrm{O}\left(s_{1}+r_{0}^{-2}\right)+\mathrm{O}_{s_{1}}(\sqrt{\mu})\right] \\
-\frac{\mathrm{i} d_{2}}{r_{0}}+\mathrm{O}\left(r_{0}^{-2}\right) d_{2}-\left[\frac{1}{\sqrt{3}}+\mathrm{o}(1)\right] \sqrt{r_{0}} \nu d_{1}^{2}+\mathrm{O}_{r_{0}}\left(\mu|d|+d_{2}^{2}+d_{1}^{3}\right)=\mu \eta\left[-\frac{1}{2}+\mathrm{O}\left(s_{1}+r_{0}^{-2}\right)+\mathrm{O}_{s_{1}}(\sqrt{\mu})\right] .
\end{array}
$$

If we set $d_{j}=\sqrt{\mu} \hat{d}_{j}$ and write $\eta=\eta_{1}+\mathrm{i} \eta_{2}$, we obtain

$$
\begin{aligned}
\hat{d}_{1}-\mathrm{i} \hat{d}_{2}-\mathrm{i} \eta_{2}+\mathrm{O}\left(r_{0}^{-1}\right) \hat{d}+\mathrm{O}_{r_{0}}\left(\mu|\hat{d}|+\sqrt{\mu}|\hat{d}|^{2}\right) & =\eta_{1}+\sqrt{\mu} r_{0} \mathrm{O}(\eta) \\
-\mathrm{i} \hat{d}_{2}+\mathrm{O}\left(r_{0}^{-1}\right) \hat{d}_{2}-\left[\frac{1}{\sqrt{3}}+\mathrm{o}(1)\right] r_{0}^{\frac{3}{2}} \sqrt{\mu} \nu \hat{d}_{1}^{2}+\mathrm{O}_{r_{0}}\left(\mu|\hat{d}|+\sqrt{\mu} \hat{d}_{2}^{2}+\mu \hat{d}_{1}^{3}\right) & =\sqrt{\mu} r_{0} \eta\left[-\frac{1}{2}+\mathrm{O}\left(s_{1}+r_{0}^{-2}\right)+\mathrm{O}_{s_{1}}(\sqrt{\mu})\right]
\end{aligned}
$$

For $r_{0} \gg 1$ sufficiently large but fixed, we can solve the $(\operatorname{Re} A, \operatorname{Im} A, \operatorname{Im} B)$-components of this equation for $\left(\hat{d}_{1}, \hat{d}_{2}, \eta_{2}\right)$ as a function of $\left(\eta_{1}, \mu\right)$, and we have the expansion

$$
\hat{d}_{1}=\eta_{1}+\mathrm{O}\left(\sqrt{\mu} \eta_{1}\right), \quad \hat{d}_{2}=\mathrm{O}\left(\sqrt{\mu} \eta_{1}\right), \quad \eta_{2}=\mathrm{O}\left(r_{0}^{-1}\right) \eta_{1}+\mathrm{O}\left(\sqrt{\mu} \eta_{1}\right) .
$$

Substituting this solution into (5.11) and projecting it onto the remaining $(\operatorname{Re} B)$-component along the range of the linear map $\hat{d}_{2} \mapsto\left(-\mathrm{i}+\mathrm{O}\left(r_{0}^{-1}\right)\right) \hat{d}_{2}$, we arrive at the equation

$$
-\left[\frac{1}{\sqrt{3}}+\mathrm{o}(1)\right] r_{0}^{\frac{3}{2}} \nu \eta_{1}^{2}+\mathrm{O}_{r_{0}}\left(\mu \eta_{1}\right)=r_{0} \eta_{1}\left[-\frac{1}{2}+\mathrm{O}\left(r_{0}^{-1}\right)+\mathrm{O}\left(s_{1}\right)+\mathrm{O}_{s_{1}}(\sqrt{\mu})\right] .
$$

Factoring out the trivial solution $\eta_{1}=0$, which corresponds to $u=0$, we need to solve

$$
-\left[\frac{1}{\sqrt{3}}+\mathrm{o}(1)\right] \sqrt{r_{0}} \nu \eta_{1}+\mathrm{O}_{r_{0}}(\mu)=-\frac{1}{2}+\mathrm{O}\left(r_{0}^{-1}\right)+\mathrm{O}\left(s_{1}\right)+\mathrm{O}_{s_{1}}(\sqrt{\mu})
$$

which has the unique small solution

$$
\eta_{1}=\frac{\sqrt{3}+\mathrm{O}\left(r_{0}^{-1}\right)+\mathrm{O}\left(s_{1}\right)+\mathrm{O}_{s_{1}}(\sqrt{\mu})}{2 \sqrt{r_{0}} \nu}>0
$$

In particular, using (3.21) to transform back to the original amplitudes $\tilde{d}_{1}$ and $\tilde{d}_{2}$, we see that

$$
\tilde{d}_{1}=\frac{\sqrt{3 \mu}}{2 \nu}>0, \quad \tilde{d}_{2}=\mathrm{O}(\mu)
$$

as claimed. This finishes the proof of Theorem 2.

\section{Planar spot solutions for $\nu \approx 0$}

To prove Theorem 3, we consider the far-field equation (3.7),

$$
\begin{aligned}
& A_{r}=-\frac{\alpha}{2} A+B+\mathcal{R}_{A}(A, B, \alpha, \mu) \\
& B_{r}=-\frac{\alpha}{2} B+\frac{1}{4} \mu A+c_{3}^{0}|A|^{2} A+\mathcal{R}_{B}(A, B, \alpha, \mu),
\end{aligned}
$$


for $(\mu, \nu)$ close to zero, where $\alpha=1 / r$. Applying the scaling

$$
A=\mu^{\frac{1}{4}} a, \quad B=\mu^{\frac{1}{2}} b, \quad r=\mu^{-\frac{1}{4}} s,
$$

we obtain

$$
\begin{aligned}
& a_{s}=b-\frac{a}{2 s}+\mu^{\frac{1}{2}} \mathrm{O}\left(\frac{|a|}{s^{3}}+\frac{|b|}{s^{2}}+(|a|+|b|)^{3}\right) \\
& b_{s}=-\frac{b}{2 s}+\frac{\mu^{\frac{1}{2}} a}{4}+c_{3}^{0}|a|^{2} a+\mu^{\frac{1}{2}} \mathrm{O}\left(\frac{|a|+|b|}{s^{3}}+(|a|+|b|)^{3}\right),
\end{aligned}
$$

where the estimates for the remainder terms are valid for $s \geq s_{1}$ for each fixed $s_{1}>0$. As before, we define

$$
\left(\begin{array}{l}
a \\
b
\end{array}\right)=\frac{1}{\sqrt{s}}\left(\begin{array}{l}
\hat{a} \\
\hat{b}
\end{array}\right)
$$

so that (6.2) becomes

$$
\begin{aligned}
& \hat{a}_{s}=\hat{b}+\mu^{\frac{1}{2}} \mathrm{O}\left(\frac{|\hat{a}|}{s^{3}}+\frac{|\hat{b}|}{s^{2}}+\frac{(|\hat{a}|+|\hat{b}|)^{3}}{s}\right) \\
& \hat{b}_{s}=\frac{\mu^{\frac{1}{2}} \hat{a}}{4}+\frac{c_{3}^{0}}{s}|\hat{a}|^{2} \hat{a}+\mu^{\frac{1}{2}} \mathrm{O}\left(\frac{|\hat{a}|+|\hat{b}|}{s^{3}}+\frac{(|\hat{a}|+|\hat{b}|)^{3}}{s}\right) .
\end{aligned}
$$

Using the scaling

$$
\left(\begin{array}{c}
\hat{a} \\
\hat{b}
\end{array}\right)=\left(\begin{array}{c}
\mu^{\frac{1}{8} \check{a}} \\
\mu^{\frac{3}{8} \check{b}}
\end{array}\right), \quad \rho=\mu^{\frac{1}{4}} s,
$$

we get

$$
\begin{aligned}
& \check{a}_{\rho}=\check{b}+\mu^{\frac{1}{4}} \mathrm{O}\left(\frac{|\check{a}|}{\rho^{3}}+\frac{|\check{b}|}{\rho^{2}}+\mu^{\frac{1}{4}} \frac{(|\check{a}|+|\check{b}|)^{3}}{\rho}\right) \\
& \check{b}_{\rho}=\frac{\check{a}}{4}+\frac{c_{3}^{0}}{\rho}|\check{a}|^{2} \check{a}+\mu^{\frac{1}{4}} \mathrm{O}\left(\frac{|\check{a}|+|\check{b}|}{\rho^{3}}+\mu^{\frac{1}{4}} \frac{(|\check{a}|+|\check{b}|)^{3}}{\rho}\right) .
\end{aligned}
$$

It is now easy to show that, for each fixed $\rho_{1}>0$, there is a $\delta_{1}\left(\rho_{1}\right)>0$ so that the $\rho=\rho_{1}$ fiber of the stable manifold $W_{+}^{\mathrm{s}}(\mu)$ of the solution $(\check{a}, \check{b})=0$ of $(6.5)$ is parametrized by

$$
\left.W_{+}^{\mathrm{s}}(\mu)\right|_{\rho=\rho_{1}}:\left(\begin{array}{c}
\check{a} \\
\check{b}
\end{array}\right)=\tilde{\eta}\left(\begin{array}{c}
1 \\
-\frac{1}{2}
\end{array}\right)+\mathrm{O}\left(\rho_{1}^{-1}|\tilde{\eta}|^{3}+\left|\mu^{\frac{1}{4}}\right||\tilde{\eta}|\right)\left(\begin{array}{c}
1 \\
\frac{1}{2}
\end{array}\right)
$$

where $|\tilde{\eta}|<\delta_{1}\left(\rho_{1}\right)$. To track the stable manifold back to $s=\mu^{\frac{1}{4}} r_{0}$, we use as before the variables

$$
\left(\begin{array}{c}
\tilde{a} \\
\tilde{b}
\end{array}\right)=\left(\begin{array}{c}
a \\
s b-\frac{a}{2}
\end{array}\right)=\frac{1}{\sqrt{s}}\left(\begin{array}{c}
\hat{a} \\
s \hat{b}-\frac{\hat{a}}{2}
\end{array}\right) .
$$

In these coordinates, the stable manifold at $s=s_{1}=\rho_{1} \mu^{-\frac{1}{4}}$ is given by

$$
\begin{aligned}
\left.W_{+}^{\mathrm{s}}(\mu)\right|_{s=s_{1}}:\left(\begin{array}{c}
\tilde{a} \\
\tilde{b}
\end{array}\right) & \stackrel{(6.7)}{=} \frac{1}{\sqrt{s_{1}}}\left(\begin{array}{c}
\hat{a} \\
s_{1} \hat{b}-\frac{\hat{a}}{2}
\end{array}\right) \stackrel{(6.4)}{=} \rho_{1}^{-\frac{1}{2}} \mu^{\frac{1}{8}}\left(\begin{array}{c}
\mu^{\frac{1}{8}} \check{a} \\
\rho_{1} \mu^{-\frac{1}{4}} \mu^{\frac{3}{8}} \breve{b}-\mu^{\frac{1}{8}} \frac{\check{a}}{2}
\end{array}\right) \\
& \stackrel{(6.6)}{=} \rho_{1}^{-\frac{1}{2}} \mu^{\frac{1}{4}} \tilde{\eta}\left(\begin{array}{c}
1+\mathrm{O}\left(\rho_{1}^{-1}|\tilde{\eta}|^{2}+\left|\mu^{\frac{1}{4}}\right|\right) \\
-\frac{1}{2}\left[1+\rho_{1}+\mathrm{O}\left(\rho_{1}^{-1}|\tilde{\eta}|^{2}+\left|\mu^{\frac{1}{4}}\right|\right)\right]
\end{array}\right),
\end{aligned}
$$

where $|\tilde{\eta}|<\delta_{1}\left(\rho_{1}\right)$. Using $\tau=\log s$, equation (6.2) becomes

$$
\begin{aligned}
& \tilde{a}_{\tau}=\tilde{b}+\tilde{\mathcal{R}}_{1}(\tilde{a}, \tilde{b}, s, \mu) \\
& \tilde{b}_{\tau}=\frac{\tilde{a}}{4}+s^{2}\left[\frac{\mu^{\frac{1}{2}} \tilde{a}}{4}+c_{3}^{0}|\tilde{a}|^{2} \tilde{a}\right]+\tilde{\mathcal{R}}_{2}(\tilde{a}, \tilde{b}, s, \mu)
\end{aligned}
$$

in the variables (6.7), and the remainder terms satisfy the following estimates: 
Lemma 7 The remainder terms $\tilde{\mathcal{R}}_{j}(\tilde{a}, \tilde{b}, s, \mu)$ are smooth in $(\tilde{a}, \tilde{b})$ and continuous in $(s, \mu)$ for $s>0$, and they satisfy the estimate

$$
\tilde{\mathcal{R}}_{j}(\tilde{a}, \tilde{b}, s, \mu)=\mu^{\frac{1}{2}} \mathrm{O}\left(s^{-2}\right)(\tilde{a}, \tilde{b})+s \mu^{\frac{1}{2}} \mathrm{O}(|\tilde{a}|+|\tilde{b}|)+\mu^{\frac{1}{2}} \mathrm{O}\left((|\tilde{a}|+|\tilde{b}|)^{3}\right), \quad r_{0} \mu^{\frac{1}{4}} \leq s \leq \rho_{1} \mu^{-\frac{1}{4}}
$$

uniformly in $r_{0} \geq 1$ and $\rho_{1} \leq 1$. This estimate is also valid for the derivatives of $\tilde{\mathcal{R}}_{j}$ with respect to $(\tilde{a}, \tilde{b})$.

We omit the proof of Lemma 7 as it is similar to the proof of Lemma 3. We now proceed as in $\S 5$. Using the variables $u$ from (4.9) and the exponential dichotomies from Lemma 6 together with the associated coordinates $\tilde{u}$, we arrive at the system

$$
\tilde{u}_{\tau}=\left[D+\mathrm{O}\left(\mu^{\frac{1}{2}} \mathrm{e}^{-2 \tau}\right) \mathrm{id}\right] \tilde{u}+c_{3}^{0} \mathrm{e}^{2 \tau}\left|\tilde{u}_{1}+\tilde{u}_{2}\right|^{2}\left(\tilde{u}_{1}+\tilde{u}_{2}\right)\left(\begin{array}{r}
1 \\
-1
\end{array}\right)+\mathrm{O}\left(\mu^{\frac{1}{2}}\right) \mathrm{e}^{2 \tau} \tilde{u}+\mathrm{O}\left(\mu^{\frac{1}{2}}|\tilde{u}|^{3}\right),
$$

where $\tau \in\left[\tau_{0}, \tau_{1}\right]$ with $\mathrm{e}^{\tau_{0}}=s_{0}=r_{0} \mu^{\frac{1}{4}}$ and $\mathrm{e}^{\tau_{1}}=s_{1}=\rho_{1} \mu^{-\frac{1}{4}}$. We convert (6.11) into the integral equation

$$
\begin{aligned}
& \tilde{u}_{1}(\tau)=\mathrm{e}^{\left(\tau-\tau_{1}\right) / 2} \tilde{u}_{1}^{0}+\int_{\tau_{1}}^{\tau} \mathrm{e}^{(\tau-\sigma) / 2}\left(1+\mathrm{O}\left(r_{0}^{-2}\right)\right)\left[\mathrm{O}\left(\mu^{\frac{1}{2}}\right) \mathrm{e}^{2 \sigma} \tilde{u}+\mathrm{O}\left(\left(\mathrm{e}^{2 \sigma}+\mu^{\frac{1}{2}}\right)|\tilde{u}|^{3}\right)\right] \mathrm{d} \sigma \\
& \tilde{u}_{2}(\tau)=\mathrm{e}^{\left(\tau_{0}-\tau\right) / 2} \tilde{u}_{2}^{0}+\int_{\tau_{0}}^{\tau} \mathrm{e}^{(\sigma-\tau) / 2}\left(1+\mathrm{O}\left(r_{0}^{-2}\right)\right)\left[\mathrm{O}\left(\mu^{\frac{1}{2}}\right) \mathrm{e}^{2 \sigma} \tilde{u}+\mathrm{O}\left(\left(\mathrm{e}^{2 \sigma}+\mu^{\frac{1}{2}}\right)|\tilde{u}|^{3}\right)\right] \mathrm{d} \sigma .
\end{aligned}
$$

Next, we set

and obtain

$$
\tilde{u}_{1}=\frac{\mu^{\frac{1}{4}} \check{u}_{1}}{\sqrt{|\log \mu|}}, \quad \tilde{u}_{2}=\frac{\check{u}_{2}}{\sqrt{|\log \mu|}}
$$

$$
\begin{aligned}
\check{u}_{1}(\tau)= & \mathrm{e}^{\left(\tau-\tau_{1}\right) / 2} \check{u}_{1}^{0} \\
& +\int_{\tau_{1}}^{\tau} \mathrm{e}^{(\tau-\sigma) / 2}\left(1+\mathrm{O}\left(r_{0}^{-2}\right)\right)\left[\mathrm{e}^{2 \sigma}\left(\mathrm{O}\left(\mu^{\frac{1}{2}}\right) \check{u}_{1}+\mathrm{O}\left(\mu^{\frac{1}{4}}\right) \check{u}_{2}\right)+\frac{\mathrm{e}^{2 \sigma}+\mu^{\frac{1}{2}}}{|\log \mu|} \mathrm{O}\left(\mu^{\frac{1}{2}}\left|\check{u}_{1}\right|^{3}+\mu^{-\frac{1}{4}}\left|\check{u}_{2}\right|^{3}\right)\right] \mathrm{d} \sigma \\
\check{u}_{2}(\tau)= & \mathrm{e}^{\left(\tau_{0}-\tau\right) / 2} \check{u}_{2}^{0} \\
& +\int_{\tau_{0}}^{\tau} \mathrm{e}^{(\sigma-\tau) / 2}\left(1+\mathrm{O}\left(r_{0}^{-2}\right)\right)\left[\mathrm{e}^{2 \sigma}\left(\mathrm{O}\left(\mu^{\frac{3}{4}}\right) \check{u}_{1}+\mathrm{O}\left(\mu^{\frac{1}{2}}\right) \check{u}_{2}\right)+\frac{\mathrm{e}^{2 \sigma}+\mu^{\frac{1}{2}}}{|\log \mu|} \mathrm{O}\left(\mu^{\frac{3}{4}}\left|\check{u}_{1}\right|^{3}+\left|\check{u}_{2}\right|^{3}\right)\right] \mathrm{d} \sigma .
\end{aligned}
$$

We denote the right-hand sides of (6.13) by $\mathcal{G}_{1}\left(\check{u}^{0}, \check{u}, \mu\right)$ and $\mathcal{G}_{2}\left(\check{u}^{0}, \check{u}, \mu\right)$, respectively. Using the weighted norms

$$
\left\|\check{u}_{1}\right\|_{\mathrm{u}}=\sup _{\tau_{0} \leq \tau \leq \tau_{1}} \mathrm{e}^{-\left(\tau-\tau_{1}\right) / 2}\left|\check{u}_{1}(\tau)\right|, \quad\left\|\check{u}_{2}\right\|_{\mathrm{s}}=\sup _{\tau_{0} \leq \tau \leq \tau_{1}} \mathrm{e}^{-\left(\tau_{0}-\tau\right) / 2}\left|\check{u}_{2}(\tau)\right|,
$$

we find after some algebra that the right-hand sides $\mathcal{G}_{j}\left(\check{u}^{0}, \check{u}, \mu\right)$ of $(6.13)$ satisfy the estimates

$$
\begin{aligned}
\left\|\mathcal{G}_{1}\right\|_{\mathrm{u}} & \leq C\left[\left|\check{u}_{1}^{0}\right|+\rho_{1}^{2}\left\|\check{u}_{1}\right\|+\rho_{1}^{\frac{3}{2}} r_{0}^{\frac{1}{2}}\left\|\check{u}_{2}\right\|+\frac{\rho_{1}^{2}}{|\log \mu|}\left\|\check{u}_{1}\right\|^{3}+\left(\rho_{1}^{\frac{1}{2}} r_{0}^{\frac{3}{2}}+\frac{\rho_{1}^{\frac{1}{2}} r_{0}^{-\frac{1}{2}}}{|\log \mu|}\right)\left\|\check{u}_{2}\right\|^{3}\right] \\
\left\|\mathcal{G}_{2}\right\|_{\mathrm{s}} & \leq C\left[\left|\check{u}_{2}^{0}\right|+\rho_{1}^{\frac{5}{2}} r_{0}^{-\frac{1}{2}}\left\|\check{u}_{1}\right\|+\rho_{1}^{2}\left\|\check{u}_{2}\right\|+\frac{\rho_{1}^{\frac{5}{2}} r_{0}^{-\frac{1}{2}}}{|\log \mu|}\left\|\check{u}_{1}\right\|^{3}+\frac{\rho_{1} r_{0}}{|\log \mu|}\left\|\check{u}_{2}\right\|^{3}\right],
\end{aligned}
$$

where the constant $C$ is independent of $\left(\rho_{1}, r_{0}\right)$ and $\left(\check{u}^{0}, \breve{u}, \mu\right)$. Thus, for any fixed $K>0$ and $r_{0} \geq 1$, there exists a $\check{\rho}_{1}$ such that (6.13) has a unique solution $\check{u}$ for each $\check{u}^{0}$ with $\left|\check{u}^{0}\right| \leq K$ and each $\rho_{1} \leq \check{\rho}_{1}$. This solution also satisfies

$$
\|\check{u}\| \leq C\left|\check{u}^{0}\right|, \quad\left\|\check{u}-\left(\begin{array}{c}
\mathrm{e}^{\left(\cdot-\tau_{1}\right) / 2} \check{u}_{1}^{0} \\
\mathrm{e}^{\left(\tau_{0}-\cdot\right) / 2} \check{u}_{2}^{0}
\end{array}\right)\right\| \leq C \rho_{1}^{\frac{1}{2}}\left|\check{u}^{0}\right| .
$$

We shall see below that $K$ can be chosen as $\sqrt{2 /\left|c_{3}^{0}\right|}$. We now evaluate $\check{u}(\tau)$ at $\tau=\tau_{0}$ and $\tau=\tau_{1}$ and obtain

$$
\begin{aligned}
& \check{u}_{1}\left(\tau_{1}\right)=\check{u}_{1}^{0} \\
& \check{u}_{2}\left(\tau_{1}\right)=\mathrm{e}^{\left(\tau_{0}-\tau_{1}\right) / 2}\left[\check{u}_{2}^{0}+\mathrm{O}\left(\rho_{1}^{2}\left|\check{u}^{0}\right|\right)+\frac{\mathrm{O}\left(\left|\check{u}^{0}\right|^{3}\right)}{|\log \mu|}\right]=\mu^{\frac{1}{4}} r_{0}^{\frac{1}{2}} \rho_{1}^{-\frac{1}{2}}\left[\check{u}_{2}^{0}+\mathrm{O}\left(\rho_{1}^{2}\left|\check{u}^{0}\right|\right)+\frac{\mathrm{O}\left(\left|\check{u}^{0}\right|^{3}\right)}{|\log \mu|}\right]
\end{aligned}
$$


and

$$
\begin{aligned}
& \check{u}_{2}\left(\tau_{0}\right)=\check{u}_{2}^{0} \\
& \check{u}_{1}\left(\tau_{0}\right)=\mathrm{e}^{\left(\tau_{0}-\tau_{1}\right) / 2}\left[\check{u}_{1}^{0}+\mathrm{O}\left(\rho_{1}^{\frac{3}{2}}\left|\check{u}^{0}\right|\right)+\mathrm{O}\left(\rho_{1}^{\frac{1}{2}}\left|\check{u}^{0}\right|^{3}\right)\right]=\mu^{\frac{1}{4}} r_{0}^{\frac{1}{2}} \rho_{1}^{-\frac{1}{2}}\left[\check{u}_{1}^{0}+\mathrm{O}\left(\rho_{1}^{\frac{3}{2}}\left|\check{u}^{0}\right|\right)+\mathrm{O}\left(\rho_{1}^{\frac{1}{2}}\left|\check{u}^{0}\right|^{3}\right)\right] .
\end{aligned}
$$

Setting

$$
\tilde{u}^{0}=\frac{\check{u}^{0}}{\sqrt{|\log \mu|}}, \quad \text { valid for } \quad\left|\tilde{u}^{0}\right| \leq \frac{K}{\sqrt{|\log \mu|}}
$$

and transforming the expressions above back to the $\tilde{u}$-variables using (6.12), we obtain

$$
\begin{aligned}
& \tilde{u}_{1}\left(\tau_{1}\right)=\mu^{\frac{1}{4}} \tilde{u}_{1}^{0} \\
& \tilde{u}_{2}\left(\tau_{1}\right)=\mu^{\frac{1}{4}} r_{0}^{\frac{1}{2}} \rho_{1}^{-\frac{1}{2}}\left[\tilde{u}_{2}^{0}+\mathrm{O}\left(\rho_{1}^{2}\left|\tilde{u}^{0}\right|\right)+\mathrm{O}\left(\left|\tilde{u}^{0}\right|^{3}\right)\right]
\end{aligned}
$$

and

$$
\begin{aligned}
& \tilde{u}_{1}\left(\tau_{0}\right)=\mu^{\frac{1}{2}}\left[r_{0}^{\frac{1}{2}} \rho_{1}^{-\frac{1}{2}} \tilde{u}_{1}^{0}+\mathrm{O}\left(r_{0}^{\frac{1}{2}} \rho_{1}\left|\tilde{u}^{0}\right|\right)-\frac{c_{3}^{0} r_{0}^{2}}{2}|\log \mu|\left|\tilde{u}_{2}^{0}\right|^{2} \tilde{u}_{2}^{0}\left(1+\mathrm{O}\left(\rho_{1}^{\frac{1}{2}}+r_{0}^{-2}\right)\right)+\mathrm{O}_{r_{0}}\left(\left|\tilde{u}^{0}\right|^{3}\right)\right] \\
& \tilde{u}_{2}\left(\tau_{0}\right)=\tilde{u}_{2}^{0} .
\end{aligned}
$$

Indeed, using (6.13) and (6.15), the integral containing the $\mathrm{e}^{2 \tau} c_{3}^{0}|a|^{2} a$-term in the expression for $\check{u}_{1}\left(\tau_{0}\right)$ can be calculated as follows:

$$
\begin{aligned}
\frac{1}{|\log \mu|} \int_{\tau_{1}}^{\tau_{0}} \mathrm{e}^{\left(\tau_{0}-\sigma\right) / 2} \mathrm{e}^{2 \sigma} c_{3}^{0}\left[\mu^{\frac{1}{2}} \mathrm{e}^{3\left(\sigma-\tau_{1}\right) / 2} \mathrm{O}\left(\left|\check{u}^{0}\right|^{3}\right)+\mathrm{e}^{\left(\sigma-\tau_{1}\right) / 2} \mathrm{e}^{\tau_{0}-\sigma} \mathrm{O}\left(\left|\check{u}^{0}\right|^{3}\right)+\mu^{-\frac{1}{4}} \mathrm{e}^{3\left(\tau_{0}-\sigma\right) / 2}\left|\check{u}_{2}^{0}\right|^{2} \check{u}_{2}^{0}\right]\left(1+\mathrm{O}\left(r_{0}^{-2}\right)\right) \mathrm{d} \sigma \\
\quad=\frac{c_{3}^{0}}{|\log \mu|} \mathrm{e}^{\tau_{0} / 2} \int_{\tau_{1}}^{\tau_{0}}\left[\mathrm{O}\left(\left|\check{u}^{0}\right|^{3}\right)\left(\mu^{\frac{1}{2}} \mathrm{e}^{-3 \tau_{1} / 2} \mathrm{e}^{3 \sigma}+\mathrm{e}^{-\tau_{1} / 2} \mathrm{e}^{\tau_{0}} \mathrm{e}^{\sigma}\right)+\mu^{-\frac{1}{4}} \mathrm{e}^{3 \tau_{0} / 2}\left|\check{u}_{2}^{0}\right|^{2} \check{u}_{2}^{0}\right]\left(1+\mathrm{O}\left(r_{0}^{-2}\right)\right) \mathrm{d} \sigma \\
\quad=\frac{c_{3}^{0}}{|\log \mu|} \mathrm{e}^{\tau_{0} / 2}\left[\mathrm{O}\left(\left|\check{u}^{0}\right|^{3}\right)\left(\mu^{\frac{1}{2}} \mathrm{e}^{3 \tau_{1} / 2}+\mathrm{e}^{\tau_{1} / 2} \mathrm{e}^{\tau_{0}}\right)+\mu^{-\frac{1}{4}}\left(\tau_{0}-\tau_{1}\right) \mathrm{e}^{3 \tau_{0} / 2}\left|\check{u}_{2}^{0}\right|^{2} \check{u}_{2}^{0}\right]\left(1+\mathrm{O}\left(r_{0}^{-2}\right)\right) \\
\quad=\frac{-c_{3}^{0} r_{0}^{2}}{2} \mu^{\frac{1}{4}}\left|\check{u}_{2}^{0}\right|^{2} \check{u}_{2}^{0}\left(1+\mathrm{O}\left(\rho_{1}^{\frac{1}{2}}+r_{0}^{-2}\right)\right)+\mathrm{O}_{r_{0}}\left(\frac{\mu^{\frac{1}{4}}\left|\check{u}^{0}\right|^{3}}{|\log \mu|}\right) .
\end{aligned}
$$

It is this resonance between the cubic and linear terms that creates the logarithmic terms in our expansions.

Now that we solved the underlying differential equation in the region $\left[\tau_{0}, \tau_{1}\right]$, we can track the stable manifold $W_{+}^{\mathrm{s}}(\mu)$ from $\tau=\tau_{1}$ to $\tau=\tau_{0}$. Recall from (6.8) that

$$
\left.W_{+}^{\mathrm{s}}(\mu)\right|_{s=s_{1}}: \quad\left(\begin{array}{c}
\tilde{a} \\
\tilde{b}
\end{array}\right)=\frac{\mu^{\frac{1}{4}} \tilde{\eta}}{\rho_{1}^{\frac{1}{2}}}\left(\begin{array}{c}
1+\mathrm{O}\left(\rho_{1}^{-1}|\tilde{\eta}|^{2}+\left|\mu^{\frac{1}{4}}\right|\right) \\
-\frac{1}{2}\left[1+\rho_{1}+\mathrm{O}\left(\rho_{1}^{-1}|\tilde{\eta}|^{2}+\left|\mu^{\frac{1}{4}}\right|\right)\right]
\end{array}\right),
$$

where $|\tilde{\eta}|<\delta_{1}\left(\rho_{1}\right)$. Using the coordinate transformation (4.9) to the $u$-variables and subsequently the coordinate change (5.4) to the exponential-dichotomy variables $\tilde{u}$, we arrive at

$$
\left.W_{+}^{\mathrm{s}}(\mu)\right|_{\tau=\tau_{1}}: \quad \tilde{u}\left(\tau_{1}\right)=\frac{\mu^{\frac{1}{4}} \tilde{\eta}}{\rho_{1}^{\frac{1}{2}}}\left(\begin{array}{c}
-\frac{\rho_{1}}{2}+\mathrm{O}\left(\rho_{1}^{-1}|\tilde{\eta}|^{2}+\left|\mu^{\frac{1}{4}}\right|\right) \\
{\left[1+\frac{\rho_{1}}{2}+\mathrm{O}\left(\rho_{1}^{-1}|\tilde{\eta}|^{2}+\left|\mu^{\frac{1}{4}}\right|\right)\right]\left(1+\mathrm{O}\left(r_{0}^{-2}\right)\right)}
\end{array}\right) .
$$

We need to find $\tilde{u}^{0}$ with $\left|\tilde{u}^{0}\right| \leq K|\log \mu|^{-\frac{1}{2}}$ so that $\tilde{u}\left(\tau_{1}\right)$, as given by (6.16), lies in $W_{+}^{\text {s }}(\mu)$, which leads to the system

$$
\begin{aligned}
\tilde{u}_{1}^{0} & =\tilde{\eta}\left[-\frac{\rho_{1}^{\frac{1}{2}}}{2}+\mathrm{O}\left(\rho_{1}^{-\frac{3}{2}}|\tilde{\eta}|^{2}+\left|\mu^{\frac{1}{4}}\right|\right)\right] \\
r_{0}^{\frac{1}{2}}\left[\tilde{u}_{2}^{0}+\mathrm{O}\left(\rho_{1}^{2}\left|\tilde{u}^{0}\right|\right)+\mathrm{O}\left(\left|\tilde{u}^{0}\right|^{3}\right)\right] & =\tilde{\eta}\left[1+\frac{\rho_{1}}{2}+\mathrm{O}\left(\rho_{1}^{-1}|\tilde{\eta}|^{2}+\left|\mu^{\frac{1}{4}}\right|\right)\right]\left(1+\mathrm{O}\left(r_{0}^{-2}\right)\right)
\end{aligned}
$$

that has the unique solution

$$
\begin{aligned}
\tilde{\eta} & =r_{0}^{\frac{1}{2}}[1+\mathrm{o}(1)]\left(\tilde{u}_{2}^{0}+\mathrm{O}\left(\rho_{1}^{2}\left|\tilde{u}^{0}\right|\right)\right. \\
\tilde{u}_{1}^{0} & =-\frac{\sqrt{r_{0} \rho_{1}}}{2}[1+\mathrm{o}(1)] \tilde{u}_{2}^{0}
\end{aligned}
$$


for $\left|\tilde{u}^{0}\right| \leq K|\log \mu|^{-\frac{1}{2}}$, where o(1) denotes terms that go to zero at $\rho_{1} \rightarrow 0$ and $r_{0} \rightarrow \infty$ uniformly in $\mu$. Substituting (6.18) into (6.17), using (5.4), and setting $\eta:=\tilde{u}_{2}^{0}$, we obtain

$$
\begin{aligned}
& \tilde{u}_{1}\left(\tau_{0}\right)=\mu^{\frac{1}{2}}\left[-\frac{r_{0}}{2}[1+\mathrm{o}(1)] \eta-\frac{c_{3}^{0} r_{0}^{2}}{2}|\log \mu||\eta|^{2} \eta\left[1+\mathrm{o}(1)+\mathrm{O}_{r_{0}}\left(|\log \mu|^{-1}\right)\right]\right] \\
& \tilde{u}_{2}\left(\tau_{0}\right)=\eta
\end{aligned}
$$

for $|\eta| \leq K|\log \mu|^{-\frac{1}{2}}$. Proceeding as in (4.31)-(4.32), except that we use the new scaling (6.1), we arrive at the expansion

$$
\begin{aligned}
\left.W_{+}^{\mathrm{s}}(\mu)\right|_{r=r_{0}}:\left(\begin{array}{c}
A \\
B
\end{array}\right) & =\tilde{u}_{1}\left(\tau_{0}\right)\left(\begin{array}{c}
1 \\
\frac{1}{r_{0}}
\end{array}\right)+\tilde{u}_{2}\left(\tau_{0}\right)\left(\begin{array}{c}
1 \\
0
\end{array}\right) \\
& =\left(\begin{array}{c}
\mu^{\frac{1}{4}} \eta\left[1+\mathrm{O}_{r_{0}}\left(\mu^{\frac{1}{2}}\right)+\mathrm{O}_{r_{0}}\left(\mu^{\frac{1}{2}}|\log \mu||\eta|^{2}\right)\right] \\
\mu^{\frac{3}{4}} \eta\left[-\frac{1}{2}+\mathrm{o}(1)-\frac{c_{3}^{0} r_{0}}{2}|\log \mu||\eta|^{2}\left[1+\mathrm{o}(1)+\mathrm{O}_{r_{0}}\left(|\log \mu|^{-1}\right)\right]\right]
\end{array}\right)
\end{aligned}
$$

in the $(A, B)$-coordinates. On the other hand, (3.23) gives the expansion

$$
\begin{aligned}
\left.W_{-}^{\mathrm{cu}}(\mu)\right|_{r=r_{0}}:\left(\begin{array}{c}
A \\
B
\end{array}\right)= & \mathrm{e}^{\mathrm{i}\left[-\pi / 4+\mathrm{O}\left(1 / r_{0}^{2}\right)+\mathrm{O}_{r_{0}}\left(|\mu|+|d|^{2}\right)\right]} \times \\
& \times\left(\begin{array}{c}
d_{1}\left[1+\mathrm{O}\left(r_{0}^{-1}\right)\right]-d_{2}\left[\mathrm{i}+\mathrm{O}\left(r_{0}^{-1}\right)\right]+\mathrm{O}_{r_{0}}\left(|\mu||d|+|d|^{2}\right) \\
-d_{2} r_{0}^{-1}\left[\mathrm{i}+\mathrm{O}\left(r_{0}^{-1}\right)\right]-[1 / \sqrt{3}+\mathrm{o}(1)] \sqrt{r_{0}} \nu d_{1}^{2}+\mathrm{O}_{r_{0}}\left(|\mu||d|+\left|d_{2}\right|^{2}+\left|d_{1}\right|^{3}\right)
\end{array}\right)
\end{aligned}
$$

for the core manifold. We seek nontrivial intersections of $W_{+}^{\mathrm{s}}(\mu)$ and $W_{-}^{\mathrm{cu}}(\mu)$ at $r=r_{0}$, which, after redefining $\eta$ to remove the phase $\mathrm{e}^{\mathrm{i}[\ldots]}$ in $(6.21)$, can be found as solutions to the system

$$
\begin{aligned}
& d_{1}\left[1+\mathrm{O}\left(r_{0}^{-1}\right)\right]-d_{2}\left[\mathrm{i}+\mathrm{O}\left(r_{0}^{-1}\right)\right]+\mathrm{O}_{r_{0}}\left(|\mu||d|+|d|^{2}\right) \\
& \quad=\mu^{\frac{1}{4}} \eta\left[1+\mathrm{O}_{r_{0}}\left(\mu^{\frac{1}{2}}\right)+\mathrm{O}_{r_{0}}\left(\mu^{\frac{1}{2}}|\log \mu||\eta|^{2}\right)\right] \\
& -d_{2} r_{0}^{-1}\left[\mathrm{i}+\mathrm{O}\left(r_{0}^{-1}\right)\right]-[1 / \sqrt{3}+\mathrm{o}(1)] \sqrt{r_{0}} \nu d_{1}^{2}+\mathrm{O}_{r_{0}}\left(|\mu||d|+\left|d_{2}\right|^{2}+\left|d_{1}\right|^{3}\right) \\
& \quad=\mu^{\frac{3}{4}} \eta\left[-\frac{1}{2}+\mathrm{o}(1)-\frac{c_{3}^{0} r_{0}}{2}|\log \mu||\eta|^{2}\left[1+\mathrm{o}(1)+\mathrm{O}_{r_{0}}\left(|\log \mu|^{-1}\right)\right]\right],
\end{aligned}
$$

where $|\eta| \leq K|\log \mu|^{-\frac{1}{2}}$ for each fixed $K>0$. We can proceed as in $\S 5$ to find nontrivial solutions of (6.22): Writing $\eta=\eta_{1}+\mathrm{i} \eta_{2}$, and solving the $(\operatorname{Re} A, \operatorname{Im} A, \operatorname{Re} B)$-components of $(6.22)$ for $\left(d_{1}, d_{2}, \eta_{2}\right)$ as functions of $\left(\eta_{1}, \mu\right)$, we obtain

$$
d_{1}=\mu^{\frac{1}{4}} \eta_{1}\left[1+\mathrm{O}\left(r_{0}^{-1}\right)\right], \quad\left(d_{2}, \eta_{2}\right)=\frac{\mu^{\frac{1}{4}}}{r_{0}} \mathrm{O}\left(\eta_{1}\right)+\mathrm{O}_{r_{0}}\left(\mu^{\frac{1}{2}}\right) .
$$

Proceeding as in $\S 5$, it remains to solve the equation

$$
-\frac{\nu}{\sqrt{3}}[1+\mathrm{o}(1)] \sqrt{r_{0}} \eta_{1}^{2}+\mathrm{O}\left(\mu^{\frac{1}{2}} \eta_{1}+\mu^{\frac{1}{4}} \eta_{1}^{3}\right)=\mu^{\frac{1}{4}} \eta_{1}\left[-\frac{1}{2}+\mathrm{o}(1)-\frac{c_{3}^{0} r_{0}}{2}|\log \mu| \eta_{1}^{2}[1+\mathrm{o}(1)]\right] .
$$

Dividing by $\eta_{1}$ and using the new variables

$$
\eta_{1}=\frac{\tilde{\eta}_{1}}{\sqrt{r_{0}|\log \mu|}}, \quad \nu=\tilde{\nu} \mu^{\frac{1}{4}} \sqrt{|\log \mu|}
$$

with $\left|\tilde{\eta}_{1}\right| \leq K$, we obtain the equation

$$
1+\mathrm{o}(1)-\frac{2 \tilde{\nu}}{\sqrt{3}}[1+\mathrm{o}(1)] \tilde{\eta}_{1}+\frac{3 \kappa}{4} \tilde{\eta}_{1}^{2}[1+\mathrm{o}(1)]=0 .
$$

where we also substituted $c_{3}^{0}=3 \kappa / 4 \neq 0$. 
First, we set $\nu=0$ and obtain the equation

$$
1+\mathrm{o}(1)+\frac{3 \kappa}{4} \tilde{\eta}_{1}^{2}[1+\mathrm{o}(1)]=0 .
$$

This equation has two real solutions when $\kappa<0$ and none when $\kappa>0$. Thus, for $\kappa<0$, two localized radial spots bifurcate from $\mu=0$, and their amplitudes scale like

$$
\tilde{d}_{1}= \pm \frac{2+\mathrm{o}(1)}{\sqrt{-3 \kappa}} \frac{\mu^{\frac{1}{4}}}{|\log \mu|} \quad \text { amplitude of } J_{0}(r) .
$$

Next, we set $\kappa>0$ and vary $\tilde{\nu}$ near zero. Fold bifurcations of radial spots correspond to double roots $\tilde{\eta}_{1}$ of (6.24). For $\kappa>0$, we find that folds occur when

$$
\tilde{\eta}_{1}= \pm \sqrt{\frac{2}{3 \kappa}}[1+\mathrm{o}(1)], \quad \tilde{\nu}= \pm \frac{3 \sqrt{\kappa}}{2 \sqrt{2}}[1+\mathrm{o}(1)],
$$

where the o(1) term becomes as small as we wish upon making $\rho_{1} \ll 1$ small and $r_{0} \gg 1$ large. In the original variables, we have

$$
\begin{array}{rlrl}
\tilde{d}_{1}= \pm \sqrt{\frac{2}{3 \kappa}}[1+\mathrm{o}(1)] \frac{\mu^{\frac{1}{4}}}{|\log \mu|} & & \text { amplitude of } J_{0}(r) \\
\nu & = \pm \frac{3 \sqrt{\kappa}}{2 \sqrt{2}}[1+\mathrm{o}(1)] \mu^{\frac{1}{4}} \sqrt{|\log \mu|} & & \text { fold curve in parameter space, }
\end{array}
$$

which completes the proof of Theorem 3.

\section{$7 \quad$ Numerical algorithms}

In this section, we briefly outline how we numerically compute localized radial patterns in the Swift-Hohenberg equation and assess their stability with respect to planar perturbations. We also describe how Maxwell curves of one-dimensional roll patterns can be computed numerically.

Initial-value problem solver. To assess the stability of localized radial patterns of the Swift-Hohenberg equation with respect to non-radial perturbations, we use an initial-value problem solver for the planar Swift-Hohenberg equation (1.1). We pose (1.1) on a large square domain with periodic boundary conditions and use the two-dimensional Fourier transform to reduce the resulting nonlinear PDE to an infinite-dimensional system of ordinary differential equations. This system is then truncated at a sufficiently large Fourier mode $N$ and solved in time using the first-order exponential time-stepping algorithm developed in $[9,19]$.

Numerical continuation of localized radial patterns. We compute stationary radial patterns of the planar Swift-Hohenberg equation as solutions of an appropriate boundary-value problem. The radial Swift-Hohenberg equation (1.2) can be written as

$$
u_{r r r r}+\frac{2 u_{r r r}}{r}-\frac{u_{r r}}{r^{2}}+\frac{u_{r}}{r^{3}}+2\left(u_{r r}+\frac{u_{r}}{r}\right)+(1+\mu) u-f(u)=0
$$

where $u=u(r)$ and $r=\sqrt{x^{2}+y^{2}} \in \mathbb{R}^{+}$. To ensure that $u(r)$ corresponds to a smooth solution $u(x, y)$ in cartesian coordinates, we require that $u(r)$ satisfies

$$
u_{r}(0)=0, \quad u_{r r r}(0)=0
$$

at the core $r=0$. We compute solutions of (7.1) on the bounded interval $r \in(0, R)$ for an appropriate large but fixed $R \gg 1$ with Dirichlet boundary conditions

$$
u(R)=0, \quad u_{r r}(R)=0
$$


at $r=R$. Rewriting (7.1)-(7.3) as a first-order system in the variables $\left(u_{1}, u_{2}, u_{3}, u_{4}\right)=\left(u, u_{r}, u_{r r}, u_{r r r}\right)$, we can continue their solutions $u$ in the parameter $\mu$ with $\nu$ fixed or in the parameter $\nu$ with $\mu$ fixed using the boundaryvalue problem solver AUTO2000 [12]. Initial data for continuation are obtained by first solving the one-dimensional Swift-Hohenberg equation and then using parameter continuation to add in the radial terms; see [18, 23].

We remark that we do not need a phase condition since (7.1) is nonautonomous. It is also known that transversely constructed solutions of (1.2) that decay exponentially as $r \rightarrow \infty$ persist as solutions to (7.1)-(7.3) provided $R$ is sufficiently large; see, for instance, [33].

While continuing radial pulses as solutions of (7.1)-(7.3), we can assess their stability with respect to $\mathbb{D}_{k}$-symmetric perturbations for fixed $k \in \mathbb{N}$ by solving simultaneously the linearized partial differential equation restricted to the $k$ th Fourier mode. More precisely, consider the linearization

$$
\mathcal{L} v=(1+\Delta)^{2} v+\left[\mu-f^{\prime}(u(r))\right] v
$$

of the planar Swift-Hohenberg equation (1.1) about a radial pulse $u=u(r)$. Since $\mathcal{L}$ is self-adjoint, it suffices to monitor whether the equation $\mathcal{L} v=0$ has nontrivial solutions to detect bifurcations. We seek such solutions in the form $v(x, y)=\mathrm{e}^{\mathrm{i} k \varphi} w(r)$ for fixed $k \in \mathbb{N}$, where $w(r)$ satisfies the fourth-order differential equation

$$
\left(\partial_{r r}+\frac{1}{r} \partial_{r}-\frac{k^{2}}{r^{2}}\right)^{2} w+\left[1+\mu-f^{\prime}(u)\right] w=0 .
$$

We solve this equation on $(0, R)$ with the boundary conditions

$$
w_{r}(0)=0, \quad w_{r r r}(0)=0, \quad w(R)=0, \quad w_{r r}(R)=0
$$

and the integral condition

$$
\int_{0}^{R} w(r)^{2} \mathrm{~d} r-\gamma=0
$$

for $\gamma \in \mathbb{R}$. In our computations, we focus on perturbations with $\mathbb{D}_{6}$-symmetry and therefore pick $k=6$. We solve (7.1)-(7.3) and (7.4)-(7.6) together in AUTO2000 for $(u, w, \gamma)$ with $(w, \gamma) \equiv(0,0)$ and continue these solutions in $\mu$ for $\nu$ fixed. Bifurcations points corresponding to eigenfunctions with $\mathbb{D}_{6}$-symmetry are then detected in AUTO2000, and we can compute the resulting eigenfunctions in AUTO2000 by branch switching in $\gamma$, where $\mu$ held fixed at the bifurcation value. Subsequently, stability curves can be traced out by fixing $\gamma=1$ and continuing solutions $(u, w, \mu, \nu)$ of $(7.1)-(7.6)$.

Maxwell curves of roll patterns. The Maxwell criterion is a heuristic existence condition for fronts of the onedimensional Swift-Hohenberg equation. It asserts that fronts that connect the trivial state $u=0$ to a spatially periodic roll pattern $u_{\text {per }}(x)$ of period $L$ exist if the roll pattern has vanishing energy and Hamiltonian (see [24] and references therein for a discussion), where the energy is given by

$$
\mathcal{E}(u)=\int_{0}^{L}\left(\frac{1}{2} u_{x x}^{2}-u_{x}^{2}+\frac{1}{2}(1+\mu) u^{2}-F(u)\right) \mathrm{d} x,
$$

where $F^{\prime}=f$ with $F(0)=0$, and the Hamiltonian is defined pointwise for each $x$ by

$$
\mathcal{H}(u)=u_{x} u_{x x x}-\frac{1}{2} u_{x x}^{2}+u_{x}^{2}+\frac{1}{2}(1+\mu) u^{2}-F(u) .
$$

There are various ways for computing Maxwell curves $[5,6,18]$. Here, we consider stationary solutions of the one-dimensional Swift-Hohenberg equation, which satisfy the system

$$
\begin{aligned}
& u_{1}^{\prime}=u_{2} \\
& u_{2}^{\prime}=u_{3} \\
& u_{3}^{\prime}=u_{4} \\
& u_{4}^{\prime}=c u_{2}-2 u_{3}-(1+\mu) u_{1}+F\left(u_{1}\right)
\end{aligned}
$$


on the interval $(0, L)$, where $c$ is a new free parameter. Spatially periodic rolls with zero Hamiltonian satisfy the boundary conditions

$$
\begin{aligned}
& u_{j}(0)=u_{j}(L), \quad j=1, \ldots, 4 \\
& u_{2}(0) u_{4}(0)-\frac{1}{2} u_{3}(0)^{2}+u_{2}(0)^{2}+\frac{1}{2}(1+\mu) u_{1}(0)^{2}-F\left(u_{1}(0)\right)=0 .
\end{aligned}
$$

In addition, we use the integral constraints

$$
\begin{aligned}
\int_{0}^{L}\left(\partial_{x} u_{1}^{*}\right)\left(u_{1}-u_{1}^{*}\right) \mathrm{d} x & =0 \\
\int_{0}^{L}\left(\frac{1}{2} u_{3}^{2}-u_{2}^{2}+\frac{1}{2}(1+\mu) u_{1}^{2}-F\left(u_{1}\right)\right) \mathrm{d} x & =0,
\end{aligned}
$$

where the first condition is the standard phase minimization constraint for periodic solutions ( $u_{1}^{*}$ denotes the periodic solution at a previous continuation step), and the second condition enforces that the energy vanishes along $u_{1}(x)$. The Maxwell curve can now be found by solving system (7.7)-(7.9) in the free parameters $(\mu, \nu, c, L)$.

Implementation. All computations were carried out on PHoenix, a server with two 3GHz dual core Xeon processors and 8GB of RAM running Mac OS 10.4. The initial-value problem solver was implemented in MATLAB. We solved the boundary-value problems described above using AUTO2000 [12]: all AUTO constants except NTST and NTHL were held at their default values. We modified the NTHL array to give the $L^{2}$-norm a weighting of zero in the computation of the pseudo-arclength. Furthermore, we used NTST $=200$ and $R=100$ for all computations except that we chose NTST $=5000$ and $R=1000$ when $\mu$ gets close to zero.

\section{Numerical computations of rings and spots}

In this section, we explore the existence and stability of localized radial solutions of the Swift-Hohenberg equation numerically. In the process, we shall also validate our bifurcation results for radial rings and spots near $\mu=0$. We consider the Swift-Hohenberg equation

$$
u_{t}=-(1+\Delta)^{2} u-\mu u+\nu u^{2}-u^{3}
$$

with a quadratic-cubic nonlinearity and the equation

$$
u_{t}=-(1+\Delta)^{2} u-\mu u+\kappa u^{3}-u^{5}
$$

with a cubic-quintic nonlinearity, where $(x, y) \in \mathbb{R}^{2}$.

\subsection{Planar ring solutions of (8.1)}

We consider localized radial ring solutions of (8.1). For this equation, the coefficient $c_{3}^{0}$ defined in Theorem 1 is given by $c_{3}^{0}=\frac{3}{4}-\frac{19}{18} \nu^{2}$, and we have $c_{3}^{0}<0$ for $\nu>\nu_{*}:=\sqrt{27 / 38} \approx 0.843$. Theorem 1 asserts that, for each fixed $\nu>\nu_{*}$, infinitely many pairs of localized ring solutions bifurcate at $\mu=0$ into the region $\mu>0$. For large $r$, the profiles of the bifurcating ring solutions are given by

$$
u(r) \approx \pm \sqrt{\mu} q_{n}(\sqrt{\mu} r) \cos (r+\varphi),
$$

where the functions $q_{n}(s)$ are homoclinic orbits of the far-field equation (4.5), and the index $n \in \mathbb{N}$ indicates the number of zeros of the envelopes $q_{n}$. We have not been able to find radial rings numerically for $n>0$, as the envelopes $q_{n}$ with $n>0$ correspond to multi-pulse solutions of the far-field equation (4.5) which seem to have very 

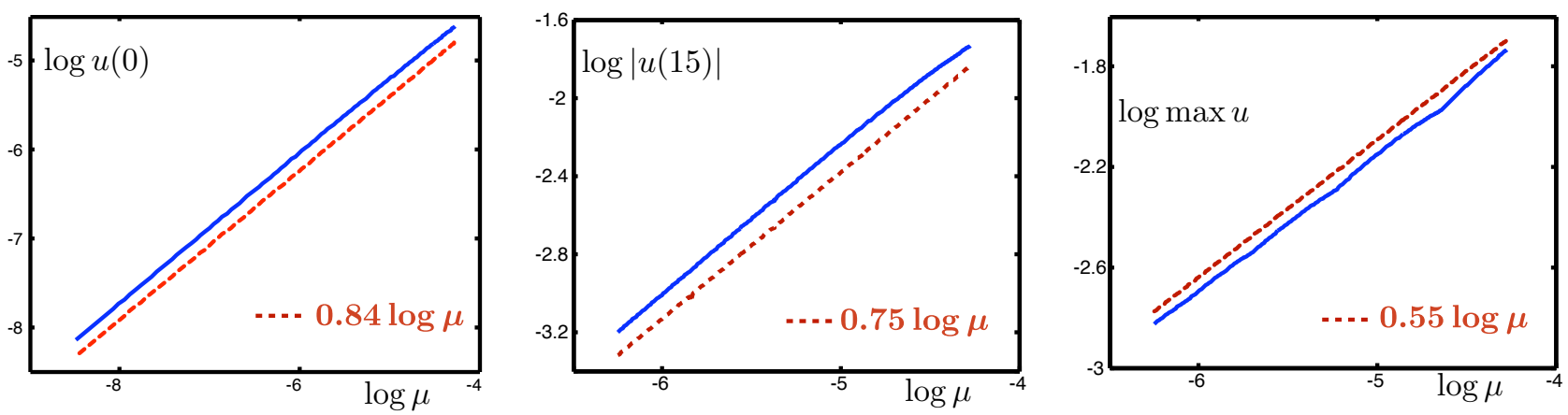

Figure 6: Shown are log-log plots of $u(0), u(15)$ and $\max u$ versus the parameter $\mu$ for localized rings of (8.1) for $\nu=1.6$ with $0<\mu \ll 1$.
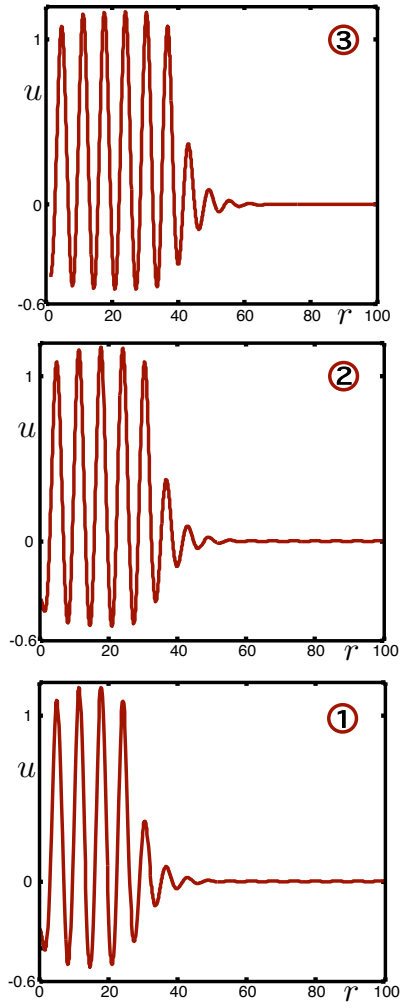
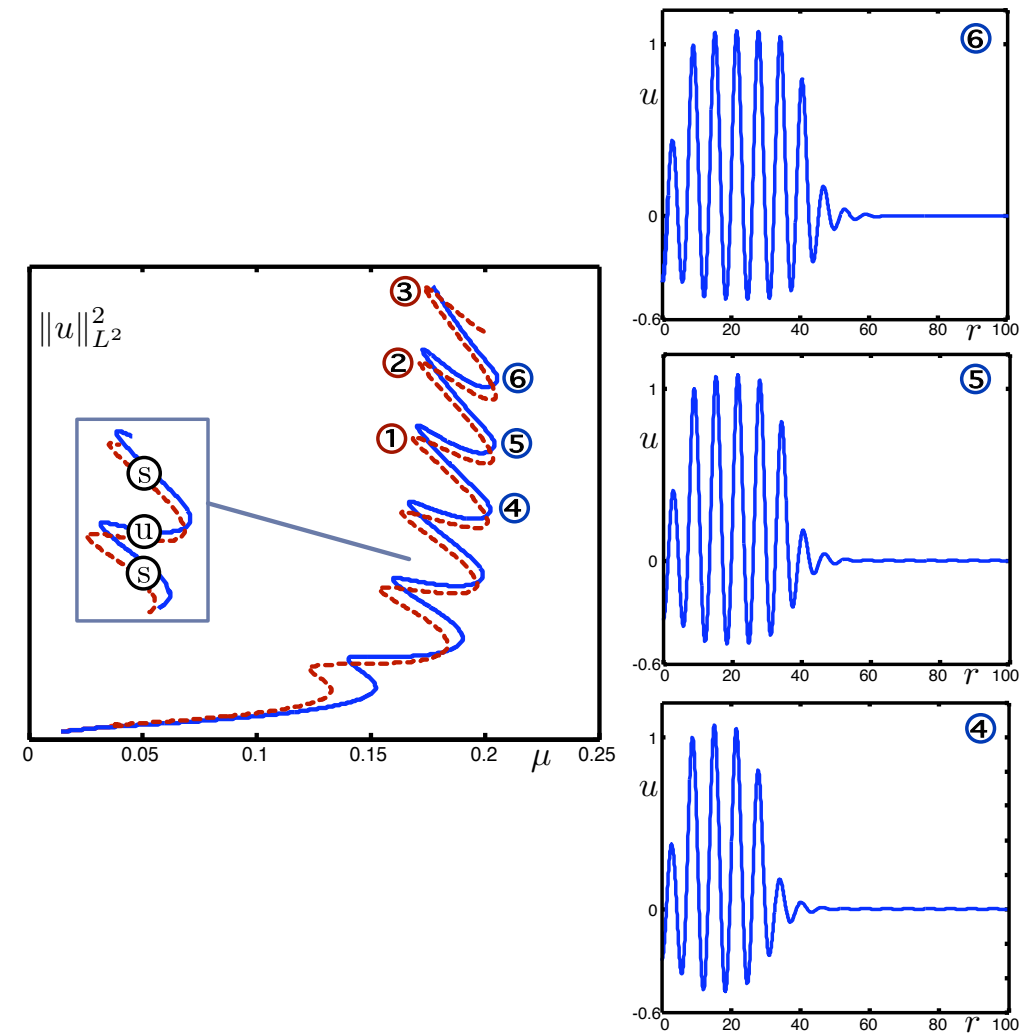

Figure 7: The bifurcation diagram of localized rings of (8.1) with $\nu=1.6$ is shown. The dashed bifurcation curve and the associated solution profiles labelled (1), (2) and (3) correspond to the envelope $-q_{0}$, while the solid bifurcation curve and the associated solution profiles with labels (4), (5) and (6) correspond to the envelope $q_{0}(s)>0$. As one transverses up the solution branches, rolls are added to the ring structures. All rings are unstable with respect to $\mathbb{D}_{6}$-perturbations, but, as indicated in the inset, they are alternately stable (s) and unstable (u) with respect to radial perturbations as one moves up on the solution branches through consecutive folds. 

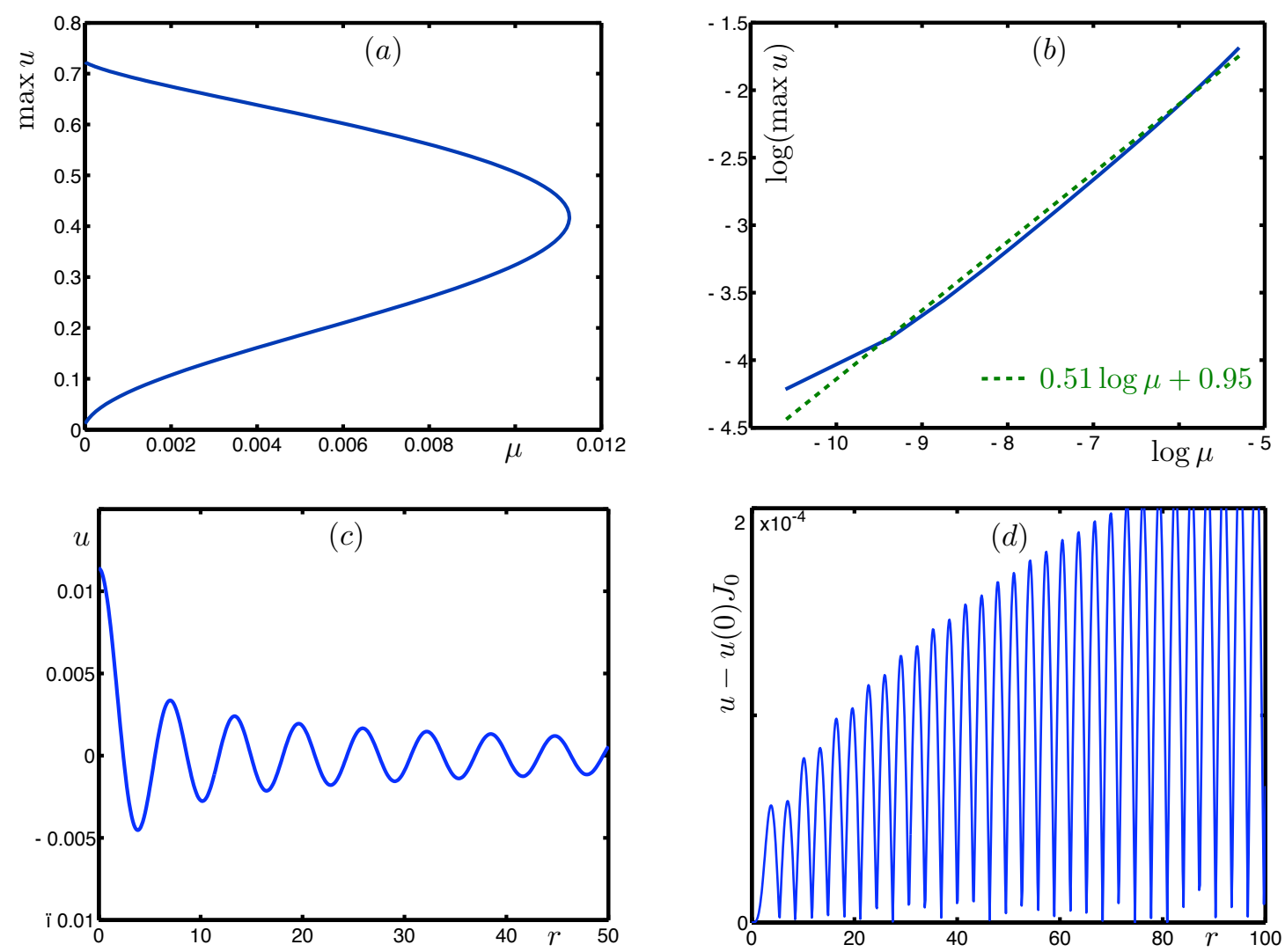

Figure 8: Spots of (8.1) are shown for $\nu=0.8$. (a) Bifurcation diagram of spots. (b) Log-log plot of the maximum height $u(0)$ versus the parameter $\mu$ : the solid line is the result of numerical computations, which we fit with the dashed line $0.51 \log \mu+0.95$. Panels (c) \& (d) contain the profile of a typical spot on the lower branch and its deviation from the Bessel function $J_{0}$.

large separation between consecutive zeros. In the following, we therefore present numerical simulations for the two rings with $n=0$.

First, we check the asymptotic expansions

$$
u(0)=\mathrm{O}\left(\mu^{3 / 4}\right), \quad u\left(r_{0}\right)=\mathrm{O}\left(\mu^{3 / 4}\right), \quad \max _{r \geq 0} u(r)=\mathrm{O}(\sqrt{\mu})
$$

that the analysis in $\S 4$ predicts for $0<\mu \ll 1$ and any fixed $r_{0}>0$. In Figure 6, we plot the values of $u(0), u(15)$ and $\max u(r)$ of numerically computed rings with $n=0$ for $\nu=1.6$ and find very good agreement for these scalings.

Next, we continue the two ring solutions with envelopes $\pm q_{0}(s)$ away from the bifurcation point $\mu=0$. The results are shown in Figure 7. We find that both branches begin to snake between the same well-defined limits: as we move up along each branch, the rings become broader by acquiring more rolls in their interior. Note that the vertical asymptotes of the saddle-node bifurcations on the left and right are close to the snaking limits $\mu=0.181$ and $\mu=0.211$, respectively, of localized rolls in the one-dimensional Swift-Hohenberg equation. This is not surprising as the rings are centered at large values of $r$, where the radial equation is close to the 1D equation. All solutions are unstable to $\mathbb{D}_{6}$-symmetric perturbations but have alternating stability with respect to radial perturbations as indicated in Figure 7.

We remark that, in the 1D case, the different branches of localized rolls are connected by asymmetric localized structures [7], which bifurcate at pitchfork bifurcations from the localized rolls. We do not believe that there is an analogue of this snakes-and-ladder structure in the radial case, since there is no evident mechanism for creating additional bifurcations along the solution branches. 

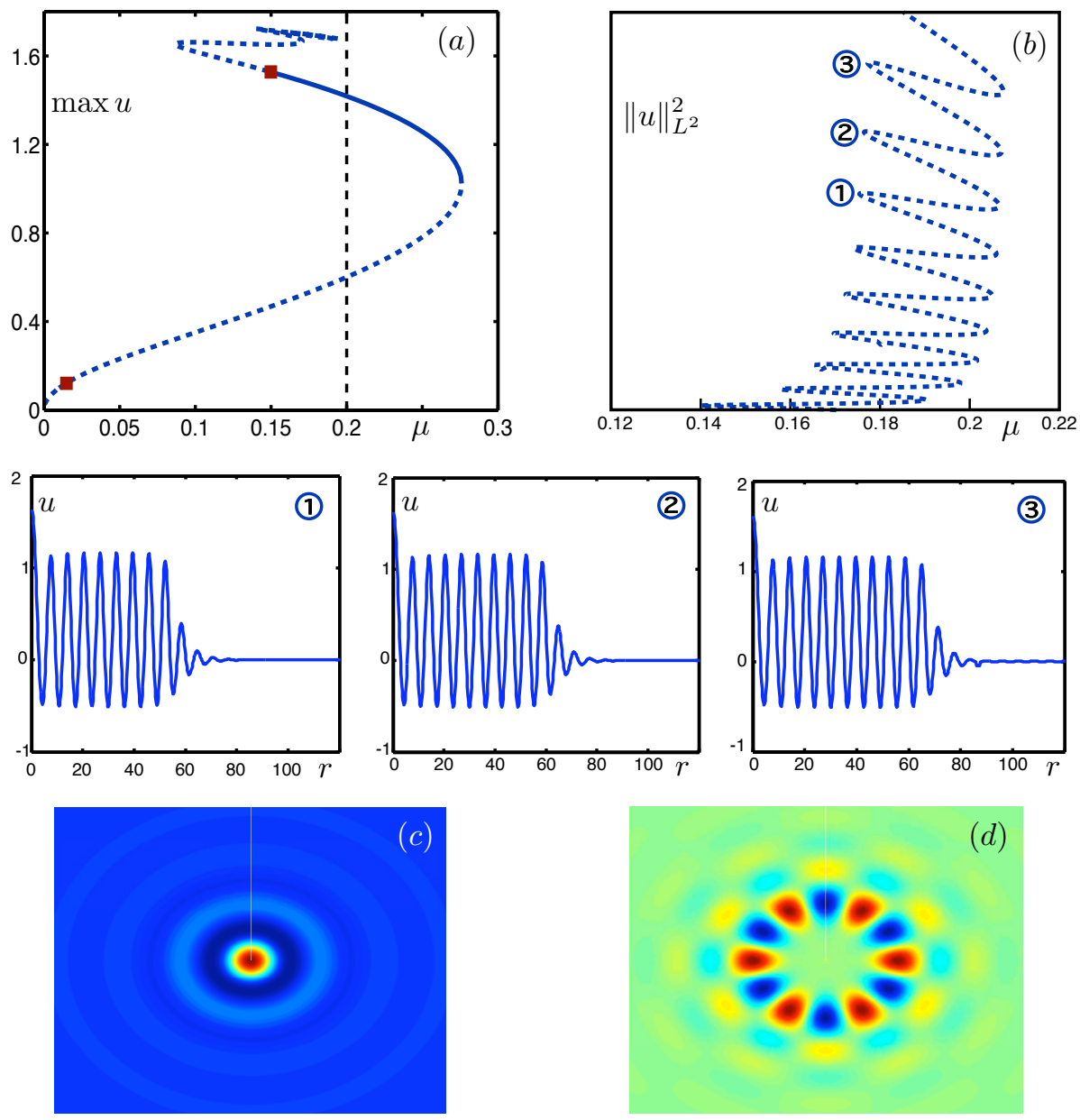

Figure 9: The bifurcation diagram of spots of (8.1) with $\nu=1.6$ is shown in panels (a)-(b). Unstable solutions are indicated by dash lines and stable solutions by solid lines, and the vertical line indicates the Maxwell point of $1 D$ rolls that occurs for $\mu=0.2$. Spots undergo two $\mathbb{D}_{6}$-symmetry breaking bifurcations that are indicated by squares in panel (a). Panels (c) and (d) contain color plots of the spot and the associated neutral localized eigenmode at the second symmetry-breaking bifurcation at $\mu=0.15$.

\subsection{Planar spots of (8.1)}

Theorem 2 asserts the existence of spots of (8.1) for any fixed nonzero $\nu$ when $0<\mu \ll 1$. In particular, spots should exist for $\nu<\nu_{*}=\sqrt{27 / 38} \approx 0.843$, where rings cannot exist. We compute spots numerically for $\nu=0.8<\nu_{*}$ and summarize the results in Figure 8. Spots do bifurcate off $u=0$ at $\mu=0$ and turn around at a saddle-node bifurcation at $\mu=0.0118$. At this fold, spots regain stability with respect to radial perturbations, but they remain unstable with respect to hexagonal perturbations with $\mathbb{D}_{6}$-symmetry. The spot then passes through $\mu=0$ with nonzero amplitude and turns into a non-localized target pattern: this is reminiscent of the Turing instability of $1 \mathrm{D}$ pulses considered in [34]. The computations shown in Figure 8 also confirm the scaling $u(0) \approx \sqrt{\mu}$ as $\mu \rightarrow 0$ that Theorem 2 asserts. Finally, a comparison of the spot profile with the Bessel function $J_{0}(r)$ is shown in Figure $8(\mathrm{~d})$ : we see that the core is approximated well, while the error increases as we move into the far field. The oscillations visible in Figure 8(d) are due to phase shifts in the underlying roll pattern $\cos (r+\varphi)$, which we did not factor in.

Next, we compute spots for $\nu=1.6>\nu_{*}$ and summarize the results in Figure 9. Panel (a) gives the bifurcation diagram of spots: as predicted by the analysis, spots bifurcate off $u=0$ at $\mu=0$. Initially, spots are unstable with respect to radial and hexagonal perturbations. At $\mu=0.015$, spots undergo a $\mathbb{D}_{6}$-symmetry breaking bifurcation: from this point onwards, spots are unstable only with respect to radial perturbations. They gain full PDE stability 


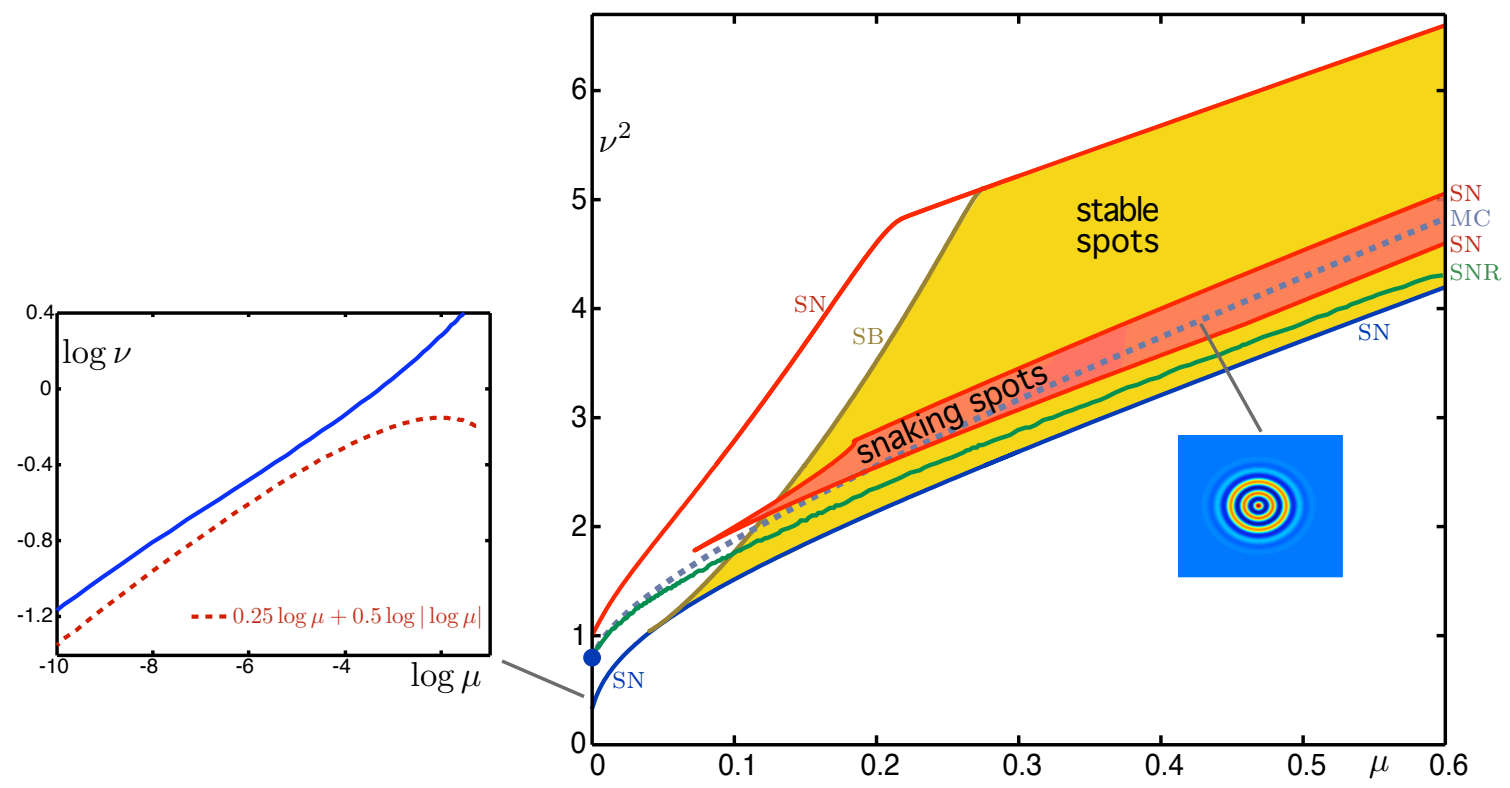

Figure 10: The bifurcation diagram of spots of (8.1) in the $(\mu, \nu)$-parameter space is shown. Folds and symmetrybreaking bifurcations are indicated by the labels $\mathrm{SN}$ and $\mathrm{SB}$, respectively. The Maxwell curve and the fold curve of $1 D$ rolls are labelled $\mathrm{MC}$ and SNR, respectively. Spots are linearly stable inside the light shaded region and snake inside the darker shaded region. The upper fold of spots, the Maxwell curve and the fold curve of rolls all terminate on the $\nu$-axis at $\nu=\sqrt{27 / 38}$, which corresponds to $c_{3}^{0}=0$ and is indicated by the bullet. A log-log plot of the lower fold of spots near the origin is shown in the inset, confirming the scaling predicted in Theorem $3($ ii).

at a fold bifurcation at $\mu=0.27$. At $\mu=0.15$, a second $\mathbb{D}_{6}$-symmetry breaking bifurcation occurs: the spot and the localized neutral eigenmode with $\mathbb{D}_{6}$-symmetry are plotted in Figure $9(\mathrm{c})$-(d). This bifurcation creates localized hexagon patches, which were studied in detail in [24]. The spots then proceed to undergo a sequence of fold bifurcations near the 1D Maxwell point located at $\mu=0.2$. Details of the resulting snaking behaviour are shown in Figure 9(b), where we plot the $L^{2}$-norm of the radial spot profiles against the parameter $\mu$. As one moves up on the branch, rolls are added one by one to the tails of the spots: for the $2 \mathrm{D}$ pattern, this corresponds to adding concentric rings that surround the spot; see Figure 1. Figure 9 shows that the amplitude at the core is still much larger than the amplitude of the concentric rings that are added.

Figure 10 summarizes the existence region of localized spots of the quadratic-cubic Swift-Hohenberg equation (8.1). We find a large parameter region where spots are linearly stable, and a wedge-shaped region where spots snake. Spots lose stability in two different ways: the upper stability boundary corresponds to the $\mathbb{D}_{6}$-symmetry breaking bifurcation, where localized hexagon patches bifurcate from spots. The lower stability boundary is a fold curve: this curve continues all the way to the origin $(\mu, \nu)=0$ and coincides therefore with the fold curve analysed in Theorem 3(ii). The predicted scaling $\nu=\mu^{\frac{1}{4}}|\log \mu|^{\frac{1}{2}}$ is confirmed in the inset by numerical computations. We remark that stable spots exist below the existence region of 1D roll patterns, which is demarcated in Figure 10 by the fold curve of 1D rolls labelled SNR. The bifurcation diagram shown in Figure 10 forms the radial part of the much richer bifurcation diagram for spots and localized hexagon patches described in [24, Figure 33] to which we refer the reader for more details.

\subsection{Planar spots of (8.2)}

Theorem 3(i) asserts that spots exist also in the cubic-quintic Swift-Hohenberg equation (8.2). The predicted amplitude scaling is

$$
u(0) \approx \mu^{\frac{1}{4}}|\log \mu|^{-\frac{1}{2}}
$$




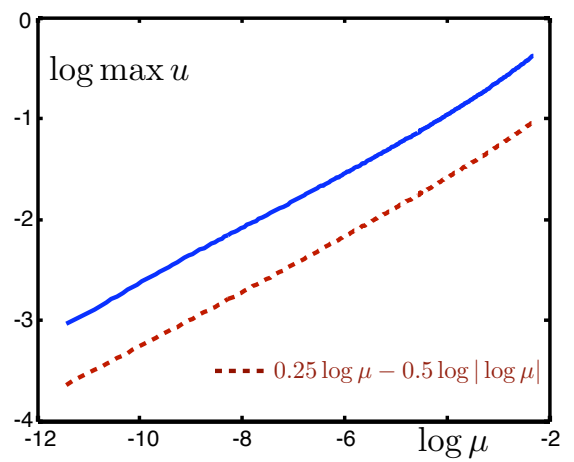

Figure 11: Log-log plot of the amplitude u(0) of spots of the cubic-quintic Swift-Hohenberg equation (8.2) for $\nu=1.6$.

so that spots of (8.2) bifurcate with much larger amplitudes than the spots of (8.1) that have amplitude proportional to $\mu^{\frac{1}{2}}$. Figure 11 confirms the scaling (8.3).

\section{$9 \quad$ Summary and open problems}

In this paper, we proved that localized radial structures bifurcate subcritically from homogeneous rest states that undergo Turing instabilities. Ring-like structures bifurcate when the Turing bifurcation is subcritical, that is, when stripe patterns coexist with the stable homogeneous rest state. In contrast, localized spots are found to exist for arbitrary quadratic nonlinearities irrespective of the criticality of the Turing bifurcation. The major implication is that coexistence of a patterned state and the trivial state is not required for localized spots to exist. This is an intrinsically 2D result, since 1D localized patterns require a subcritical bifurcation for existence, and suggests that other bifurcations should be inspected to see whether they can give rise to localized structures. Our proofs focused on the Swift-Hohenberg equation, with an arbitrary nonlinearity, but our results are also applicable to reaction-diffusion systems due to the center-manifold reduction near Turing bifurcations that was proved for planar radial patterns in [36, Chapter 3].

Our analysis of localized rings required a subcritical Turing bifurcation. In this situation, rings and spots can snake so that a family of localized radial structures with an arbitrary spatial width coexist. Localized rings of vegetation that expand have been observed in the Negev desert, see [26, 37]. Numerically, localized rings tend to be unstable with respect to hexagonal perturbations in the quadratic-cubic Swift-Hohenberg equation (though they can stabilize in certain parameter regions). However, rings might be stable in larger parameter regions for other models.

Localized spots have been found in a variety of physical experiments, for example, in gas discharge systems [3, 4, 15, 27], nonlinear optics [25], chemical reactions [11, 21, 41], and ferro-fluid experiments [32] to name but a few. In most of these systems, there is bistability between Turing patterns and the homogeneous rest state.

One exception are the ferro-fluid experiments of Richter [32], where domain-covering stripes are found to bifurcate supercritically [31]. In these experiments, both domain-covering hexagons and isolated ferro-solitons were found, but localized patches of hexagons were not observed. The height of the radially-symmetric ferro-solitons is far larger than that of the domain-covering hexagons. We will now provide a possible interpretation of this experiment, by analogy with the Swift-Hohenberg equation. In [24], domain-covering hexagons were found to be bistable with the trivial state and to exist in a large open parameter region in the regime where the Turing bifurcation is supercritical. However, localized hexagon patches were found to exist only in a very small wedge in parameter space. Furthermore, these patches have large spatial extent. In the ferro-fluid experiments, the dish is comparatively small compared to the ferro-soliton, and we therefore speculate that a very large dish would be required in order to observe localized hexagon patches in this experiment. The observed ferro-solitons correspond to the spots that we found in the Swift-Hohenberg equation with one key difference: in the ferro-fluid experiment, spots are stable unlike in the Swift- 

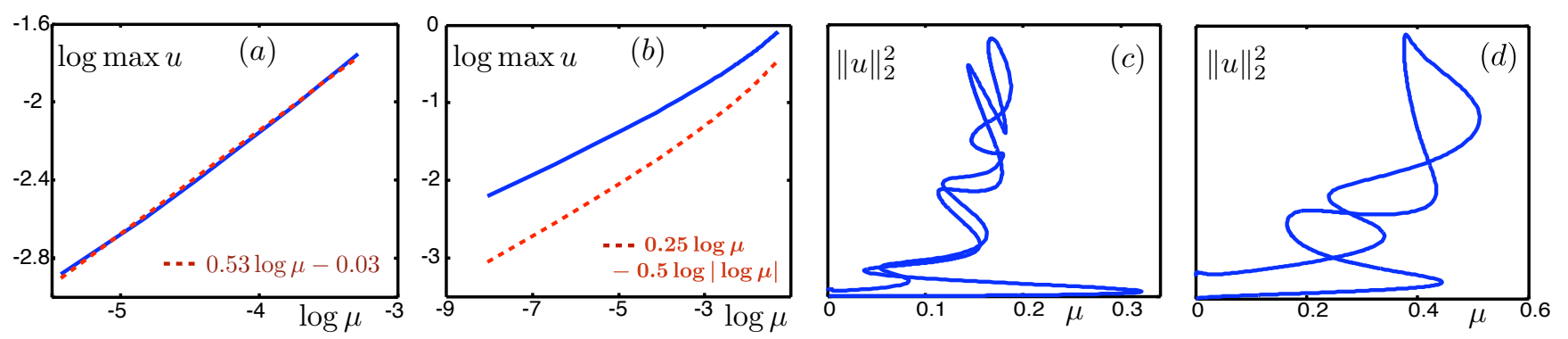

Figure 12: Results for radial spots in three space dimensions are shown. Panels (a) and (b) contain log-log plots of the amplitude $\max u$ of $3 D$ spots versus the parameter $\mu$ respectively for the quadratic-cubic Swift-Hohenberg equation (8.1) with $\nu=1.6$ and the cubic-quintic equation (8.2) with $\nu=2$. The associated bifurcation diagrams away from onset are shown in panel (c) for equation (8.1) and in panel (d) for equation (8.2).

Hohenberg equation. As in the supercritical regime of the Swift-Hohenberg equation, ferro-solitons eventually cross into the region where the spatially homogeneous state loses temporal stability. In particular, these spots do not snake. In order to observe the effects of homoclinic snaking in the ferro-fluid experiment, one would need a fluid that allows for stripes to bifurcate subcritically.

Finally, we outline open problems. One interesting issue is the existence of radial spots in three space dimensions. Such solutions are of particular interest in nonlinear optics, where they correspond to light bullets. In Figure 12, we show numerical computations of the quadratic-cubic and the cubic-quintic Swift-Hohenberg equation which indicate that radial 3D spots bifurcate in the same fashion and with identical scaling as the planar spots discussed in Theorems 2 and 3. We believe that the bifurcation analysis presented in this paper can be extended to the 3D case. Interestingly, Figure 12(c)-(d) indicates that 3D spots do not appear to snake as we continue these solutions away from the bifurcation point at $\mu=0$ : we do not currently understand this behavior.

In Theorem 3(ii), we proved the existence of localized radial spots near $(\mu, \nu)=0$. The computations shown in Figure 8(a) indicate that these spots turn into non-localized stationary target patterns when their branch crosses into the region $\mu<0$ after it turned back at the fold bifurcation. It would be interesting to study the transition to target patterns analytically as these structures have their maximal amplitude at the core, in contrast to the ring-like target patterns found in [36].

One issue that we did not address from a theoretical viewpoint is the stability of spots and rings. We believe that linear stability will depend on the specific form of the nonlinearity: it would be interesting to see whether the small-amplitude spots of the Swift-Hohenberg equation are indeed unstable with respect to only radial and hexagonal perturbations. It is worthwhile to recall that ferro-solitons can be stable, which indicates that there may be circumstances where small-amplitude spots are unstable only to radial perturbations.

The tails of the localized spots we found decay in an oscillatory fashion to zero as $r \rightarrow \infty$. This suggests that bound states of several spots may exist, and certain bound-state configurations have indeed been analysed in [42]. It would be interesting to study bound states more systematically using, for instance, the theory developed recently in [45].

A very interesting class of solutions are time-periodic localized radial structures, which are often referred to as oscillons. Such solutions have been observed in many different experiments, often under subharmonic forcing, and we refer the reader to $[2,22,38,40]$ for examples. In one space dimension, bifurcations to oscillons have been studied recently in [44] near spatially homogeneous Hopf bifurcations with subharmonic forcing. In [36], the bifurcation to target patterns at supercritical Hopf bifurcations was analysed. It would be interesting to see whether radial spots bifurcate near subcritical Hopf bifurcations, possibly under temporal forcing.

Acknowledgements. We thank the Newton Institute for the hospitality during the theme programme Pattern Formation in Large Domains in Autumn 2005. Björn Sandstede gratefully acknowledges a Royal Society Wolfson 
Research Merit Award.

\section{References}

[1] M. Abramowitz and I. A. Stegun. Handbook of Mathematical Functions with Formulas, Graphs, and Mathematical Tables. Dover, New York, 1972.

[2] H. Arbell and J. Fineberg. Temporally harmonic oscillons in Newtonian fluids. Phys. Rev. Lett. 85 (2000) $756-759$.

[3] Y. A. Astrov and H. G. Purwins. Plasma spots in a gas discharge system: birth, scattering and formation of molecules. Phys. Lett. A 283 (2001) 349-354.

[4] Y. A. Astrov and H. G. Purwins. Spontaneous division of dissipative solitons in a planar gas-discharge system with high ohmic electrode. Phys. Lett. A 358 (2006) 404-408.

[5] C. J. Budd, G. W. Hunt and R. Kuske. Asymptotics of cellular buckling close to the Maxwell load. R. Soc. Lond. Proc. Ser. A 457 (2001) 2935-2964.

[6] C. J. Budd and R. Kuske. Localized periodic patterns for the non-symmetric generalized Swift-Hohenberg equation. Phys. D 208 (2005) 73-95.

[7] J. Burke and E. Knobloch. Localized states in the generalized Swift-Hohenberg equation. Phys. Rev. E 73 (2006) 056211.

[8] J. Burke and E. Knobloch. Normal form for spatial dynamics in the Swift-Hohenberg equation. Discr. Cont. Dyn. Sys. Suppl. (2007) 170-180. (September issue).

[9] S. M. Cox and P. C. Matthews. Exponential time differencing for stiff systems. J. Comput. Phys. 176 (2002) $430-455$.

[10] M. Cross and P. Hohenberg. Pattern formation outside of equilibrium. Rev. Mod. Phys. 65 (1993) 851-1112.

[11] P. Davies, P. Blanchedeau, E. Dulos and P. D. Kepper. Dividing blobs, chemical flowers and patterned islands in a reaction-diffusion system. J. Phys. Chem. A 102 (1998) 8236-8244.

[12] E. J. Doedel, R. C. Paffenroth, A. R. Champneys, T. F. Fairgrieve, Y. A. Kuznetsov, B. E. Oldeman, B. Sandstede and X. J. Wang. AUTO2000: Continuation and bifurcation software for ordinary differential equations. Technical report, Concordia University, 2000. Available via http://www.dynamicalsystems.org/.

[13] C. Elphick, E. Tirapegui, M. E. Brachet, P. Coullet and G. Iooss. A simple global characterization for normal forms of singular vector fields. Phys. D 29 (1987) 95-127.

[14] P. C. Fife. Pattern formation in gradient systems. In: Handbook of Dynamical Systems 2 (B. Fiedler, ed.). North-Holland, Amsterdam, 2002, 679-719.

[15] W. J. Firth, L. Columbo and T. Maggipinto. On homoclinic snaking in optical systems. Chaos 17 (2007) 037115.

[16] M. D. Groves. Solitary-wave solutions to a class of fifth-order model equations. Nonlinearity 11 (1998) 341-353.

[17] R. B. Hoyle. Pattern Formation. Cambridge University Press, Cambridge, 2006.

[18] G. W. Hunt, M. A. Peletier, A. R. Champneys, P. D. Woods, M. A. Wadee, C. J. Budd and G. J. Lord. Cellular buckling in long structures. Nonlin. Dynam. 21 (2000) 3-29.

[19] A.-K. Kassam and L. N. Trefethen. Fourth-order time-stepping for stiff PDEs. SIAM J. Sci. Comput. 26 (2005) $1214-1233$.

[20] I. Kuzin and S. Pohozaev. Entire Solutions of Semilinear Elliptic Equations. Birkhäuser Verlag, Basel, 1997.

[21] K. J. Lee, W. D. McCormick, J. E. Pearson and H. L. Swinney. Experimental observation of self-replicating spots in a reaction diffusion system. Nature 369 (1994) 215-218. 
[22] O. Lioubashevski, Y. Hamiel, A. Agnon, Z. Reches and J. Fineberg. Oscillons and propagating solitary waves in vertically vibrated colloidal suspension. Phys. Rev. Lett. 83 (1999) 3190-3193.

[23] D. J. B. Lloyd and A. R. Champneys. Efficient numerical continuation and stability analysis of spatiotemporal quadratic optical solitons. SIAM J. Sci. Comput. 27 (2005) 759-773.

[24] D. J. B. Lloyd, B. Sandstede, D. Avitabile and A. R. Champneys. Localized hexagon patterns of the planar SwiftHohenberg equation. SIAM J. Appl. Dynam. Syst. 7 (2008) 1049-1100.

[25] Y. Menesguen, S. Barbay, X. Hachair, L. Leroy, I. Sagnes and R. Kuszelewicz. Optical self-organization and cavity solitons in optically pumped semiconductor microresonators. Phys. Rev. A $\mathbf{7 4}$ (2006) 023818.

[26] E. Meron, H. Yizhaq and E. Gilad. Localized structures in dryland vegetation: forms and functions. Chaos 17 (2007) 037109.

[27] S. Nasuno. Dancing "atoms" and "molecules" of luminous gas-discharge spots. Chaos 13 (2003) 1010-1013.

[28] L. A. Peletier and W. C. Troy. Spatial Patterns. Birkhäuser Verlag, Boston, 2001.

[29] L. M. Pismen. Patterns and Interfaces in Dissipative Dynamics. Springer Verlag, Berlin, 2006.

[30] M. I. Rabinovich, A. B. Ezersky and P. D. Weidman. The Dynamics of Patterns. World Scientific Publishing, River Edge, 2000 .

[31] R. Richter. Private communication (2008).

[32] R. Richter and I. V. Barashenkov. Two-dimensional solitons on the surface of magnetic fluids. Phys. Rev. Lett. 94 (2005) 184503.

[33] B. Sandstede. Convergence estimates for the numerical approximation of homoclinic solutions. IMA J. Numer. Anal. 17 (1997) 437-462.

[34] B. Sandstede and A. Scheel. Absolute instabilities of standing pulses. Nonlinearity 18 (2005) 331-378.

[35] A. Scheel. Subcritical bifurcation to infinitely many rotating waves. J. Math. Anal. Appl. 215 (1997) $252-261$.

[36] A. Scheel. Radially symmetric patterns of reaction-diffusion systems. Mem. Amer. Math. Soc. 165 (2003).

[37] E. Sheffer, H. Yizhaq, E. Gilad, M. Shachak and E. Meron. Why do plants in resource-derived environments form rings? Ecol. Complexity 4 (2007) 192-200.

[38] L. Stenflo and M. Yu. Oscillons at a plasma surface. Phys. Plasmas 10 (2003) 912-913.

[39] J. Swift and P. C. Hohenberg. Hydrodynamic fluctuations at the convective instability. Phys. Rev. A 15 (1977) $319-328$.

[40] P. B. Umbanhowar, F. Melo and H. L. Swinney. Localized excitations in a vertically vibrated granular layer. Nature 382 (1996) 793-796.

[41] V. K. Vanag and I. R. Epstein. Localised patterns in reaction-diffusion systems. Chaos 17 (2007) 037110.

[42] A. G. Vladimirov, J. M. McSloy, D. V. Skryabin and W. J. Firth. Two-dimensional clusters of solitary structures in driven optical cavities. Phys. Rev. E 65 (2002) 046606.

[43] G. N. Watson. A Treatise on the Theory of Bessel Functions. Cambridge University Press, Cambridge, 1944.

[44] A. Yochelis, J. Burke and E. Knobloch. Reciprocal oscillons and nonmonotonic fronts in forced nonequilibrium systems. Phys. Rev. Lett. 97 (2006) 254501.

[45] S. Zelik and A. Mielke. Weak interaction in multi-pulse structures and space-time chaos in reaction-diffusion equations. Mem. Amer. Math. Soc. (accepted). 